\title{
TOPOLOGY OF THE CAUSAL BOUNDARY FOR STANDARD STATIC SPACETIMES
}

\author{
José L. Flores and Steven G. Harris
}

\section{Introduction and Chief Results}

The causal boundary of a strongly causal spacetime, introduced by Geroch, Kronheimer, and Penrose in [GKP], is a tool for examining the causal nature of a spacetime "at infinity". As it is conformally invariant, it is a property of the conformal structure of the spacetime. The addition of the causal boundary to a spacetime results in an object whose structure might be most naturally characterized as a "chronological set", i.e., a set with a chronology relation, a relation extending that on the spacetime. (In a spacetime, $p$ is said to chronologically precede $q$, or $p \ll q$, if there is a future-directed timelike curve from $p$ to $q$. There is also the causality relation: $p$ causally precedes $q$, or $p \prec q$, if there is a futuredirected causal curve from $p$ to $q$.) What is desired is a topology on this object so that the causal boundary puts appropriate endpoints on timelike curves which are endless in the spacetime; but there is a deal of controversy on how this might best be done. The topology suggested in [GKP] has some severe problems, most notable of which is that in the simplest of all cases, Minkowski $n$-space $\mathbb{L}^{n}$, it fails to give what most consider the obvious topology for the spacetime-cum-boundary, that obtained from the conformal embedding of $\mathbb{L}^{n}$ into the Einstein static space, $\mathbb{L}^{1} \times \mathbb{S}^{n-1}$ (see $[\mathrm{HE}]$ ). Some attempts have been made to ameliorate the general problems with this topology (such as $[\mathrm{BS}]$ and $[\mathrm{S}]$ ), but they have no effect on the issue with Minkowski space. A new approach can be found in [MR], suggesting a substantial modification of the GKP method in a somewhat complex but also elegant manner; a related approach that also combines methods used here is to be found in $[\mathrm{F}]$.

None the less, the basic ideas in the causal boundary have much to recommend them; in particular, the separate elements of the future and past causal boundaries - that portion of the GKP construction designed to place future endpoints on future-endless timelike curves or, separately, past endpoints on pastendless timelike curves - seem both simple and natural. A project to regularize this

1991 Mathematics Subject Classification. 53C50, 83C75.

Key words and phrases. static spacetime, causal boundary, boundary on manifold, Busemann function.

J. L. Flores was supported in part by MCyT-FEDER Grant BFM2001-2871-C04-01, MECyD Grant EX-2002-0612, and MEC Grant RyC-2004-382 and gratefully acknowledges the hospitality of the Department of Mathematics of Saint Louis University. S. G. Harris gratefully acknowledges the hospitality of the Department of Mathematics of the University of Missouri-Columbia. Both authors thank the Isaac Newton Institute for Mathematical Sciences, Cambridge, UK, for support during the Programme on Global Problems in Mathematical Relativity. 
naturality of just the future causal boundary was initiated in [H1]. It was shown there that a complete categorical treatment is possible for the future causal boundary (called there the future chronological boundary): In a category of chronological sets, the addition of the future-chronological (or causal) boundary resulted in a "future-complete" object, and future-completion is both functorial and categorically universal, in the category of chronological sets. In [H2] it was shown how to define a topology on any chronological set, what might be called the future-chronological topology; for a future-completed spacetime, this topology has a number of desirable properties, including giving the right result for the future-completion of $\mathbb{L}^{n}$.

In [H3] it was shown how to construct the future-completion for a standard static spacetime, i.e., a spacetime $V$ conformal to a product spacetime $\mathbb{L}^{1} \times M$, where $M$ is any Riemannian manifold and the conformal factor is independent of $\mathbb{L}^{1}$. The future-completion $V^{+}$of $V$ is most naturally expressed in terms of real-valued functions on $M$, leading to the possibility of imbuing $V^{+}$with a function-space topology. This results in a very simple structure for $V^{+}$as $\mathbb{R}^{1} \times M^{*}$ plus one additional point $\left\{i^{+}\right\}$, where $M^{*}$ is a kind of geometric completion of $M$, adding points at "geometric infinity" (plus the Cauchy completion of $M$, should it not be complete); this geometric completion of $M$ is closely related to the boundary sphere construction for Hadamard manifolds (see [BGS]). The function-space topology on $V^{+}$yields a corresponding topology on $M^{*}$, which may likewise be called the function-space topology there.

However, the function-space topology on $V^{+}=\left(\mathbb{L}^{1} \times M\right)^{+}$is not the futurechronological topology; the latter may have more convergence of sequences than the former. The future-chronological topology on $V^{+}$yields a topology on $M^{*}$; this may be called the chronological topology on $M^{*}$, as the same topology is imparted to $M^{*}$ from using the past-chronological topology on the past-completion $V^{-}$of $V$. Contrary to what was said in [H3], the result of using these topologies may very well be that $V^{+}$does not have the simple product structure over $M^{*}$ that the function-space topology yields; and although $M^{*}$ and $V^{+}$are always Hausdorff in the function-space topology, this may not be true in the future-chronological topology for $V^{+}$and the chronological topology for $M^{*}$. This is a benefit, as the non-Hausdorffness on $V^{+}$is actually better representative of the physical conditions in the spacetime; furthermore, the chronological topology on $M^{*}$ is always compact, which is not so for the function-space topology.

It is the purpose of this paper to examine in detail the circumstances that lead the future-chronological topology to differ from the function-space topology on $V^{+}$. In particular, we show (Theorem 5.15) that if the future-chronological topology on $V^{+}$is not the same as the function-space topology, then the former must be nonHausdorff.

Section 2 provides a recapitulation of the basic framework and definitions, followed by a detailed analysis of the prime example space. This $2+1$ example space is not likely of much physical interest (it could perhaps represent a static spacetime based around an infinite band of dielectric material in an otherwise empty plane), but it presents a sharp focus on the manner in which the geometry of the Riemannian factor can lead to topology in the boundary which may not be naively expected: lack of Hausdorffness and no product structure in the boundary. The paper thereafter follows two nearly independent tracks:

Sections 3 and 4 present a few general results on convergence in the futurechronological topology. Section 3 lays out technical lemmas, and section 4 shows 
how convergence is related to rays (minimizing semi-infinite geodesics).

Sections 5 and 6 present the most important results, many of which are applicable very generally. Notable are Corollary 5.12 (detailing when a sequence of points in any strongly causal spacetime possesses a limit in the future-chronological topology), Theorem 5.14 (showing that adding the boundary to a standard static spacetime compactifies the Riemannian factor in the chronological topology), Theorem 5.15 (showing Hausdorffness is the sole key to the question of whether or not the naive topology - a product topology — is the proper one), Theorem 6.2 (a condition on the Riemannian factor guaranteeing the naive topology, a condition occurring among classical spacetimes), and Corollary 6.6 (an application to such spacetimes as external Schwarzschild, external or internal Reissner-Nordström, or parts of Schwarzschild-de Sitte.).

\section{Preliminaries AND Example}

The following can be found in [GKP], [HE], or [H1]: Let $V$ be a strongly causal, time-oriented spacetime. For any point $p \in V$, the past of $p$ is $I^{-}(p)=\{q \in V \mid q \ll$ $p$; ; for a subset $A \subset V$, the past of $A$ is $I^{-}[A]=\bigcup_{p \in A} I^{-}(p)$. A subset $P \subset V$ is a past set if $I^{-}[P]=P$; it is an indecomposable past set, or IP, if it is not the union of two proper subsets, both being past sets. Any IP can be expressed as $I^{-}[\gamma]$ for $\gamma$ a timelike curve; if $P$ is not the past of any point, then it is the past of a future-endless timelike curve. The future causal boundary of $V$ is $\partial^{+}(V)=\{P \subset$ $V \mid P$ is an IP and is not $I^{-}(p)$ for any $\left.p \in V\right\}$. Let $V^{+}=V \cup \partial^{+}(V)$; then we can define an extension to $V^{+}$of the chronology relation $\ll$ on $V$ as follows: For $p \in V$ and $P, Q \in \partial^{+}(V)$,

$$
\begin{aligned}
& p \ll P \text { iff } p \in P ; \\
& P \ll p \text { iff for some } z \in V \text { with } z \ll p, P \subset I^{-}(z) ; \text { and } \\
& P \ll Q \text { iff for some } z \in V \text { with } z \in Q, P \subset I^{-}(z) .
\end{aligned}
$$

(In a complete treatment of the future causal boundary, additional chronology relations would be defined within $V$ - the process called past determination in [H1] but in the spacetimes of interest for this paper, that is unnecessary, as they are already past-determined. The usage of $V^{+}$here is that of $\widehat{V}$ in [H1]; in [H1] $V^{+}$denotes the further application of past-determination. We will ignore that distinction for this paper, as $\widehat{V}=V^{+}$for the spacetimes considered here.)

As is shown in [H1], this same process applies equally well to any "chronological set": a set $X$, with a transitive and anti-reflexive relation $\ll$, which has a countable subset $D$ such that for any $\alpha \ll \beta$ in $X$, there is some $\delta \in D$ with $\alpha \ll \delta \ll \beta$, and for which no point is unrelated to all other points. The only difference is that "timelike curve" must be replaced by "future chain", which means a sequence of points $\alpha_{1} \ll \cdots \ll \alpha_{n} \ll \alpha_{n+1} \ll \cdots$. (Also: In a spacetime the past of any point is an IP, but there are chronological sets where the past of a point may be decomposable; but no such chronological sets appear in this paper.) A point $\alpha \in X$ is a future limit of the future chain $c=\left\{\alpha_{n}\right\}$ if $I^{-}(\alpha)=I^{-}[c]$; if $X$ is a strongly causal, time-oriented spacetime, this is equivalent to $\alpha$ being the future endpoint of a timelike curve through the points $\left\{\alpha_{n}\right\}$.

The identical process of defining the future causal boundary, $\partial^{+}$, works for a chronological set, and we define $X^{+}=X \cup \partial^{+}(X)$, called the future-completion of $X$, with the same extensions of $\ll$ as above. Call a chronological set futurecomplete if every future chain has a future limit. Then for any chronological set 
$X$,

(1) $X^{+}$is again a chronological set,

(2) $X^{+}$is future-complete,

(3) future-completion is a functor in an appropriate category of chronological sets, and

(4) future-completion is universal, hence, categorically unique for providing a process of future-completing any chronological set (in the language of $[\mathrm{M}]$ : future-completion is left-adjoint to the forgetful functor).

These are the reasons for believing that the future causal boundary is a natural construction.

Although the causality relation is not crucial for study of the effects of the chronological relation, it can add some additional insights to boundary considerations. The causality relation in $V$ can also be extended to $V^{+}$:

$$
\begin{aligned}
& p \prec P \text { iff } I^{-}(p) \subset P ; \\
& P \prec p \text { iff } P \subset I^{-}(p) ; \text { and } \\
& P \prec Q \text { iff } P \subset Q .
\end{aligned}
$$

A static spacetime is one with a timelike Killing field whose perpendicular-space is integrable. A standard static spacetime is a warped product of the form $\mathbb{L}^{1} \times M$ with metric $-\Omega d t^{2}+\bar{h}$, where $\bar{h}$ is a Riemannian metric on $M$ and $\Omega: M \rightarrow \mathbb{R}$ is a positive function (this is always strongly causal and time-orientable). Thus, any standard static spacetime is conformal to a metric product, $\mathbb{L}^{1} \times M$ with metric $-d t^{2}+h$, where $h=(1 / \Omega) \bar{h}$. As the causal boundary construction is conformally invariant (relying solely upon the chronology relation), we will be able to encompass results for all standard static spacetimes even if we restrict our study to metric products. Accordingly, from this point on, the spacetime $V$ will always mean a metric product $\mathbb{L}^{1} \times M$, metric $-d t^{2}+h$.

For any function $f: M \rightarrow \mathbb{R}$, let $\mathrm{P}(f)$ denote the past of the graph of $f$, i.e., $\{(t, x) \in V \mid t<f(x)\}$. It is shown in [H3] that the past sets of $V$ are precisely the set $V$ itself and all subsets of the form $\mathrm{P}(f)$, where $f$ is any Lipschitz-1 function on $M$, i.e., one satisfying $|f(x)-f(y)| \leq d(x, y)$, where $d$ is the distance function on $M$ coming from its Riemannian metric $h$; we can even think of $V$ as $\mathrm{P}(\infty)$. Let $\mathcal{L}_{1}(M)$ denote the set of Lipschitz-1 functions on $M$.

The IPs of $V$ have the form $\mathrm{P}(f)$ for special elements of $\mathcal{L}_{1}(M)$ : For any $t \in \mathbb{R}$ and $x \in M$, let $d_{x}^{t}: M \rightarrow \mathbb{R}$ be the map $d_{x}^{t}(y)=t-d(x, y)$; then for $p=(t, x) \in V$, $I^{-}(p)=\mathrm{P}\left(d_{x}^{t}\right)$. For the elements of $\partial^{+}(V)$ we must look at IPs of the form $I^{-}[\gamma]$ for $\gamma$ a future-endless timelike curve; actually, we can use null curves instead of timelike with no loss of generality. Any null curve $\gamma$ in $V$ can be parametrized so that it is in the form $\gamma(t)=(t, c(t))$ for $c$ a unit-speed curve in $M ; \gamma:[\alpha, \omega) \rightarrow V$ is future-endless precisely if $c$ has no endpoint at $\omega(\omega=\infty$ is allowed). For any endless unit-speed curve $c:[\alpha, \omega) \rightarrow M$, the Busemann function for $c$ is the function $b_{c}: M \rightarrow \mathbb{R}$ given by $b_{c}=\lim _{t \rightarrow \omega} d_{c(t)}^{t}$, i.e.,

$$
b_{c}(x)=\lim _{t \rightarrow \omega} t-d(c(t), x) .
$$

For any $x \in M$, the function $t \mapsto t-d(c(t), x)$ is monotonic increasing, so the limit above always exists, if we allow $\infty$ as a possible limit; but either $b_{c}(x)=\infty$ for all $x$, or $b_{c}(x)$ is finite for all $x$, in which case $b_{c}$ is Lipschitz-1. In either case, $I^{-}[\gamma]=\mathrm{P}\left(b_{c}\right)$. Let $\mathcal{C}_{0}(M)$ be the set of all unit-speed endless curves in $M$ with 
finite Busemann function; then $\partial^{+}(V)$ consists of $\left\{\mathrm{P}\left(b_{c}\right) \mid c \in \mathcal{C}_{0}(M)\right\}$ together with $\mathrm{P}(\infty)$ (i.e., $V$ itself as a single point of the boundary). In parallel with the nomenclature for $\mathbb{L}^{n}$ and other spacetimes, we may call $\mathrm{P}(\infty)$ "timelike infinity" and label it $i^{+}$.

Any ray - a semi-infinite geodesic which is minimizing on all intervals - is always in $\mathcal{C}_{0}(M)$; accordingly, we may call the curves in $\mathcal{C}_{0}(M)$ asymptotically ray-like. If $M$ is a Hadamard manifold - complete, simply connected, and with non-positive curvature - then the rays yield the totality of all finite Busemann functions, and they are used to construct the boundary sphere for $M$; see [BGS]. But positive curvature allows for the existence of asymptotically ray-like curves $c$ such that $b_{c}$ is not the Busemann function of any geodesic (see the example in section 4).

Let $\mathcal{B}(M)$ denote the finite Busemann functions on $M$. Note that for any $c$ : $[\alpha, \omega) \rightarrow M$ in $\mathcal{C}_{0}(M)$, for any $a \in \mathbb{R}$, the curve $c^{a}:[\alpha+a, \omega+a) \rightarrow M$ given by $c^{a}(t)=c(t-a)$ is also in $\mathcal{C}_{0}(M)$ and has $b_{c^{a}}=b_{c}+a$. Thus, there is a natural $\mathbb{R}$-action on $\mathcal{B}(M)$ with $a \cdot b_{c}=b_{c}+a$. Thus we have an $\mathbb{R}$-action on $V^{+}$with $a \cdot(t, x)=(t+a, x), a \cdot \mathrm{P}\left(b_{c}\right)=\mathrm{P}\left(a \cdot b_{c}\right)$, and $a \cdot i^{+}=i^{+}$.

If the metric on $M$ is complete, then all endless curves have infinite length, hence, have $\omega=\infty$. In that case, the chronology relation on $V^{+}$has $p \ll i^{+}$for any $p \in V$ and $p \ll \mathrm{P}\left(b_{c}\right)$ for all $p \in I^{-}\left[\gamma_{c}\right]$ (where $\gamma_{c}(t)=(t, c(t))$ ); there are no other chronology relations involving $\partial^{+}(V)$. (Within $\partial^{+}(V)$ there are causal relations, such as $\mathrm{P}\left(b_{c}\right) \prec i^{+}$and $\mathrm{P}\left(b_{c}\right) \prec a \cdot \mathrm{P}\left(b_{c}\right)$ for $a \geq 0$; there may also be other causal relations.) Simple example: $M=\mathbb{R}^{n}$. For any $x \in \mathbb{R}^{n}$ and any unit vector $u$ in $\mathbb{S}^{n-1}$, we have the ray $c_{u}^{x}(t)=x+t u$; then $b_{c_{u}^{x}}(y)=\langle y-x, u\rangle$, where $\langle-,-\rangle$ denotes the Euclidean inner product; thus, this function is just a shift of $\langle-, u\rangle$. As with any Hadamard manifold, the rays in $\mathbb{R}^{n}$ account for all finite Busemann functions. Thus, aside from the $\mathbb{R}$-action, $\mathcal{B}\left(\mathbb{R}^{n}\right)$ is parametrized by $\mathbb{S}^{n-1}$. This corresponds to the future causal boundary of $\mathbb{L}^{n+1}$ being a null cone on $\mathbb{S}^{n-1}$, as is also seen in the conformal embedding of $\mathbb{L}^{n+1}$ into the Einstein static spacetime $\mathbb{L}^{1} \times \mathbb{S}^{n}$ (see [HE] or [H2]).

If $M$ is incomplete, then there are asymptotically ray-like curves with $\omega$ finite. In that case, besides the same relations that occur when $M$ is complete, for any $c \in \mathcal{C}_{0}(M)$ with $\omega<\infty, \mathrm{P}\left(b_{c}\right) \ll i^{+}$obtains, and some instances of $\mathrm{P}\left(b_{c}\right) \ll p$ for $p \in V$ and of $\mathrm{P}\left(b_{c}\right) \ll \mathrm{P}\left(b_{c^{\prime}}\right)$ will occur. Simple example: With $\bar{M}$ a complete Riemannian manifold and $*$ any point in $\bar{M}$, let $M=\bar{M}-\{*\}$. Let $c:[\alpha, \omega] \rightarrow \bar{M}$ be any finite-length curve with $c(\omega)=*$. Then $c$, restricted to $[\alpha, \omega)$, is in $\mathcal{C}_{0}(M)$ and corresponds to the point $(\omega, *)$ in $\bar{V}=\mathbb{L}^{1} \times \bar{M}$. Thus, the future causal boundary of $V$ is that of $\bar{V}$ together with the timelike line $\mathbb{L}^{1} \times\{*\}$.

What about topology for the boundary? The function space $\mathcal{L}_{1}(M)$ has the compact-open topology; convergence in that topology is the same as pointwise convergence. We can add $\infty$ (as a function) to this topological space by saying that a sequence of functions converges to $\infty$ if and only if the sequence converges pointwise to infinity; this is equivalent to convergence to infinity on any one point. Aside from $i^{+}$, we can identify $\partial^{+}(V)$ with $\mathcal{B}(M)$, a subset of $\mathcal{L}_{1}(M) ; i^{+}$we can identify with $\infty$; and we can identify $V$ with the functions $\mathcal{D}(M)=\left\{d_{x}^{t} \mid t \in \mathbb{R}, x \in M\right\}$, also lying in $\mathcal{L}_{1}(M)$. Then the function-space topology on $V^{+}$is that derived from these identifications, i.e., as $\mathcal{D}(M) \cup \mathcal{B}(M) \cup\left\{i^{+}\right\}$; the induced topology on $V$ is just the manifold topology. In this topology, the $\mathbb{R}$-action on $V^{+}$is continuous; this action is degenerate on $i^{+}$, but the action is free on $V_{0}^{+}=V^{+}-\left\{i^{+}\right\}$(let $\partial_{0}^{+}(V)=\partial^{+}(V)-\left\{i^{+}\right\}$, so $V_{0}^{+}=V \cup \partial_{0}^{+}(V)$, corresponding to $\left.\mathcal{D}(M) \cup \mathcal{B}(M)\right)$. 
Let $M^{*}=V_{0}^{+} / \mathbb{R}$, with the quotient topology (function-space topology on $V_{0}^{+}$); then $\pi: V_{0}^{+} \rightarrow M^{*}$ is a line bundle. Indeed, it is a trivial line bundle: If we choose any point $x \in M$ and define the map $e: \mathcal{L}_{1}(M) \rightarrow \mathbb{R}$ as evaluation at $x$, $e(f)=f(x)$, then $e$ is continuous in the function-space topology. Thus, thinking of $V_{0}^{+}$in its guise as a subset of $\mathcal{L}_{1}(M)$, we get a continuous cross-section $\zeta$ : $M^{*} \rightarrow V_{0}^{+}$of $\pi$ via $\zeta([f])=f-e(f)$ (where $[f]$ denotes the equivalance class of $f$ under the real action) and a continuous bijection $\phi: \mathbb{R} \times M^{*} \rightarrow V_{0}^{+}$via $\phi(a,[f])=a \cdot \zeta([f]) ;$ as $\phi^{-1}(f)=(e(f),[f])$, we see $\phi^{-1}$ is evidently continuous, so this is a homeomorphism. If we restrict $\pi$ to $V$, we just have the obvious projection $\pi: \mathbb{L}^{1} \times M \rightarrow M$. The upshot is that $M^{*}$ is $M$ with a sort of boundary attached, $\partial_{0}^{+}(V) / \mathbb{R}$, i.e., $\mathcal{B}(M) / \mathbb{R}$; we can call this the Busemann boundary of $M$, denoted $\partial_{B}(M)$. (If $M$ is a Hadamard manifold, then this is precisely the boundary sphere of $M$, as explicated, for instance, in [BGS].) We thus have that, in the functionspace topology, $\partial_{0}^{+}(V)=\mathbb{R} \times \partial_{B}(M)$; if $M$ is complete then each line $\mathbb{R} \times\left\{\left[b_{c}\right]\right\}$ is a null line, and adding in $\left\{i^{+}\right\}$, we have that $\partial^{+}(V)$ is a null cone on $\partial_{B}(M)$.

But the function-space topology on $V^{+}$is not the one that is important for understanding the chronological structure of $V$ in the future; and for all that the function-space topology leads to a simple product-structure for $\partial_{0}^{+}(V)$, it may not be entirely reflective of the physics of the spacetime. In [H2] it was shown how to formulate a topology — what we might call the future-chronological topology - on any chronological set $X$. It has a number of desirable properties: If $X$ is a strongly causal spacetime, this results in the manifold topology; using this topology for the future-completion $X^{+}$of the spacetime, $\partial^{+}(X)$ is closed in $X^{+}, X$ is dense in $X^{+}$, and the subspace topology on $X$ induced from that on $X^{+}$is, again, the manifold topology. Future limits of future chains are topological limits in this topology. If $X=\left(\mathbb{L}^{n}\right)^{+}$, this topology is the same as that coming from the conformal embedding of $\mathbb{L}^{n}$ into $\mathbb{L}^{1} \times \mathbb{S}^{n-1}$. In the topological category of chronological sets with spacelike future boundaries, using the future-chronological topology, future-completion retains the functorial, natural, and universal qualities it has in the category of chronological sets. These results are contained in [H2], section 2 (section 5.1 for $\left.\mathbb{L}^{n}\right)$.

The future-chronological topology is defined by means of a limit-operator on sequences: For $X$ a chronological set, for any sequence $\sigma$ of points $\left\{\alpha_{n}\right\}$ in $X, \hat{L}(\sigma)$ is to be thought of as first-order limits of $\sigma$. More concretely, define a set $A$ to be closed if $\hat{L}(\sigma) \subset A$ for every sequence $\sigma$ contained in $A$. So long as a limitoperator $L$ obeys the property that for any subsequence $\tau \subset \sigma, L(\tau) \supset L(\sigma)$, this method defines a topology in which $L(\sigma)$ consists of limits of $\sigma$ (technically, these are the first-order limits). Let $X$ be a chronological set in which the past of every point is indecomposable (this includes both spacetimes and future-completions of spacetimes); then the future-chronological limit-operator in $X$ is defined thus: $\alpha \in$ $\hat{L}\left(\left\{\alpha_{n}\right\}\right)$ if and only if

(1) for all $\beta \in I^{-}(\alpha), \beta \ll \alpha_{n}$ for $n$ sufficiently large, and

(2) for any IP $P \supsetneq I^{-}(\alpha), P$ contains an element $\beta$ such that $\beta \nless \alpha_{n}$ for $n$ sufficiently large.

(If $X$ contains some $\alpha$ with $I^{-}(\alpha)$ decomposable, then a more complicated definition is needed, but the idea is largely the same.)

So long as $X$ is past-distinguishing (i.e., $x \neq y$ implies $\mathrm{P}(x) \neq \mathrm{P}(y)$; this includes any strongly causal spacetime or its future-completion), points are closed in $X$; but 
$X$ need not be Hausdorff. Indeed, it is possible that $\hat{L}(\sigma)$ may contain more than one point - and that represents an important piece of physical information in $X$. Conceivably, $\hat{L}(\sigma)$ might contain an entire sequence $\tau$ of points, in which case $\hat{L}(\tau)$ would be second-order limits of $\sigma$; but no examples of such a chronological set have come to light, and that possibility plays little role in things (it just means that a very few of the proofs of how $\hat{L}$ works must invoke transfinite induction).

In this paper we are concerned only with the application of the future-chronological topology to $V^{+}$for $V=\mathbb{L}^{1} \times M$. As above, let us identify $V_{0}^{+}$with the appropriate subset of $\mathcal{L}_{1}(M)$, i.e, $\mathcal{D}(M) \cup \mathcal{B}(M)$. We can also include $i^{+}$in the discussion by treating that as the function $\infty$; let us call this class of functions $\mathcal{I P}(M)$ (as they represent the IPs of $\mathbb{L}^{1} \times M$ ), with $\mathcal{I P}_{0}(M)=\mathcal{D}(M) \cup \mathcal{B}(M)$ denoting the finite-valued ones. Then we have the following characterization of the future-chronological topology in $V^{+}$from [H3] (a derivation of this is indicated in section 5):

Future-Chronological Topology in $\left(\mathbb{L}^{1} \times M\right)^{+}$. Let $\sigma=\left\{f_{n}\right\}$ be a sequence of functions in $\mathcal{I P}(M)$. Then $\hat{L}(\sigma)$ consists of all $f \in \mathcal{I P}(M)$ satisfying

(1) $f \leq \liminf _{n \rightarrow \infty} f_{n}$ and

(2) for all $g \in \mathcal{I} \mathcal{P}(M)$ with $f \leq g \leq \limsup _{n \rightarrow \infty} f_{n}, g=f$.

With this topology, we again have that the $\mathbb{R}$-action on $V^{+}$is continuous, so we can still consider the projection $\pi: V_{0}^{+} \rightarrow M^{*}$; only now $M^{*}=V_{0}^{+} / \mathbb{R}$ must be imbued with the quotient topology from the future-chronological topology on $V_{0}^{+}$. Let us call this the chronological topology on $M^{*}$. (We could just as easily have time-reversed everything, applying the past-chronological topology-with an appropriate $\check{L}$ operator - to $V^{-}$, the past-completion of $V$. The Busemann functions obtained thereby are just the negatives of the ones for $V^{+}$, and essentially the same structure is obtained for the quotient $M^{*}$.)

The future-chronological topology on $V^{+}$differs from the function-space topology in that more sequences may be convergent: If $f \in \mathcal{I P}(M)$ is the pointwise limit of a sequence $\left\{f_{n}\right\}$ in $\mathcal{I P}(M)$, then $f \in \hat{L}\left(\left\{f_{n}\right\}\right)$; but the converse does not necessarily hold. The function-space topology is always Hausdorff, but the future-chronological topology may not be; and this difference passes through to the quotient $M^{*}$ as well. When there is some $f \in \hat{L}\left(\left\{f_{n}\right\}\right)$ which is not the pointwise limit of the sequence, there must be some $x \in M$ with $f(x) \neq \lim f_{n}(x)$, which means that the $x$-based evaluation map $e: V_{0}^{+} \rightarrow \mathbb{R}$ is not continuous; thus, $V_{0}^{+}$, in the future-chronological topology, may not be a product over $M^{*}$ in the chronological topology (contrary to what was said in [H3]).

These important features of the chronological topology will be illustrated in our prime example space (Example 4 in [H3]):

\section{The Unwrapped Grapefruit on a Stick.}

Let $G$ be a grapefruit on a stick: a sphere transfixed symmetrically by an infinite cylinder of smaller radius. For definiteness, take the sphere to be the unit sphere at the origin of $\mathbb{R}^{3}$ and the cylinder to be $\left\{x^{2}+y^{2}=r^{2}\right\}$ for some fixed positive $r<1$, best imagined as small; the surface $G$ is that portion of the sphere outside the cylinder combined with that portion of the cylinder outside the sphere. This is not differentiable where the cylinder meets the sphere, at $z= \pm r^{\prime}\left(\right.$ where $r^{\prime}=\sqrt{1-r^{2}}$ ), 
so we must introduce a smoothing, say between $z=r^{\prime}$ and $z=r^{\prime}-\delta$ for some positive $\delta$ much smaller than $r^{\prime}$, and also between $z=-r^{\prime}$ and $z=-\left(r^{\prime}-\delta\right)$.

Let $M=\tilde{G}$, the universal cover of $G$. We can think of $M$ as parametrized by rectangular coordinate $z$ and polar-angle coordinate $\theta$; it is a plane which is flat for $|z|>r^{\prime}$ but with uniform positive curvature in the region $|z|<r^{\prime}$ (actually, the uniform positive curvature is in $|z|<r^{\prime}-\delta$, with some negative curvature appearing where $r^{\prime}-\delta<|z|<r^{\prime}$; but this has essentially no practical effect on the asymptotic geometry of the space). Specifically, the metric in the flat part is $r^{2} d \theta^{2}+d z^{2}$, while in the round part it is $\left(1-z^{2}\right) d \theta^{2}+\left(1 /\left(1-z^{2}\right)\right) d z^{2}$ (with smoothing between those two in the negative-curvature part).

Geodesics in $M$ are most easily seen by looking in $G$. A typical geodesic in the cylinder is, of course, a helix; where it encounters the sphere, it turns into a great circle and thus travels from one intersection of sphere with cylinder to the other, upon which encounter it again becomes a helix. In $M$, this is a straight line in the flat part, turning into a curve through the round part that traverses no more than $\pi$ in the $\theta$ coordinate before it again becomes a line in the other flat part. Clearly, such a geodesic will be minimizing. Other geodesics will not be minimizing, save for short arcs: geodesics which are endlessly repeated great circles in the sphere of $G$, corresponding to periodically oscillating curves in the round part of $M$. So what is a minimizing geodesic between two points in the round part of $M$, separated by a large amount of $\theta$ (say, much greater than $\pi$ )? Ask the question in $G$ : How may one travel efficiently between two points on the grapefruit, while requiring that one make several revolutions before stopping? The clear answer is to travel quickly off the grapefruit and wrap around the stick the requisite number of times (thus accomplishing the necessary increase in $\theta$ ) before returning to the grapefruit to reach the endpoint. Translated to $M$, this is (approximately) a curve which goes from the round part to the flat part, then holds at $z= \pm r^{\prime}$ for some distance, then goes back into the round part. In actuality, the curve never reaches the flat part, but stays for a long distance in the negative-curvature part; but the flat and negative-curvature metrics hardly differ at all in measuring the length of such a curve. (There are also geodesics which are the limits of those minimizers between distant points in the round part, as one endpoint is fixed and the other is pushed out to infinity: They start in the round part, enter the negative-curvature region, and remain there, asymptotic to the flat part.) Consideration of these minimizing geodesics allows calculation (at least in asymptotic form, for large differences in $\theta$ ) of the distance function in $M$.

It will be useful to examine the functions $d_{x}^{t}$ for $x$ sitting in the round part; in particular, we will want to consider the sequence $\sigma$ in $V$ consisting of $\sigma(n)=\left(t_{n}, x_{n}\right)$ for $t_{n}=r n+(1-r) \pi / 2$ and $x_{n}=\left(n, z_{0}\right)$ in $\theta-z$ coordinates for fixed $z_{0}$ with $\left|z_{0}\right|<r^{\prime}$ (actually, $\left|z_{0}\right|<r^{\prime}-\delta$, but we will henceforth ignore that minor correction). It will be shown that $\sigma$ converges to two points in $\partial_{0}^{+}(V)$, using the future-chronological topology, but has no limit in $V^{+}$using the function-space topology. This corresponds to the sequence $\left\{x_{n}\right\}$ in $M$ having two limits in $\partial_{B}(M)$ using the chronological topology but having no limit in $M^{*}$ using the function-space topology. The manner of convergence will show that in the future-chronological topology, $V_{0}^{+}$does not have a simple product structure over $M^{*}$.

Rather than give the minutiae of the asymptotic formulas for $d_{x_{n}}^{t_{n}}$, we will just display the limit for $n$ going to infinity, which is all that we are are interested in. Define the function $f(z)=\sin ^{-1}\left(z / r^{\prime}\right)-r \sin ^{-1}\left(r z /\left(r^{\prime} \sqrt{1-z^{2}}\right)\right)$; this is a strictly 
increasing function taking the interval $\left[-r^{\prime}, r^{\prime}\right]$ onto $[-(1-r) \pi / 2,(1-r) \pi / 2]$. Let $\bar{f}$ be the extension of $f$ to all of $\mathbb{R}$ defined by $\bar{f}(z)=(1-r) \pi / 2$ for $z>r^{\prime}$ and $\bar{f}(z)=-(1-r) \pi / 2$ for $z<-r^{\prime}$. Then $\lim d_{x_{n}}^{t_{n}}=\delta_{z_{0}}$ is given by

$$
\delta_{z_{0}}(\theta, z)=r \theta+\left|\bar{f}(z)+f\left(z_{0}\right)\right|-(1-r) \pi / 2 .
$$

Our goal is to see how this compares with elements of $\partial^{+}(V)$.

The geodesics in $M$ come mostly in three classes: those eventually in $z>r^{\prime}$, those eventually in $z<-r^{\prime}$, and the oscillating ones remaining within $|z|<r^{\prime}$. (There are also those geodesics asymptotic to the first two classes, remaining eventually within the negative-curvature region.) The first two classes are all in $\mathcal{C}_{0}(M)$ and are essentially straight lines in the flat portions of $M$. But those in the third class, being non-minimizing over any interval longer than $\pi$, do not go efficiently to infinity, and they all have infinite Busemann functions; in other words, the null geodesics in $V$ sitting over these oscillatory geodesics in $M$ each have the entire spacetime $V$ for their pasts, as they represent travellers who, while going out to infinity, do so in a dilatory manner, so that they eventually see all events. (The geodesics asymptotic to the first two classes have Busemann functions the same as $z=$ constant geodesics in the respective classes.)

The straight-line geodesics in $M$ have basically the same Busemann functions as lines in the Euclidean plane; the only difference for, say, the line $c_{k}$ of slope $k$ given by $z=k \theta$ (or anything parallel to that) with $k$ positive and defined for $\theta>0$, is that $b_{c_{k}}(\theta, z)$ has an additional constant added to it (in comparison with the Busemann function for that line in the flat plane) for those $z<-r^{\prime}$-representing the additional distance it takes to get over the hump of the grapefruit, compared to the flat plane - and is rather complicated for those $z$ with $|z|<r^{\prime}$. What is truly interesting is what happens for a line of zero slope: This includes geodesics in both of the two $\mathcal{C}_{0}(M)$ classes, yielding similar but subtly different Busemann functions. Let $c^{+}$be the geodesic $c^{+}(t)=((1 / r) t, 1)$ and $c^{-}$its twin in the other class, $c^{-}(t)=((1 / r) t,-1)$. Then we have

$$
\begin{aligned}
& b_{c^{+}}(\theta, z)=r \theta+\bar{f}(z)-(1-r) \pi / 2 \\
& b_{c^{-}}(\theta, z)=r \theta-\bar{f}(z)-(1-r) \pi / 2 .
\end{aligned}
$$

The curious aspect of these Busemann functions is that although they are not $\mathbb{R}$ related to one another, i.e, $\left[b_{c^{+}}\right] \neq\left[b_{c^{-}}\right]$, they remain a bounded distance apart (as $\bar{f}$ is bounded); this is not so for any of the other Busemann functions in the space, nor for those in Euclidean space, nor, it appears, for most geodesics in most spaces. One implication of this is that the null lines generated by $b_{c+}$ and $b_{c^{-}}$in $\partial^{+}(V)$ have a most unusual leap-frog kind of relationship: If $\alpha$ is any element of the first line and $\beta$ any element of the second, then there is some $s_{0} \in \mathbb{R}$ such that $\alpha \prec s \cdot \beta$ for all $s \geq s_{0}$ and $\alpha \succ s \cdot \beta$ for all $s \leq s_{0}-(1-r) \pi$.

Now compare with $\delta_{z_{0}}$ : Note first that $\delta_{z_{0}}=\max \left\{b_{c^{+}}+f\left(z_{0}\right), b_{c^{-}}-f\left(z_{0}\right)\right\}$. The import of this is that $\mathrm{P}\left(\delta_{z_{0}}\right)$ is not an IP: It is decomposable as $\mathrm{P}\left(f\left(z_{0}\right) \cdot b_{c^{+}}\right) \cup$ $\mathrm{P}\left(-f\left(z_{0}\right) \cdot b_{c^{-}}\right)$. This means that $\delta_{z_{0}}$ is not in $\mathcal{B}(M)$; thus, the sequence $\sigma$ has no limit in $V^{+}$with the function-space topology. In similar vein, the sequence of points $\left\{x_{n}\right\}=\left\{\left(n, z_{0}\right)\right\}$ in $M$ has no limit in $M^{*}$ with the function-space topology; indeed, no subsequence has a limit, either, so $M^{*}$ is not compact in this topology.

On the other hand, from $\lim _{n \rightarrow \infty} d_{x_{n}}^{t_{n}}=\max \left\{b_{c^{+}}+f\left(z_{0}\right), b_{c^{-}}-f\left(z_{0}\right)\right\}$ and the fact that no other element of $\partial^{+}(V)$ comes in between $\delta_{z_{0}}$ and, on the one 
hand, $b_{c^{+}}+f\left(z_{0}\right)$, or, on the other hand, $b_{c^{-}}-f\left(z_{0}\right)$, it follows that both of these functions are future-chronological limits of the sequence $\left\{d_{x_{n}}^{t_{n}}\right\}$, i.e., $\hat{L}(\sigma)=$ $\left\{b_{c^{+}}+f\left(z_{0}\right), b_{c^{-}}-f\left(z_{0}\right)\right\}$. Thus, we have additional convergence in $V^{+}$in the future-chronological topology, but at a price: It is not Hausdorff. (This price must always be paid, as will be shown in section 5.) We also have convergence in $M^{*}$ with the chronological topology: $\left\{\left(n, z_{0}\right)\right\}$ converges to both $\left[b_{c^{+}}\right]$and $\left[b_{c^{-}}\right]$.

The lack of Hausdorffness in $V^{+}$using the future-chronological topology should not be seen as a defect: It accurately reflects the physics of the universe being modeled, in that there are observers with pasts that are not identical and with neither containing the other, but are none the less closely related. In $M^{*}$, the lack of Hausdorffness indicates that there are pairs of asymptotically ray-like curves which go out to infinity in nearly the same direction, but are subtly different in the asymptotics of their respective distance functions.

A final issue: Can $\pi: V_{0}^{+} \rightarrow M^{*}$ be a trivial bundle, i.e., can $V_{0}^{+}$be realized as $\mathbb{R} \times M^{*}$ in an $\mathbb{R}$-covariant manner? If this were possible, then there would be an $\mathbb{R}$-covariant map $\tau: V_{0}^{+} \rightarrow \mathbb{R}$, i.e., one such that $\tau(a \cdot \alpha)=\tau(\alpha)+a$, continuous with the future-chronological topology on $V_{0}^{+}$. But if such a map existed, then we would have

$$
\lim _{n \rightarrow \infty} \tau(\sigma(n))=\tau\left(b_{c^{+}}+f\left(z_{0}\right)\right)=\tau\left(b_{c^{+}}\right)+f\left(z_{0}\right)
$$

and also

$$
\lim _{n \rightarrow \infty} \tau(\sigma(n))=\tau\left(b_{c^{-}}-f\left(z_{0}\right)\right)=\tau\left(b_{c^{-}}\right)-f\left(z_{0}\right)
$$

from which we derive $\tau\left(b_{c^{-}}\right)-\tau\left(b_{c^{+}}\right)=2 f\left(z_{0}\right)$. But the left side is independent of $z_{0}$, while the right side is not; thus, no such $\tau$ can exist. Thus, $V_{0}^{+}$is not a simple product over $M^{*}$. (This appears generally to be the case when the two topologies differ, though details are somewhat murky.)

But it is worth noting that $V_{0}^{+}$, in the future-chronological topology is, none the less, a line bundle over $M^{*}$ in the chronological topology, though it is not a trivial line bundle. (It is unclear to what extent this generalizes to other standard static spacetimes.) Showing the details of this will help illuminate the nature of the future-chronological topology.

First some general points. For local trivialization, we need open sets in $M^{*}$. In the quotient topology, an open set in $M^{*}$ is the image under $\pi$ of an $\mathbb{R}$-invariant open set in $V_{0}^{+}$, and likewise for a closed set. Note that for any point $f \in V_{0}^{+}$ (thought of as $\mathcal{I P}_{0}(M)$ ), the $\mathbb{R}$-orbit $\mathbb{R} \cdot f$ is a closed set: For any sequence of reals $\left\{a_{n}\right\}$, if $g \in \mathcal{I P}_{0}(M)$ satisfies $g \leq \liminf \left(f+a_{n}\right)$ and if there is no other $g^{\prime} \in \mathcal{I P}_{0}(M)$ with $g \leq g^{\prime} \leq \lim \sup \left(f+a_{n}\right)$, then it must be that $\left\{a_{n}\right\}$ has a limit $a$ and $g=f+a$. For let $a^{-}=\liminf a_{n}$ and $a^{+}=\lim \sup a_{n}$. We have $g \leq f+a^{-}$ (so we know $a^{-} \neq-\infty$, if we are assuming $\hat{L}\left(\left\{f+a_{n}\right\}\right) \neq \emptyset$ ). If $a^{+}>a^{-}$, then $g^{\prime}=f+b$ violates the property of $g$ for any $b$ with $a^{-}<b \leq a^{+}$; thus, we know that $a^{+}=a^{-}$, so there is a limit $a$. And $a$ must be finite, since if $a=\infty$, then $g^{\prime}=g+1$ violates the property for $g$. We have $g \leq f+a$; if $g \neq f+a$, then $f+a$ violates the property of $g\left(g \leq f+a=\lim \sup \left(f+a_{n}\right)\right)$. Therefore, $g=f+a$. It follows that for any sequence $\sigma \subset \mathbb{R} \cdot f, \hat{L}(\sigma)$ is either empty (the sequence heads to $-\infty)$ or consists of a single element of the same $\mathbb{R}$-orbit: $\mathbb{R} \cdot f$ is closed in $V_{0}^{+}$with the future-chronological topology. (In $V^{+}$, the closure of $\mathbb{R} \cdot f$ is $(\mathbb{R} \cdot f) \cup\left\{i^{+}\right\}$.) It follows that $\{[f]\}$ is closed in $M^{*}$ with the chronological topology, i.e., points are closed in this topology. 
Back to the unwrapped grapefruit on a stick, $M=\tilde{G}$ : There are two pairs of troublesome non-Hausdorff-related points, $\left\{\left[b_{c^{+}}\right],\left[b_{c^{-}}\right]\right\}$and the analogous points for going in the opposite direction, i.e., generated by the curves $-c^{+}(t)=(-(1 / r) t, 1)$ and $-c^{-}(t)=(-(1 / r) t,-1)$. Remove one from each pair to form the trivializing open sets: Let $U^{-}=M^{*}-\left\{\left[b_{c^{+}}\right],\left[b_{-c^{+}}\right]\right\}$and let $U^{+}=M^{*}-\left\{\left[b_{c^{-}}\right],\left[b_{-c^{-}}\right]\right\}$; as points are closed, these are open sets, and they clearly cover $M^{*}$. Let $W^{-}=\pi^{-1}\left[U^{-}\right]$and $W^{+}=\pi^{-1}\left[U^{+}\right]$. Pick a point $x=(\theta, z) \in M$ and let $e_{x}: V_{0}^{+}=\mathcal{I P}_{0}(M) \rightarrow \mathbb{R}$ be evaluation at $x$; is $e_{x}$ continuous on either $W^{-}$or $W^{+}$? It depends on the $z$ chosen for $x$. For the sequence $\left\{d_{x_{n}}^{t_{n}}\right\}$ above (with $\left.x_{n}=\left(n, z_{0}\right)\right),\left\{e_{x}\left(d_{x_{n}}^{t_{n}}\right)\right\}$ converges to $e_{x}\left(b_{c^{+}}+f\left(z_{0}\right)\right)$ precisely when $z \geq-z_{0}$, and it converges to $e_{x}\left(b_{c^{-}}-f\left(z_{0}\right)\right)$ when $z \leq-z_{0}$. The problem for continuity is that $\left\{d_{x_{n}}^{t_{n}}\right\}$ has two limits in $V_{0}^{+}, b_{c^{+}}+f\left(z_{0}\right)$ and $b_{c^{-}}-f\left(z_{0}\right)$. In $W^{-}$, we don't have to worry about convergence to $b_{c^{+}}+f\left(z_{0}\right)$, as that is missing in $W^{-}$, so we are at liberty to choose, say, $z=-1$; then $z<-z_{0}$ for all possible choices of troublesome sequences $\left\{d_{x_{n}}^{t_{n}}\right\}$, so that $e_{x}: W^{-} \rightarrow \mathbb{R}$ will be continuous. This provides a cross-section $\zeta^{-}: U^{-} \rightarrow W^{-}$of $\pi$ defined by $\zeta^{-}([f])=f-e_{x}(f)$. Similarly, a choice of $x$ with $z=1$ yields a cross-section $\zeta^{+}: U^{+} \rightarrow W^{+}$.

In short: The base space in the line bundle $\pi: V_{0}^{+} \rightarrow M^{*}$ is non-Hausdorff, but the bundle is non-trivial because the non-Hausdorffness is not reflected in the total space in an $\mathbb{R}$-invariant manner. Rather, for pairs $\left\{x^{+}, x^{-}\right\}$in the base space which are non-Hausdorff-related, for any element $f^{+}$in the fibre over $x^{+}$, only some of the elements in the fibre over $x^{-}$are non-Hausdorff-related to $f^{+}$. But away from non-Hausdorff-related elements, the bundle is trivial.

\section{Technical Results on Convergence}

This section presents some preliminary work of a technical nature, needed for the more interesting results on convergence later in the paper. As throughout the remainder of this paper, $M$ will be an arbitrary Riemannian manifold, $V=\mathbb{L}^{1} \times M$, and $\omega$ will commonly be used for the limiting argument of an endless unit-speed curve (necessarily $\omega=\infty$ if $M$ is complete).

Suppose that $c$ is a ray in $M$ (unit-speed, as all curves considered here will be), i.e., for all $t \geq s \geq 0, d(c(s), c(t))=t-s$. It then eaisly follows that for all $s \geq 0$, $b_{c}((c(s)))=s$. If $c$ is asymptotically ray-like (i.e., lies in $\mathcal{C}_{0}(M)$, i.e., has a finite Busemann function), then the same thing is asymptotically true:

Lemma 3.1. For any $c \in \mathcal{C}_{0}(M), \lim _{s \rightarrow \omega}\left(b_{c}(c(s))-s\right)=0$.

Proof. It suffices if we show that $d(c(s), c(t))$ is arbitrarily close to $t-s$ for $t \geq s$ and $s$ sufficiently large. If this is not so, then for some $\epsilon>0$, there is a sequence $\left\{s_{n}\right\}$ increasing to $\omega$ with the propety that for all $n$,

$$
d\left(c\left(s_{2 n-1}\right), c\left(s_{2 n}\right)\right) \leq s_{2 n}-s_{2 n-1}-\epsilon .
$$

It follows that

$$
d\left(c\left(s_{0}\right), c\left(s_{2 n}\right)\right) \leq s_{2 n}-s_{0}-n \epsilon .
$$

But then $b_{c}\left(c\left(s_{0}\right)\right)=\lim \left(s_{2 n}-d\left(c\left(s_{0}\right), c\left(s_{2 n}\right)\right)\right) \geq \lim \left(s_{0}+n \epsilon\right)=\infty$, violating the asymptotic ray-like nature of $c$.

We will want to compare the asymptotic behavior of the Busemann function of a curve $c$, possibly in other directions, such as another curve $c^{\prime} \in \mathcal{C}_{0}(M)$, to other 
relevant functions: the pointwise limits of functions in $\mathcal{D}(M)$. Let $\overline{\mathcal{D}}(M)$ denote the closure of $\mathcal{D}(M)$ in $\mathcal{L}_{1}(M)$ with the function-space topology; this includes $\mathcal{B}(M)$, but may (depending on $M$ ) also include functions representing past sets that are not IPs but are closely related to IPs (such as the functions $\delta_{z_{0}}$ for $M=\tilde{G}$, the example in section 2).

Definition. For any $c$ and $c^{\prime}$ in $\mathcal{C}_{0}(M)$ and any continuous $f: M \rightarrow \mathbb{R}, c$ is $c^{\prime}$ asymptotically like $f$ if $\lim _{s \rightarrow \omega^{\prime}}\left(b_{c}\left(c^{\prime}(s)\right)-f\left(c^{\prime}(s)\right)\right)=0\left(\omega^{\prime}\right.$ the limiting argument for $\left.c^{\prime}\right)$, i.e., $b_{c}-f$ goes to 0 along $c^{\prime}$.

Generally, this will be used only for functions $f \in \overline{\mathcal{D}}(M)$, though the definition can apply more broadly.

Comparing $b_{c}$ with such a function $f$ is particularly simple along the direction $c$ itself, as we just need to check that $f$ fulfills the role of $b_{c}$ in Lemma 3.1 ; and when this asymptotic relationship obtains, $b_{c}$ is actually bounded by $f$ :

Lemma 3.2. For any $c \in \mathcal{C}_{0}(M)$ and $f \in \overline{\mathcal{D}}(M)$, the following are equivalent:

(1) c is c-asymptotically like $f$;

(2) $\lim _{s \rightarrow \omega}(f(c(s))-s)=0$;

(3) for some sequence $\left\{s_{k}\right\}$ increasing to $\omega, \lim _{k \rightarrow \infty}\left(f\left(c\left(s_{k}\right)\right)-s_{k}\right)=0$.

Furthermore, when any of these obtain, $b_{c} \leq f$ on $M$.

Proof. In light of Lemma 3.1, all we need to show for the first part is that (3) implies (2). Let $f_{c}=f \circ c$, a real-valued function defined on $[\alpha, \omega)$, the domain of $c$. Note that since $f \in \mathcal{L}_{1}(M)$ and $c$ is unit-speed, $f_{c}$ is Lipschitz-1 also. Let $\delta:[\alpha, \omega) \rightarrow \mathbb{R}$ be defined by $\delta(s)=f_{c}(s)-s$. We know $\delta$ goes to 0 on the sequence $\left\{s_{k}\right\}$, and we wish to show that $\delta$ goes to 0 generally. Because $\left\{s_{k}\right\}$ goes out to $\omega$, this follows directly from showing that $\delta$ is monotonic decreasing:

Suppose $t \geq s$. Then $f_{c}(t)-f_{c}(s) \leq t-s$ (since $f_{c}$ is Lipschitz-1), so $f_{c}(t) \leq$ $f_{c}(s)+t-s=\delta(s)+t$. Therefore, $f_{c}(t)-t \leq \delta(s)$, or $\delta(t) \leq \delta(s)$. This finishes the first part.

For the second part, suppose that (2) holds and that $f$ is the pointwise limit of a sequence $\left\{d_{x_{n}}^{t_{n}}\right\}$ in $\mathcal{D}(M)$. Let $x$ be an arbitrary point in $M$. We will show that for any $\epsilon>0$, for $s$ sufficiently large, $f(x) \geq s-d(c(s), x)-\epsilon$; that will establish that $f(x) \geq b_{c}(x)$.

For $n$ sufficiently large (depending on $x$ and $\epsilon$ ), $\left|f(x)-d_{x_{n}}^{t_{n}}(x)\right|<\frac{\epsilon}{3}$. Therefore, we have, for any $s<\omega$,

$$
\begin{aligned}
f(x) & \geq d_{x_{n}}^{t_{n}}(x)-\frac{\epsilon}{3} \\
& =t_{n}-d\left(x_{n}, x\right)-\frac{\epsilon}{3} \\
& \geq t_{n}-d\left(x_{n}, c(s)\right)-d(c(s), x)-\frac{\epsilon}{3} \\
& =d_{x_{n}}^{t_{n}}(c(s))-d(c(s), x)-\frac{\epsilon}{3} \\
& =d_{x_{n}}^{t_{n}}(c(s))-f(c(s))+f(c(s))-s+s-d(c(s), x)-\frac{\epsilon}{3} .
\end{aligned}
$$

From our hypothesis of (2), if we choose $s$ sufficiently large, then $|f(c(s))-s|<\frac{\epsilon}{3}$. For any fixed such $s$, we can require that $d_{x_{n}}^{t_{n}}$ be close to $f$ at both $x$ and $c(s)$, i.e., 
that for $n$ sufficiently large, we also have, $\left|d_{x_{n}}^{t_{n}}(c(s))-f(c(s))\right|<\frac{\epsilon}{3}$. Putting these inequalities into (3.1) results in

$$
f(x) \geq s-d(c(s), x)-\epsilon
$$

as desired.

In Lemma 3.2, the functions $f$ we considered for comparison with Busemann functions were specified as those in the $\mathcal{L}_{1}(M)$-closure of $\mathcal{D}(M)$, i.e., the functionspace closure of $V$. Of course, that also includes anything in the closure of $V_{0}^{+}$-i.e., $\mathcal{D}(M) \cup \mathcal{B}(M)$ - as the elements of $\mathcal{B}(M)$ are part of the closure of $\mathcal{D}(M)$ in $\mathcal{L}_{1}(M)$; that is to say, any function expressible as a limit of elements of $\mathcal{D}(M) \cup \mathcal{B}(M)$ is also expressible as a limit of elements purely of $\mathcal{D}(M)$. As a matter of practicality, it is worth noting just how this can be implemented; this is the content of the next lemma.

Standard techniques, such as covering a compact set with open balls, easily show that among Lipschitz-1 functions, uniform convergence on compact subsets is equivalent to pointwise convergence (and uniform convergence on compact subsets is equivalent to convergence in the compact-open topology, even for merely continuous functions). This is useful for showing the following.

Lemma 3.3. Let $\left\{c_{n}\right\}$ be a sequence of curves in $\mathcal{C}_{0}(M)$. Then there is a sequence of numbers $\left\{s_{n}\right\}$ such that $\left\{b_{c_{n}}\right\}$ converges pointwise to some function $f$ if and only if $\left\{d_{c_{n}\left(s_{n}\right)}^{s_{n}}\right\}$ converges pointwise to $f$. Furthermore, the same is true with respect to the future-chronological topology, i.e., $\hat{L}\left(\left\{b_{c_{n}}\right\}\right)=\hat{L}\left(\left\{d_{c_{n}\left(s_{n}\right)}^{s_{n}}\right\}\right)$.

Proof. Let $\left\{K_{n}\right\}$ be an exhaustion of $M$ by compact subsets, $K_{n} \subset \operatorname{interior}\left(K_{n+1}\right)$. Since each $b_{c_{n}}$ is the pointwise limit of $\left\{d_{c_{n}(s)}^{s}\right\}$ as $s$ goes to $\omega_{n}$ (the limiting argument for $c_{n}$ ), we also have uniform convergence on $K_{n}$ : For each $n$, there is some $s_{n}<\omega_{n}$ such that $\left|b_{c_{n}}-d_{c_{n}\left(s_{n}\right)}^{s_{n}}\right|<1 / n$ on $K_{n}$. (The same is true with $s_{n}$ replaced by any $s$ with $s_{n} \leq s<\omega_{n}$, but we have no particular need for that.) Let $\delta_{n}=d_{c_{n}\left(s_{n}\right)}^{s_{n}}$.

Suppose that the sequence $\left\{b_{c_{n}}\right\}$ converges pointwise to $f$. Then by uniform convergence on each $K_{k}$, we have for any $\epsilon>0$, there is some $N_{\epsilon}^{k}$ such that for any $n \geq N_{\epsilon}^{k}$,

$$
\left|f-b_{c_{n}}\right|<\frac{\epsilon}{2} \quad \text { on } K_{k} .
$$

We also have for all $n>\frac{2}{\epsilon}$,

$$
\left|b_{c_{n}}-\delta_{n}\right|<\frac{\epsilon}{2} \quad \text { on } K_{n} .
$$

Therefore, for all $n>\max \left\{N_{\epsilon}^{k}, \frac{2}{\epsilon}, k\right\}$,

$$
\left|f-\delta_{n}\right|<\epsilon \quad \text { on } K_{k} \text {. }
$$

This shows $\left\{\delta_{n}\right\}$ converges to $f$ uniformly on compact subsets, so the convergence is also pointwise.

Suppose $\left\{\delta_{n}\right\}$ converges pointwise to $f$. Then we proceed similarly: For each $k$, for every $\epsilon>0$, there is some $M_{\epsilon}^{k}$ so that for every $n \geq M_{\epsilon}^{k},\left|f-\delta_{n}\right|<\frac{\epsilon}{2}$ on $K_{k}$. Then for $n>\max \left\{M_{\epsilon}^{k}, \frac{2}{\epsilon}, k\right\},\left|f-b_{c_{n}}\right|<\epsilon$ on $K_{k}$, and $\left\{b_{c_{n}}\right\}$ converges to $f$. 
For future-chronological limits, it works similarly:

Suppose $f \in \hat{L}\left(\left\{b_{c_{n}}\right\}\right)$; that means $f \leq \liminf _{n \rightarrow \infty} b_{c_{n}}$ and $f$ is the only function $g \in \mathcal{I P}(M)$ such that $f \leq g \leq \limsup _{n \rightarrow \infty} b_{c_{n}}$. We use the fact that for any $x \in M$, for some integer $I_{x}$, for all $n \geq I_{x}, x \in K_{n}$.

We know that for all $x \in M$, for all $\epsilon>0$, for some $J_{\epsilon}^{x}$, for all $n \geq J_{\epsilon}^{x}$,

$$
f(x) \leq b_{c_{n}}(x)+\frac{\epsilon}{2} .
$$

We also know for $n \geq \max \left\{I_{x}, \frac{2}{\epsilon}\right\}$,

$$
\left|b_{c_{n}}(x)-\delta_{n}(x)\right|<\frac{\epsilon}{2} .
$$

Then for all $n>\max \left\{I_{x}, J_{\epsilon}^{x}, \frac{2}{\epsilon}\right\}, f(x) \leq \delta_{n}(x)+\epsilon$; thus, $f \leq \liminf \delta_{n}$.

For any $g \leq \limsup \delta_{n}$, we know that for all $x \in M$, for all $\epsilon>0$, there is a subsequence $\sigma$ of the integers such that for all $k$,

$$
g(x) \leq \delta_{\sigma(k)}(x)+\frac{\epsilon}{2} .
$$

Then for $k$ so large that $\sigma(k) \geq \max \left\{I_{x}, \frac{2}{\epsilon}\right\}$,

$$
\left|b_{c_{\sigma(k)}}(x)-\delta_{\sigma(k)}(x)\right|<\frac{\epsilon}{2},
$$

so $g(x) \leq b_{c_{\sigma(k)}}(x)+\epsilon$; thus, $g \leq \lim \sup b_{c_{n}}$. It follows that if $f \leq g \leq \lim \sup \delta_{n}$, then $f \leq g \leq \limsup b_{c_{n}}$, so $g=f$; thus, $f \in \hat{L}\left(\left\{\delta_{n}\right\}\right)$.

Suppose $f \in \hat{L}\left(\left\{\delta_{n}\right\}\right)$. Then we proceed exactly the same way, just exchanging the roles of $\delta_{n}$ and $b_{c_{n}}$, and ending with $f \in \hat{L}\left(\left\{b_{c_{n}}\right\}\right)$.

\section{Asymptotically Ray-Like Curves and Rays}

In a Hadamard manifold, the only Busemann functions are those which are generated by rays; but that may not be the case in a general manifold. An example occurs in a manifold similar to that of the unwrapped grapefruit on a stick of section 2: Instead of just one grapefruit, have an infinite number of them. In other words: $M$ has alternating strips of zero and uniform positive curvature (separated by minuscule regions of negative curvature). If the $k$ th grapefruit, of radius 1 , is centered at $z=4 k$, then the $k$ th flat region is given by $4 k+1<z<4 k+3$. Let $c_{k}$ be a ray in the $k$ th flat region, $c_{k}(t)=((1 / r) t, 4 k+2)$ (where, as before, $r$ is the radius of the stick); then $b_{c_{k}}$ has a simple formula for those points $(\theta, z)$ lying in the flat regions. With $\kappa(z)$ denoting which flat region $(\theta, z)$ lies in (i.e., $4 \kappa+1<z<4 \kappa+3)$, we have

$$
b_{c_{k}}(\theta, z)=r \theta-(1-r)|k-\kappa(z)| \pi .
$$

For points in the round regions, the formula for this Busemann function involves the function $f$ from the example in section 2 ; but we can get a good picture of what's transpiring here without engaging that level of detail. The important point to note here is that for fixed $\theta$, the $k$ th Busemann function peaks for points in the $k$ th flat region, and that is the only way in which these functions differ from one 
another. Thus, $b_{c_{k+1}}+(1-r) \pi$ is identical with $b_{c_{k}}$ for those points with $\kappa \leq k$ and is greater than it for the remainder. In other words, with proper adjusting by additive constants-i.e., using $\bar{c}_{k}=c_{k}^{(1-r) k \pi}$ in place of $c_{k}$, thus generating the Busemann function $b_{\bar{c}_{k}}=b_{c_{k}}+(1-r) k \pi$ - the respective IPs generated by these rays are sandwiched together very much like the IPs in $\mathbb{L}^{2}$ given by $P_{k}=I^{-}(k, k)$. And just as $\bigcup_{k=0}^{\infty} P_{k}$ is an IP in $\mathbb{L}^{2}$, the union of the properly sandwiched IPs generated by $\left\{\bar{c}_{k}\right\}$ in this space is another IP. This is not obvious at first blush; it's easy to calculate what the limiting function $b_{\infty}=\lim _{k \rightarrow \infty} b_{\bar{c}_{k}}$ is:

$$
b_{\infty}(\theta, z)=r \theta+(1-r) \kappa(z) \pi
$$

but it is not immediately clear that this is a Busemann function, i.e., that $\mathrm{P}\left(b_{\infty}\right)$ is an IP. It's clearly not the Busemann function of any geodesic, as those are all known: the functions $b_{c_{k}}$ above and the functions coming from geodesics pitched at an angle to the horizontal (in the flat regions); the geodesics remaining within a round section have infinite Busemann fucnctions. But is there any curve $c$ with $b_{c}=b_{\infty}$ ?

We will form a broken geodesic $c$ by taking minimal geodesic arcs between points $\left\{x_{n}\right\}$, where $x_{n}$ lies in the $n$th flat region: $x_{n}=\left(\theta_{n}, 4 n+2\right)$. Then we must have $c\left(t_{n}\right)=x_{n}$ where $t_{n}=\sum_{i=1}^{n} d\left(x_{i-1}, x_{i}\right)$. This yields

$$
\begin{aligned}
b_{c}(\theta, z)=\kappa(z) \pi & +\lim _{n \rightarrow \infty}\left(\sum_{i=1}^{n} \sqrt{r^{2}\left(\theta_{i}-\theta_{i-1}-\pi\right)^{2}+4}\right. \\
& \left.-\sqrt{r^{2}\left(\theta_{n}-n \pi-(\theta-k \pi)\right)^{2}+\left(2 n-z+z_{0}+2\left(k-k_{0}\right)\right)^{2}}\right) .
\end{aligned}
$$

This is not necessarily finite; but if we have $\left\{\theta_{n}\right\}$ increase sufficiently quickly, it will be. Specifically, we need $\lim _{n \rightarrow \infty} \theta_{n} / n=\infty$ and $\sum_{i=1}^{\infty} 1 /\left(\theta_{i}-\theta_{i-1}-\pi\right)<\infty$ (example: $\left.\theta_{n}=n^{3}\right)$. With that provision, we have

$$
b_{c}(\theta, z)=r\left(\theta-\theta_{0}\right)+(1-r) \kappa(z) \pi+C,
$$

where $C$ is a positive constant bounded above by $\sum_{i=1}^{\infty} 2 /\left(r\left(\theta_{i}-\theta_{i-1}-\pi\right)\right)$. (Reaching this simplification requires use of the identity $\sqrt{a^{2}+b}=a+\frac{b}{\sqrt{a^{2}+b}+a}$.) Thus, $b_{\infty}$ is in the same equivalence class as the Busemann function for $c$.

This section is devoted to exploring just how the Busemann function of an arbitrary asymptotically ray-like curve $c$ - which may not be the Busemann function of any geodesic - is related to the Busemann functions of geodesics, specifically, of rays related to $c$. It is applicable to complete manifolds (though results can be obtained for incomplete manifolds which are appropriately convex).

Our first result says that although the Busemann function from a curve $c \in$ $\mathcal{C}_{0}(M)$ may not come from a geodesic, there is always a geodesic $\gamma$ whose Busemann function is in some sense an approximation of that of $c$, in that $b_{\gamma}$ is bounded above by $b_{c}$ and the two functions are equal along $\gamma$. What is particularly interesting is that if the two are also equal along $c$ then they are equal everywhere. 
Proposition 4.1. Suppose $M$ is complete. Let c be any asymptotically ray-like curve and $x_{0}$ any point in $M$. Then there is an endless unit-speed ray $\gamma$ in $M$, starting at $x_{0}$, such that

(1) $b_{\gamma}$ and $b_{c}$ are equal on $\gamma$, and

(2) $b_{\gamma} \leq b_{c}$ on $M$.

Furthermore, for any sequence $\left\{s_{n}\right\}$ of numbers approaching $\infty$,

(3) if $\lim _{n \rightarrow \infty}\left(b_{\gamma}\left(c\left(s_{n}\right)\right)-b_{c}\left(c\left(s_{n}\right)\right)\right)=0$ (i.e., $b_{\gamma}-b_{c}$ goes to 0 along a cofinal sequence on $c)$, then $b_{\gamma}=b_{c}$ on all of $M$.

Proof. For each $n$, let $\gamma_{n}$ be a unit-speed minimal geodesic segment from $x_{0}$ to $c(n)$. We will parametrize $\gamma_{n}$ on $\left[\alpha, T_{n}\right]$, where $\alpha=b_{c}\left(x_{0}\right)$, so that $T_{n}-\alpha=d\left(x_{0}, c(n)\right)$, or

$$
b_{c}\left(x_{0}\right)=T_{n}-d\left(x_{0}, c(n)\right) .
$$

We also know

$$
\begin{aligned}
b_{c}\left(x_{0}\right) & =\lim _{n \rightarrow \infty}\left(n-d\left(x_{0}, c(n)\right)\right) \\
& =\lim _{n \rightarrow \infty}\left(n-T_{n}+b_{c}\left(x_{0}\right)\right)
\end{aligned}
$$

whence

$$
0=\lim _{n \rightarrow \infty}\left(n-T_{n}\right)
$$

We thus know that $\left\{T_{n}\right\}$ goes out to infinity.

The unit vectors $\left\{\dot{\gamma}_{n}(\alpha)\right\}$ (all at the point $x_{0}$ ) have a vector $v$ which is a limitpoint of that sequence, and we will consider the sequence $\left\{\gamma_{n}\right\}$ replaced by a subsequence with $\left\{\dot{\gamma}_{n}(\alpha)\right\}$ converging to $v$. Let $\gamma:[\alpha, \infty) \rightarrow M$ be the geodesic with $\dot{\gamma}(\alpha)=v$. Since every arc of $\gamma$ (say, on $[\alpha, s]$ ) is the limit of $\operatorname{arcs}$ in $\left\{\gamma_{n}\right\}$ (with $\left.T_{n}>s\right), \gamma$ is a ray.

Fix any number $s>b_{c}\left(x_{0}\right)$. We will show $b_{c}(\gamma(s))=s$; since $\gamma$ is a ray, $b_{\gamma}(\gamma(s))=s$, thus proving (1).

Consider the triangle of points $\gamma_{n}(s), \gamma(s)$, and $c(n)$; note that the distance between the first two, $\delta_{n}^{s}=d\left(\gamma_{n}(s), \gamma(s)\right)$, goes to zero as $n$ goes to infinity (since $\left\{\gamma_{n}\right\}$ approaches $\gamma$ on compact subsets), and the distance between the first and third is $d\left(\gamma_{n}(s), c(n)\right)=T_{n}-s$ (since $\left.c(n)=\gamma_{n}\left(T_{n}\right)\right)$. From the triangle inequality we have $\left|T_{n}-s-d(\gamma(s), c(n))\right| \leq \delta_{n}^{s}$. This gives us $\lim _{n \rightarrow \infty}\left(T_{n}-s-d(\gamma(s), c(n))\right)=0$, or $\lim _{n \rightarrow \infty}\left(T_{n}-d(\gamma(s), c(n))\right)=s$. Since, as shown previously, $\lim _{n \rightarrow \infty}\left(T_{n}-n\right)=0$, this establishes $\lim _{n \rightarrow \infty}(n-d(\gamma(s), c(n)))=s$ or $b_{c}(\gamma(s))=s$, as desired.

Thus we have shown that $b_{\gamma}=b_{c}$ along $\gamma$, i.e, that (1) is true. In particular, we have that $\gamma$ is $\gamma$-asymptotically like $b_{c}$. By Lemma 3.2 (using $f=b_{c}$ ) we conclude that $b_{\gamma} \leq b_{c}$ on $M$, i.e., (2) is true.

Suppose now that $b_{\gamma}-b_{c}$ goes to zero along a cofinal sequence $\left\{s_{k}\right\}$ on $c$; since, by Lemma $3.1, b_{c}(c(s))-s$ goes to zero as $s$ goes to infinity, this implies $b_{\gamma}\left(c\left(s_{k}\right)\right)-s_{k}$ goes to zero. Again employing Lemma 3.2 (this time with $f=b_{\gamma}$ ), we conclude $b_{c} \leq b_{\gamma}$ on $M$. It follows that $b_{\gamma}=b_{c}$ on $M$, i.e., (3) is true.

Although the Busemann function for a curve $c$ may not be the Busemann function for any geodesic, we can none the less approximate it by the Busemann functions of geodesics, in that it is the pointwise limit of such functions. 
Proposition 4.2. Suppose $M$ is complete. Let c be any asymptotically ray-like curve in $M$. Then there is a sequence of rays $\left\{\gamma_{n}\right\}$ such that $b_{c}=\lim _{n \rightarrow \infty} b_{\gamma_{n}}$ (pointwise limit, hence, uniform convergence on compact subsets). Also, for all $n$, $b_{\gamma_{n}} \leq b_{c} ;$ thus, $b_{c}=\sup _{n} b_{\gamma_{n}}$.

Proof. For each $n$, apply Proposition 4.1 to the curve $c$ and the point $c(n)$ to obtain the ray $\gamma_{n}$. We have $b_{\gamma_{n}} \leq b_{c}$ on $M$ and $b_{\gamma_{n}}=b_{c}$ along $\gamma_{n}$. In particular, since $\gamma_{n}$ starts at $c(n)$, we have

$$
b_{\gamma_{n}}(c(n))=b_{c}(c(n)) .
$$

Consider an arbitrary point $x \in M$. Since $b_{\gamma_{n}} \in \mathcal{L}_{1}(M)$, we have

$$
\begin{aligned}
b_{\gamma_{n}}(x) & \geq b_{\gamma_{n}}(c(n))-d(c(n), x) \\
& =b_{c}(c(n))-d(c(n), x) .
\end{aligned}
$$

Note that for all $s, b_{c}(c(s)) \geq s$ : We know $b_{c}(c(s))=\lim _{t \rightarrow \infty}(t-d(c(t), c(s)))$, and $t \mapsto t-d(c(t), c(s))$ is an increasing function. Thus, for all $t, b_{c}(c(s)) \geq$ $t-d(c(t), c(s))) \geq t-|t-s|$; choosing $t \geq s$, we get $b_{c}(c(s)) \geq s$. In particular, we have $b_{c}(c(n)) \geq n$. Thus, from (4.1) plus $b_{c} \geq b_{\gamma_{n}}$ we have

$$
b_{c}(x) \geq b_{\gamma_{n}}(x) \geq n-d(c(n), x) .
$$

Since $b_{c}(x)=\lim _{n \rightarrow \infty}(n-d(c(n), x))$, we end up with

$$
b_{c}(x)=\lim _{n \rightarrow \infty} b_{\gamma_{n}}(x) \text {. }
$$

\section{The Limit-Operator $\hat{L}$ and Quasi-Compactness}

In this section we will present a thorough analysis of the limit-operator $\hat{L}$ defining the future-chronological topology in $V^{+}$. This will include what are likely the most important results of this paper: that the function-space and future-chronological topologies coincide if and only if the latter is Hausdorff, and that $M^{*}$ is always compact in the chronological topology. In fact, the future-chronological topology induces a quasi-compactness on any future-complete chronological set, translating into a very useful compactness feature for spacetimes.

We will begin with a way of characterizing $\hat{L}$ in a general chronological set $X$. Much of what we prove in this section is not restricted to standard static spacetimes, or even to spacetimes generally, but is applicable more widely, such as to spacetimes with boundaries. As in section 2, for simplicity of presentation we will restrict our attention to those chronological sets which are past-regular, i.e., in which the past of every point is indecomposable; as previously mentioned, this includes both spacetimes and future-completions of spacetimes, thus everything considered in this paper.

Let $\left\{A_{n}\right\}$ be a sequence of subsets in a set $X$. We will define $\operatorname{LI}\left(\left\{A_{n}\right\}\right)$ (meaning $\liminf _{n \rightarrow \infty}\left\{A_{n}\right\}$ ) and $\operatorname{LS}\left(\left\{A_{n}\right\}\right)$ (meaning $\limsup _{n \rightarrow \infty}\left\{A_{n}\right\}$ ) as follows:

$$
\begin{gathered}
\operatorname{LI}\left(\left\{A_{n}\right\}\right)=\bigcup_{n=1}^{\infty} \bigcap_{k=n}^{\infty} A_{k}, \\
\operatorname{LS}\left(\left\{A_{n}\right\}\right)=\bigcap_{n=1}^{\infty} \bigcup_{k=n}^{\infty} A_{k} .
\end{gathered}
$$


It is evident that these constructions can also be characterized as

$$
\begin{aligned}
\operatorname{LI}\left(\left\{A_{n}\right\}\right) & =\left\{x \in X \mid x \in A_{n} \text { for all } n \text { sufficiently large }\right\} \\
\operatorname{LS}\left(\left\{A_{n}\right\}\right) & =\left\{x \in X \mid x \in A_{n} \text { for infinitely many } n\right\} .
\end{aligned}
$$

These constructions make for an easy way to express the future-chronological limit-operator $\hat{L}$ in a chronological set:

Proposition 5.1. Let $X$ be a past-regular chronological set, and let $\sigma=\left\{\alpha_{n}\right\}$ be any sequence in $X$. Then for any $\alpha \in X, \alpha \in \hat{L}(\sigma)$ if and only if

(1) $I^{-}(\alpha) \subset \operatorname{LI}\left(\left\{I^{-}\left(\alpha_{n}\right)\right\}\right)$ and

(2) $I^{-}(\alpha)$ is a maximal IP in $\operatorname{LS}\left(\left\{I^{-}\left(\alpha_{n}\right)\right\}\right)$.

In particular, if $\operatorname{LI}\left(\left\{I^{-}\left(\alpha_{n}\right)\right\}\right)=\operatorname{LS}\left(\left\{I^{-}\left(\alpha_{n}\right)\right\}\right)=R$, then $\alpha \in \hat{L}(\sigma)$ if and only if $I^{-}(\alpha)$ is a maximal IP in $R$.

In all the above, one may replace $\operatorname{LI}\left(\left\{I^{-}\left(\alpha_{n}\right)\right\}\right)$ and $\operatorname{LS}\left(\left\{I^{-}\left(\alpha_{n}\right)\right\}\right)$ by, respectively, $I^{-}\left[\operatorname{LI}\left(\left\{I^{-}\left(\alpha_{n}\right)\right\}\right)\right]$ and $I^{-}\left[\operatorname{LS}\left(\left\{I^{-}\left(\alpha_{n}\right)\right\}\right)\right]$.

Proof. Conditions (1) and (2) above are just restatements of the two conditions given in the definition in section 2: Condition (1) in section 2 amounts to saying that for all $\beta \in I^{-}(\alpha), \beta \in I^{-}\left(\alpha_{n}\right)$ for $n$ sufficiently large. Condition (2) in section 2 says that if $P$ is any IP properly containing $I^{-}(\alpha)$, then $P$ contains a point $\beta$ with $\beta \notin I^{-}\left(\alpha_{n}\right)$ for $n$ sufficiently large; that is the same as saying that $P$ is not contained in $\operatorname{LS}\left(\left\{\alpha_{n}\right\}\right)$.

For any set $A$ and any past set $P, P \subset A$ if and only if $P \subset I^{-}[A]$; this allows the replacement in the last statement of the proposition.

Note that $\operatorname{LI}\left(\left\{I^{-}\left(\alpha_{n}\right)\right\}\right)$ and $\operatorname{LS}\left(\left\{I^{-}\left(\alpha_{n}\right)\right\}\right)$ generally are not past sets. For instance, in $\mathbb{L}^{2}$, for each $n$, let $\alpha_{n}$ be the point with $t=0$ and $x=1 / n$; then $\operatorname{LI}\left(\left\{I^{-}\left(\alpha_{n}\right)\right\}\right)=\operatorname{LS}\left(\left\{I^{-}\left(\alpha_{n}\right)\right\}\right)=\{(t, x)|t<-| x \mid$ or $t=-x<0\}$ : a past set plus some of its boundary.

On the other hand, the union of a future chain of IPs is always an IP (this will be important for an application of the proposition above):

Lemma 5.2. Let $\left\{P_{\iota} \mid \iota \in I\right\}$ be a collection of IPs indexed by a well-ordered set I such that for all $\iota$ and $\kappa$ in $I, \iota \leq \kappa$ implies $P_{\iota} \subset P_{\kappa}$. Then $\bigcup_{\iota} P_{\iota}$ is an IP.

Proof. Let $P=\bigcup_{\iota} P_{\iota}$. It is easy to show that $P$ is a past set (i.e., that $I^{-}[P]=P$ ). Then to show $P$ is an IP, we can use the alternate characterization of IPs from Theorem 2 of [H1]: A past set $A$ is an IP if and only if for all $\alpha$ and $\beta$ in $A$, there is some $\gamma \in A$ with $\alpha \ll \gamma$ and $\beta \ll \gamma$. This is easily applied here: If $\alpha$ and $\beta$ are in $P$, then $\alpha \in P_{\iota}$ and $\beta \in P_{\kappa}$ for some $\iota$ and $\kappa$; either $\iota \leq \kappa$ or $\kappa \leq \iota$, and this characterization does the rest.

As a direct consequence of Proposition 5.1, we can characterize a class of sequences which always have future-chronological limits.

Proposition 5.3. Let $X$ be a past-regular, future-complete chronological set and let $\sigma=\left\{\alpha_{n}\right\}$ be a sequence in $X$. If $\operatorname{LI}\left(\left\{I^{-}\left(\alpha_{n}\right)\right\}\right)=\operatorname{LS}\left(\left\{I^{-}\left(\alpha_{n}\right)\right\}\right)=R$ is nonempty, then $\hat{L}(\sigma) \neq \emptyset$. (Alternatively: If $I^{-}\left[\operatorname{LI}\left(\left\{I^{-}\left(\alpha_{n}\right)\right\}\right)\right]=I^{-}\left[\operatorname{LS}\left(\left\{I^{-}\left(\alpha_{n}\right)\right\}\right)\right]$ is non-empty, then the same.)

Furthermore, for any $\alpha \in R, \hat{L}(\sigma)$ contains an element $\beta$ with $I^{-}(\beta) \supset I^{-}(\alpha)$. 
Proof. There is some $\alpha_{0} \in R$. Clearly, for any $\alpha \ll \alpha_{0}, \alpha \in R$ also, i.e., $I^{-}\left(\alpha_{0}\right)$ is an IP contained in $R$. Let $\mathcal{P}$ be the set of all IPs contained in $R$, and consider $\mathcal{P}$ as a partially ordered set under inclusion. We know $\mathcal{P}$ is non-empty. If $\left\{P_{\iota} \mid \iota \in I\right\}$ is any chain in this poset ( $I$ a well-ordered index set with $P_{\iota} \subset P_{\kappa}$ for $\left.\iota \leq \kappa\right)$, then by Lemma 5.2, $Q=\bigcup_{\iota} P_{\iota}$ is an IP; therefore, $Q \in \mathcal{P}$. Thus, every chain in $\mathcal{P}$ has an upper bound; by Zorn's lemma there is a maximal IP $P_{\infty} \in \mathcal{P}$.

Let $c$ be a future chain generating $P_{\infty}$. Since $X$ is future-complete, $c$ has a future-limit $\beta$; then $P_{\infty}=I^{-}(\beta)$. By Proposition 5.1, $\beta \in \hat{L}(\sigma)$.

For any $\alpha \in R$ we can restrict attention to the poset $\mathcal{P}_{\alpha}$ consisting of all IPs in $R$ which contain $I^{-}(\alpha)$. We can thus assure that $P_{\infty}$ contains $I^{-}(\alpha)$.

We can thus specify a class of sequences in a spacetime which have a futurechronological limit (if not in the spacetime itself, then in its future-completion):

Corollary 5.4. Let $U$ be a strongly causal spacetime and let $\sigma=\left\{p_{n}\right\}$ be a sequence of events in $U$. Suppose $\sigma$ has these two properties:

(1) There is some $q_{0}$ in the common past of infinitely many $p_{n}$.

(2) If $q \ll p_{n}$ for infinitely many $n$, then $q \ll p_{n}$ for all $n$ sufficiently large.

Then $\sigma$ has a future-chronological limit $\alpha$ in $U^{+}$with $I^{-}(\alpha) \supset I^{-}\left(q_{0}\right)$.

Proof. Apply Proposition 5.3 to $U^{+}$.

Let us apply Propositions 5.1 and 5.3 to our standard static spacetime $V=$ $\mathbb{L}^{1} \times M$. As in section 2, we will identify $V^{+}$with $\mathcal{I P}(M)$ (with $V$ represented by $\mathcal{D}(M))$.

Proposition 5.5. Let $V=\mathbb{L}^{1} \times M$ be a conformal equivalent of a standard static spacetime. Suppose $\left\{f_{n}\right\}$ is a sequence of elements in $\mathcal{I} \mathcal{P}(M)$ (representing elements of $V^{+}$) such that $\phi=\lim _{n} f_{n}$ exists as a pointwise limit and $\phi \neq-\infty$ (though $\phi=\infty$ is allowed). Then $\left\{f_{n}\right\}$ has a future-chronological limit in $V^{+}$. In particular, for any $x \in M$ and $t<\phi(x), \hat{L}\left(\left\{f_{n}\right\}\right)$ contains an element $f_{\infty} \geq d_{x}^{t}$.

Furthermore, for any $f \in \mathcal{I P}(M), f \in \hat{L}\left(\left\{f_{n}\right\}\right)$ if and only if

(1) $f \leq \phi$ and

(2) for any $g \in \mathcal{I P}(M), f \leq g \leq \phi$ implies $g=f$.

In particular, if $\phi \in \mathcal{I P}(M)$, then that is the only future-chronological limit of the sequence: $\hat{L}\left(\left\{f_{n}\right\}\right)=\{\phi\}$.

Proof. With $\left\{f_{n}\right\}$ converging pointwise to $\phi$, it's easy to show $\mathrm{P}(\phi) \subset \mathrm{LI}\left(\left\{\mathrm{P}\left(f_{n}\right)\right\}\right)$ and $\operatorname{LS}\left(\left\{\mathrm{P}\left(f_{n}\right)\right\}\right) \subset$ closure $(\mathrm{P}(\phi))$; therefore, $\operatorname{LI}\left(\left\{\mathrm{P}\left(f_{n}\right)\right\}\right)$ and $\mathrm{LS}\left(\left\{\mathrm{P}\left(f_{n}\right)\right\}\right)$ differ, at most, by some part of $\partial(\mathrm{P}(\phi))$, i.e., points of the form $(\phi(x), x)$. In any case, the two have the same interior, hence, the same past, so Proposition 5.3 applies with the alternative condition.

Since $\phi \neq-\infty, \operatorname{LI}\left(\left\{\mathrm{P}\left(f_{n}\right)\right\}\right.$ ) is non-empty (for any $x \in M$ and $t<\phi(x)$, $\left.(t, x) \in \operatorname{LI}\left(\left\{\mathrm{P}\left(f_{n}\right)\right\}\right)\right)$. Therefore, we can apply Proposition 5.3 and obtain a futurechronological limit; in particular, for any $t<\phi(x)$, since $(t, x) \in \operatorname{LI}\left(\left\{\mathrm{P}\left(f_{n}\right)\right\}\right)$, there is a future-chronological limit $f_{\infty}$ with $\mathrm{P}\left(f_{\infty}\right) \supset I^{-}((t, x))$, i.e., $f_{\infty} \geq d_{x}^{t}$. Proposition 5.1 yields the remainder.

In case $\phi \in \mathcal{I P}(M)$, then $\phi$ clearly satisfies the conditions on $f$ in (1) and (2), so $\phi \in \hat{L}\left(\left\{f_{n}\right\}\right)$. Furthermore, if $f$ satisfies (1) and (2), then letting $g=\phi$ shows that $f=\phi$. 
It is important to realize that even though a sequence of functions $\left\{f_{n}\right\}$ in $\mathcal{I P}(M)$ has a pointwise limit $\phi, \phi$ itself may not represent any point in $V^{+}$; this was the case for sequences of points in the unwrapped grapefruit of section 2. In fact, when this happens, $V^{+}$is necessarily non-Hausdorff in the future-chronological topology:

Proposition 5.6. Suppose $\left\{f_{n}\right\}$ is a sequence of elements in $\mathcal{I P}(M)$ with a pointwise limit $\phi$. If $\hat{L}\left(\left\{f_{n}\right\}\right)$ has an element $f \neq \phi$, then it has at least two elements.

Proof. We know $\phi \neq-\infty$, for otherwise $\operatorname{LS}\left(\left\{\mathrm{P}\left(f_{n}\right)\right\}\right)$ would be empty, but $f \in$ $\hat{L}\left(\left\{f_{n}\right\}\right)$. Using Proposition 5.5, we know $f \leq \phi$. With $f \neq \phi$, there is some $x_{0} \in M$ and $t_{0} \in \mathbb{R}$ with $f\left(x_{0}\right)<t_{0}<\phi\left(x_{0}\right)$. Note that $d_{x_{0}}^{t_{0}}<\phi$ : For any $x$, $d_{x_{0}}^{t_{0}}(x)=t_{0}-d\left(x, x_{0}\right)<\phi\left(x_{0}\right)-d\left(x, x_{0}\right) \leq \phi(x)$ (since $\phi$ is Lipschitz-1).

Let us define

$$
F=\left\{g \in \mathcal{I P}(M) \mid g \leq \phi \text { and } g\left(x_{0}\right) \geq t_{0}\right\},
$$

considered as a partially ordered set under $\leq$ (as applied to functions). We have $d_{x_{0}}^{t_{0}} \in F$, so it is non-empty. For any chain $\left\{g_{\iota}\right\}$ in $F, g=\sup _{\iota} g_{\iota}$ is in $F$ (as $\mathrm{P}(g)=\bigcup_{\iota} \mathrm{P}\left(g_{\iota}\right)$, Lemma 5.2 shows $\left.g \in \mathcal{I P}(M)\right)$, and it is clearly an upper bound for the chain. Therefore, by Zorn's lemma, $F$ contains a maximal function $g_{\infty}$. For any $h \in \mathcal{I P}(M)$ with $g_{\infty} \leq h \leq \phi$, we have $h \in F$; therefore, $g_{\infty}$ being maximal, we must have $h=g_{\infty}$. Thus, by Proposition 5.5, $g_{\infty} \in \hat{L}\left(\left\{f_{n}\right\}\right)$. But since $g_{\infty}\left(x_{0}\right)>f\left(x_{0}\right)$, we know $g_{\infty} \neq f$.

It is less easy to express this idea in other contexts. For any strongly causal spacetime $U$ we could say that if $\sigma=\left\{p_{n}\right\}$ is a sequence of events with $\operatorname{LI}\left(\left\{I^{-}\left(p_{n}\right)\right\}\right)=$ $\operatorname{LS}\left(\left\{I^{-}\left(p_{n}\right)\right\}\right)=R$ but with $\hat{L}(\sigma)$ containing an element $P$ with past different from interior $(R)$, then $\hat{L}(\sigma)$ has at least two elements $(\hat{L}$ being understood to take values in $\left.U^{+}\right)$. For a past-regular, future-complete chronological set $X$, if $\sigma=\left\{\alpha_{n}\right\}$ is a sequence with $\operatorname{LI}\left(\left\{I^{-}\left(\alpha_{n}\right)\right\}\right)=\operatorname{LS}\left(\left\{I^{-}\left(\alpha_{n}\right)\right\}\right)=R$ but with $\hat{L}(\sigma)$ containing a point $\alpha$ with $I^{-}(\alpha) \neq I^{-}[R]$, then $\hat{L}(\sigma)$ contains at least two points. The proofs are essentially identical to that of Proposition 5.6. (For the latter, suppose $\beta_{1} \ll \beta_{2} \in R$ with $\beta_{1} \notin I^{-}(\alpha)$, and let $F=\left\{\operatorname{IPs} P\right.$ in $\left.X \mid I^{-}(\gamma) \subset P \subset R\right\}$ for some $\gamma$ with $\beta_{1} \ll \gamma \ll \beta_{2} ; F$ has a maximal element $I^{-}(\delta)$, and $\delta \neq \alpha$.)

Proposition 5.3 leads to a characterization of the behavior of $\hat{L}$ in non-Hausdorff situations exactly reflective of what happens in the example space $\tilde{G}$ of section 2 (the unwrapped grapefruit on a stick), at least in the simple context of the lim inf and limsup of the pasts of the sequence being the same. Recall that in $\tilde{G}$, the sequence of points $\sigma=\left\{\left(t_{n}, x_{n}\right)\right\}$ for $t_{n}=r n+(1-r) \pi / 2$ and $x_{n}=\left(n, z_{0}\right)$ (with any choice of $\left.\left|z_{0}\right|<r^{\prime}\right)$ has two elements in its future-chronological limit, $\hat{L}(\sigma)=\left\{b^{+}, b^{-}\right\}$ (in the notation of section $2, b^{ \pm}=b_{c^{ \pm}} \pm f\left(z_{0}\right)$ ). Let $\delta_{z_{0}}$ be the function which gives the boundary of the past of this limit set, i.e., $I^{-}[\hat{L}(\sigma)]=\mathrm{P}\left(\delta_{z_{0}}\right)$; this is calculated from the pasts of the points of the sequence as $\delta_{z_{0}}=\lim d_{x_{n}}^{t_{n}}$. Then we also know that $\delta_{z_{0}}=\max \left\{b^{+}, b^{-}\right\}$. In other words: The multiple elements of the future-chronological limit of the sequence have a combined past which is the limit (i.e., both the LI and LS) of the pasts of the sequence. This is true very generally.

Proposition 5.7. Let $X$ be a past-regular, future-complete chronological set. Let $\sigma$ be any sequence of points in $X$ for which $\operatorname{LI}\left(\left\{I^{-}(\sigma(n))\right\}\right)=\operatorname{LS}\left(\left\{I^{-}(\sigma(n))\right\}\right)=$ $R \neq \emptyset$. Then $I^{-}[\hat{L}(\sigma)]=I^{-}[R]$.

Proof. By Proposition 5.1, $\alpha \in \hat{L}(\sigma)$ if and only if $I^{-}(\alpha)$ is a maximal IP in $R$. 
Suppose $\gamma \in I^{-}[\hat{L}(\sigma)]$, i.e., $\gamma \ll \alpha$ for some $\alpha \in \hat{L}(\sigma)$; there is some $\beta$ with $\gamma \ll \beta \ll \alpha$. Then $I^{-}(\alpha) \subset R$, so $\beta \in R$; then $\gamma \in I^{-}[R]$.

Suppose $\gamma \in I^{-}[R]$, i.e., $\gamma \ll \alpha$ for some $\alpha \in \operatorname{LI}\left(\left\{I^{-}(\sigma(n))\right\}\right)$. By Proposition 5.3, $\hat{L}(\sigma)$ contains an element $\beta$ with $I^{-}(\beta) \supset I^{-}(\alpha)$. Then $\gamma \in I^{-}(\beta)$, so $\gamma \in$ $I^{-}[\hat{L}(\sigma)]$.

Let's apply this to a spacetime:

Corollary 5.8. Let $U$ be a strongly causal spacetime. Suppose $\sigma$ is a sequence of events in $U$ for which any event observed by infinitely many of the elements of $\sigma$ is observed by almost all of them (i.e., all but finitely many of them); let $R$ denote this set of observed events, and assume this is non-empty. Then the past of $\hat{L}(\sigma)$ is the past of $R$.

In a standard static spacetime:

Corollary 5.9. Let $V=\mathbb{L}^{1} \times M$ be a conformal equivalent of a standard static spacetime. Suppose $\left\{f_{n}\right\}$ is a sequence of elements in $\mathcal{I P}(M)$ with a pointwise limit $\phi \neq-\infty$. Then $\phi=\sup \hat{L}\left(\left\{f_{n}\right\}\right)$; in particular, if $\hat{L}\left(\left\{f_{n}\right\}\right)$ is finite in size, $\hat{L}\left(\left\{f_{n}\right\}\right)=\left\{g_{1}, \ldots, g_{m}\right\}$, then $\phi=\max _{i \leq m} g_{i}$.

Proof. The sets $\operatorname{LI}\left(\left\{\mathrm{P}\left(f_{n}\right)\right\}\right)$ and $\operatorname{LS}\left(\left\{\mathrm{P}\left(f_{n}\right)\right\}\right)$ have the same boundary precisely when $\left\{f_{n}\right\}$ has a pointwise limit $\phi$, and that boundary is given by $\phi$. Thus, $\mathrm{P}(\phi)$ is the interior-i.e., the past - of $R$ in Corollary 5.8. For any collection $Q$ of functions in $\mathcal{I P}(M)$, the boundary of $I^{-}[Q]$ is given by $\sup _{f \in Q} f$. Thus, Corollary 5.8 identifies $\phi$ as $\sup \hat{L}\left(\left\{f_{n}\right\}\right)$.

The key to the main compactness result is a very minor extension of Proposition 5.3: We may restrict attention to intersection with a countable dense set. We are speaking here of a set $D$ which is chronologically dense, i.e., for any $x \ll y$, there is an element $d \in D$ with $x \ll d \ll y$. The existence of a countable, chronologically dense subset is one of the axioms of a chronological set (see [H1]); it is what makes it possible to do almost as much in such a general setting as one can do in a spacetime.

Lemma 5.10. Let $X$ be a past-regular, future-complete chronological set, and let $\sigma=\left\{\alpha_{n}\right\}$ be any sequence in $X$. Let $D$ be any chronologically dense subset of $X$. If $\operatorname{LI}\left(\left\{I^{-}\left(\alpha_{n}\right)\right\}\right) \cap D=\operatorname{LS}\left(\left\{I^{-}\left(\alpha_{n}\right)\right\}\right) \cap D \neq \emptyset$, then $\hat{L}(\sigma) \neq \emptyset$; in particular, for any $\alpha \in \operatorname{LI}\left(\left\{I^{-}\left(\alpha_{n}\right)\right\}\right), \hat{L}(\sigma)$ contains an element $\beta$ with $I^{-}(\beta) \supset I^{-}(\alpha)$.

Proof. Let $I=\operatorname{LI}\left(\left\{I^{-}\left(\alpha_{n}\right)\right\}\right)$ and $S=\operatorname{LS}\left(\left\{I^{-}\left(\alpha_{n}\right)\right\}\right)$; we have $\alpha \in I$. As in the proof of Proposition 5.3, we can demonstrate the existence of an IP $P_{\infty}$, lying in $I$ and containing $I^{-}(\alpha)$, which is maximal for those properties (let $\mathcal{P}$ be the collection of such IPs; invoking Lemma 5.2, we see every inclusion-chain in $\mathcal{P}$ has an upper bound); this also gives us that $P_{\infty}$ is a maximal IP in $I$. There is some $\beta \in X$ with $I^{-}(\beta)=P_{\infty}$. All we need to show is that $P_{\infty}$ is maximal in $S$, for then Proposition 5.1 gives us $\beta \in \hat{L}(\sigma)$.

Suppose there were some $\gamma \in X$ with $I^{-}(\beta) \subsetneq I^{-}(\gamma) \subset S$. Since $P_{\infty}$ is maximal in $I$, we have $I^{-}(\gamma) \not \subset I$; thus, there is some $\gamma^{\prime} \ll \gamma$ with $\gamma^{\prime} \notin I$. There is some element $\delta \in D$ with $\gamma^{\prime} \ll \delta \ll \gamma$; then $\delta \notin I$ also. That means $\delta \notin I \cap D$, so $\delta \notin S \cap D$ also; on the other hand, $\delta \in I^{-}(\gamma)$, so $\delta$ must be in $S$.

With this lemma in hand, we can use a diagonal argument to establish our basic quasi-compactness result. 
Theorem 5.11. Let $X$ be a past-regular, future-complete chronological set. Let $\sigma$ be any sequence of points in $X$ for which $\operatorname{LS}\left(\left\{I^{-}(\sigma(n))\right\}\right)$ has a non-empty past. Then there is a subsequence $\sigma^{\infty} \subset \sigma$ with $\hat{L}\left(\sigma^{\infty}\right) \neq \emptyset$; in particular, for any $\alpha \in \operatorname{LI}\left(\left\{I^{-}(\sigma(n))\right\}\right), \hat{L}\left(\sigma^{\infty}\right)$ contains an element $\beta$ with $I^{-}(\beta) \supset I^{-}(\alpha)$.

Proof. Let $D=\left\{d_{n}\right\}$ be a countable, chronologically dense set in $X$. We will construct a sequence of nested subsequences of the positive integers, $\cdots \subset \tau^{n} \subset \tau^{n-1} \subset$ $\cdots \subset \tau^{1} \subset \tau^{0}=(1,2,3, \ldots)$. First some notation: For any sequence $\bar{\sigma}$ of points in $X$, let $\operatorname{LS}_{I^{-}}\{\bar{\sigma}\}=\operatorname{LS}\left(\left\{I^{-}(\bar{\sigma}(n))\right\}\right)$; for any subsequence $\tau$ of $(1,2,3, \ldots)$, let $\sigma \circ \tau$ denote the sequence of points $(\sigma(\tau(1)), \sigma(\tau(2)), \ldots)$ and let $\mathrm{S}_{\sigma}[\tau]=\operatorname{LS}_{I^{-}}\{\sigma \circ \tau\}$. Similarly for $\operatorname{LI}_{I^{-}}\{\bar{\sigma}\}$ and $\mathrm{I}_{\sigma}[\tau]$. Note that for $\tau^{\prime} \subset \tau$, we have $\mathrm{S}_{\sigma}\left[\tau^{\prime}\right] \subset \mathrm{S}_{\sigma}[\tau]$. Also, $\mathrm{S}_{\sigma}\left[\tau^{0}\right]=\mathrm{LS}_{I^{-}}\{\sigma\}$.

Suppose we have defined $\tau^{n-1}$. Consider the element $d_{n}$ : If $d_{n} \notin \mathrm{S}_{\sigma}\left[\tau^{n-1}\right]$-i.e., if $d_{n} \ll \sigma\left(\tau^{n-1}(k)\right)$ for only finitely many $k$-then let $\tau^{n}=\tau^{n-1}$. If, however, $d_{n} \in \mathrm{S}_{\sigma}\left[\tau^{n-1}\right]$, then let $\tau^{n}$ be that subsequence of $\tau^{n-1}$ containing all numbers $k$ such that $d_{n} \ll \sigma\left(\tau^{n-1}(k)\right.$ ) (which occurs for infinitely many $k$ ). Note that this implies that if $d_{n} \in \mathrm{S}_{\sigma}\left[\tau^{n-1}\right]$ then for all $k, d_{n} \ll \sigma\left(\tau^{n}(k)\right)$ (a stronger statement than $\left.d_{n} \in \mathrm{I}_{\sigma}\left[\tau^{n}\right]\right)$.

Having defined all the subsequences $\left\{\tau^{n}\right\}$, we have, for all $k \leq j, \mathrm{~S}_{\sigma}\left[\tau^{k}\right] \supset \mathrm{S}_{\sigma}\left[\tau^{j}\right]$. Therefore, for all $n$, if for any $j \geq n-1, d_{n} \in \mathrm{S}_{\sigma}\left[\tau^{j}\right]$ — that is to say, $d_{n}$ is in the past of infinitely many of the elements of $\tau^{j}$ - then $d_{n}$ is also in $S_{\sigma}\left[\tau^{n-1}\right]$ and, consequently, for all $m \geq n$ and for all $k, d_{n} \ll \sigma\left(\tau^{m}(k)\right)$. In summary:

$$
\begin{gathered}
d_{n} \in \mathrm{S}_{\sigma}\left[\tau^{j}\right] \text { for some } j \geq n-1 \Longrightarrow \\
d_{n} \ll \sigma\left(\tau^{m}(k)\right) \text { for all } m \geq n \text { and for all } k .
\end{gathered}
$$

Now let $\tau^{\infty}$ be the sequence defined by $\tau^{\infty}(k)=\tau^{k}(k)$. Consider any $d_{n} \in$ $\mathrm{S}_{\sigma}\left[\tau^{\infty}\right]$ : For infinitely many $k, d_{n} \ll \sigma\left(\tau^{k}(k)\right)$, i.e., there is a strictly increasing sequence of integers $\left\{k_{i}\right\}$ with $d_{n} \ll \sigma\left(\tau^{k_{i}}\left(k_{i}\right)\right)$ for all $i$. Pick some $j$ with $k_{j} \geq n-1$. For all $i \geq j$, we have $\tau^{k_{i}}$ is a subsequence of $\tau^{k_{j}}$, so for some $m_{i}, \tau^{k_{i}}\left(k_{i}\right)=\tau^{k_{j}}\left(m_{i}\right)$. Then $d_{n} \ll \sigma\left(\tau^{k_{j}}\left(m_{i}\right)\right)$ for all $i \geq j$, an infinite collection $\left\{m_{i}\right\}$. Thus, $d_{n} \in \mathrm{S}_{\sigma}\left[\tau^{k_{j}}\right]$. It follows that for all $m \geq n$ and all $k, d_{n} \ll \sigma\left(\tau^{m}(k)\right)$. In particular, we have $d_{n} \ll \sigma\left(\tau^{k}(k)\right)$ for all $k \geq n$, which gives us $d_{n} \in \mathrm{I}_{\sigma}\left[\tau^{\infty}\right]$. Therefore, $\mathrm{I}_{\sigma}\left[\tau^{\infty}\right] \cap D=$ $\mathrm{S}_{\sigma}\left[\tau^{\infty}\right] \cap D$.

Let $\sigma^{\infty}=\sigma \circ \tau^{\infty}$. We have $\operatorname{LI}_{I^{-}}\left\{\sigma^{\infty}\right\} \cap D=\operatorname{LS}_{I^{-}}\left\{\sigma^{\infty}\right\} \cap D$; we need to know this is non-empty. We know $\operatorname{LS}_{I^{-}}\{\sigma\}$ contains some element $\gamma$ with some $\gamma^{\prime} \ll \gamma$. There is some element $\delta$ of $D$ with $\gamma^{\prime} \ll \delta \ll \gamma$. All of $I^{-}(\gamma)$ lies in $\operatorname{LS}_{I^{-}}\{\sigma\}$, so $\delta \in \operatorname{LS}_{I^{-}}\{\sigma\}$, i.e., $\operatorname{LS}_{I^{-}}\{\sigma\} \cap D$ is non-empty. Let $n_{0}=\min \left\{n \mid d_{n} \in \operatorname{LS}_{I^{-}}\{\sigma\}\right\}$. Then for all $i<n_{0}, \tau^{i}=\tau^{0}$, and $d_{n_{0}} \in I^{-}\left(\left(\sigma \circ \tau^{n_{0}}\right)(k)\right)$ for all $k$. For all larger $n, \sigma \circ \tau^{n}$ is a subsequence of $\sigma \circ \tau^{n_{0}}$, so $d_{n_{0}}$ is also in every $I^{-}\left(\left(\sigma \circ \tau^{n}\right)(k)\right)$. In particular, $d_{n_{0}} \in I^{-}\left(\left(\sigma \circ \tau^{n}\right)(n)\right)$ for all $n \geq n_{0}$; it follows that $d_{n_{0}} \in \operatorname{LS}_{I^{-}}\left\{\sigma \circ \tau^{\infty}\right\}$. Therefore, $\operatorname{LS}_{I^{-}}\left\{\sigma^{\infty}\right\} \cap D$ is non-empty.

Lemma 5.10 now applies to $\sigma^{\infty}$; this immediately gives us that $\hat{L}\left(\sigma^{\infty}\right)$ is nonempty. Now consider any $\alpha \in \mathrm{LI}_{I^{-}}\{\sigma\}$; since $\sigma^{\infty}$ is a subsequence of $\sigma$, we also have $\alpha \in \mathrm{LI}_{I^{-}}\left\{\sigma^{\infty}\right\}$. Therefore, Lemma 5.10 gives us an element $\beta \in \hat{L}\left(\sigma^{\infty}\right)$ with $I^{-}(\beta) \supset I^{-}(\alpha)$.

This has immediate application to spacetimes: 
Corollary 5.12. Let $U$ be a strongly causal spacetime, and let $\sigma$ be any sequence of events in $U$. Suppose there is an event in $U$ which is observed by an infinite number of the elements of $\sigma$; then there is a subsequence $\sigma^{\infty} \subset \sigma$ with $\hat{L}\left(\sigma^{\infty}\right) \neq \emptyset$ (where $\hat{L}$ takes values possibly in $\partial^{+}(U)$ ). Moreover, if there is some event $x$ observed by all but finitely many of the elements of $\sigma$, then $\hat{L}\left(\sigma^{\infty}\right)$ contains an element $\beta$ whose past contains the past of $x$.

In other words: A sequence $\sigma$ of events contains a subsequence $\sigma^{\infty}$ with a futurechronological limit if and only if some event in $U$ is in the common past of some subsequence of $\sigma$; and the combined observations of all the future-chronological limits of $\sigma^{\infty}$ include anything observed by almost all the elements of $\sigma$.

Proof. Just apply Theorem 5.11 to $U^{+}$.

Specializing to standard static spacetimes, we have this:

Corollary 5.13. Let $V=\mathbb{L}^{1} \times M$ be a conformal equivalent of a standard static spacetime, and let $\left\{f_{n}\right\}$ be a sequence of elements in $\mathcal{I P}(M)$; let $f^{+}=\lim \sup f_{n}$ and $f^{-}=\liminf f_{n}$. If $f^{+} \neq-\infty$, then there is a subsequence $\left\{f_{n_{k}}\right\}$ with a future-chronological limit in $V^{+}$; moreover, this sequence has the property that for any $x \in M$ and $t<f^{-}(x)$, there is a future-chronological limit $f_{\infty}$ of $\left\{f_{n_{k}}\right\}$ with $f_{\infty} \geq d_{x}^{t}$. That future-chronological limit cannot be $i^{+}$unless $f^{+}=\infty$, which can happen only when there is a subsequence of the events represented by $\left\{f_{n}\right\}$ with no events in its common future. Furthermore, the subsequence $\left\{f_{n_{k}}\right\}$ has a pointwise limit.

Proof. First note that $f^{+}$is either everywhere finite or everywhere infinite (and the same for $\left.f^{-}\right)$. Note also that $\mathrm{P}\left(f^{+}\right)$is the interior of $\operatorname{LS}\left(\left\{\mathrm{P}\left(f_{n}\right)\right\}\right)$ and $\mathrm{P}\left(f^{-}\right)$the interior of $\operatorname{LI}\left(\left\{\mathrm{P}\left(f_{n}\right)\right\}\right)$. Then Theorem 5.11 applies to $V^{+}$.

The statement about $i^{+}$follows from noting that $f_{\infty} \leq f^{+}$, as $\mathrm{P}\left(f_{\infty}\right)$ must lie inside $\operatorname{LS}\left(\left\{\mathrm{P}\left(f_{n}\right)\right\}\right)$.

The final statement follows from noting that the subsequence created in Theorem 5.11 has $\operatorname{LI}\left(\left\{\mathrm{P}\left(f_{n_{k}}\right)\right\}\right) \cap D=\operatorname{LS}\left(\left\{\mathrm{P}\left(f_{n_{k}}\right)\right\}\right) \cap D$ for a countable dense set $D$ (in $V$, chronologically dense is the same as topologically dense). Let $D=\left\{\left(t_{m}, x_{m}\right)\right\}$. Consider the sets

$$
\begin{aligned}
& A=\left\{m \mid \text { for infinitely many } k, t_{m}<f_{n_{k}}\left(x_{m}\right)\right\} \text { and } \\
& B=\left\{m \mid \text { for almost all } k, t_{m}<f_{n_{k}}\left(x_{m}\right)\right\} .
\end{aligned}
$$

Define $\bar{f}^{+}=\limsup f_{n_{k}}$ and $\bar{f}^{-}=\liminf f_{n_{k}}$, and let

$$
\begin{aligned}
& A^{+}=\left\{m \mid t_{m}<\bar{f}^{+}\left(x_{m}\right)\right\} \text { and } \\
& B^{-}=\left\{m \mid t_{m} \leq \bar{f}^{-}\left(x_{m}\right)\right\} .
\end{aligned}
$$

Note that $A^{+} \subset A$ and $B \subset B^{-}$. The information from Theorem 5.11 is that $A=B$. It follows that $A^{+} \subset B^{-}$, i.e.,

$$
\text { for all } m, t_{m}<\bar{f}^{+}\left(x_{m}\right) \text { implies } t_{m} \leq \bar{f}^{-}\left(x_{m}\right) \text {. }
$$

We know $\bar{f}^{+}$and $\bar{f}^{-}$are Lipschitz-1, hence, continuous; so if they do not coincide, then there is some open set $U$ and some numbers $t^{-}<t^{+}$such than on $U, \bar{f}^{-}<t^{-}$ 
and $t^{+}<\bar{f}^{+}$. There are infinitely many $m$ (though only one is needed) such that $\left(t_{m}, x_{m}\right) \in\left(t^{-}, t^{+}\right) \times U$. For each such $m, \bar{f}^{-}\left(x_{m}\right)<t^{-}<t_{m}<t^{+}<\bar{f}^{+}\left(x_{m}\right)$, contradicting the implication displayed above. It follows that $\bar{f}^{-}=\bar{f}^{+}$, which means that $\left\{f_{n_{k}}\right\}$ converges pointwise.

While Corollary 5.13 provides only a quasi-compactness for $V^{+}$(sequences have convergent subsequences only if they don't converge to $-\infty$ ), it is good enough to provide full compactness for $M^{*}$ :

Theorem 5.14. Let $M$ be any Riemannian manifold. Then $M^{*}$ is compact in the chronological topology.

Proof. We represent $M^{*}$ as $V_{0}^{+} / \mathbb{R}\left(V=\mathbb{L}^{1} \times M\right)$, which we treat as $\mathcal{I} \mathcal{P}_{0}(M) / \mathbb{R}$. Let $\sigma=\left\{\left[f_{n}\right]\right\}$ be any sequence in $\mathcal{I P}_{0}(M) / \mathbb{R}$. Pick a point $x_{0} \in M$. For each $n$, let $\bar{f}_{n}=f_{n}-f_{n}\left(x_{0}\right)$; then $\left[\bar{f}_{n}\right]=\left[f_{n}\right]$, so these functions represent the same sequence $\sigma$ in $M^{*}$. By Corollary 5.13, there is a subsequence $\left\{\bar{f}_{n_{k}}\right\}$ with a future-chronological limit $\bar{f}_{\infty}$ in $V_{0}^{+}\left(\left\{\bar{f}_{n}\left(x_{0}\right)\right\}\right.$ being obviously bounded below $)$. Since $\bar{f}_{\infty}$ is a limit of the sequence $\left\{\bar{f}_{n_{k}}\right\}$ in the future-chronological topology on $V_{0}^{+},\left[\bar{f}_{\infty}\right]$ is a limit of the sequence $\left\{\left[\bar{f}_{n_{k}}\right]\right\}=\left\{\left[f_{n_{k}}\right]\right\}$ in the chronological topology on $M^{*}$. (Note that we are using the fact that $\pi: V_{0}^{+} \rightarrow M^{*}$ is continuous in the quotient topology; we are not assuming that $e_{x_{0}}: M^{*} \rightarrow V_{0}^{+}$is continuous.)

Since every sequence in $M^{*}$ has a subsequence with a limit, it is compact.

(This result in purely Riemannian geometry can be established without recourse to chronological sets by proving a result for separable metric spaces: Any sequence of Lipschitz-1 functions which is bounded below at one point has a pointwiseconvergent subsequence with convergence to $\infty$ being allowed. A diagonal procedure is used, employing a countable dense set in the metric space.)

Corollary 5.13 also establishes that it is precisely the non-Hausdorff character of the future-chronological topology that distinguishes it from the function-space topology. Note that it is the behavior of $\hat{L}$-whether or not, for a sequence $\sigma$ in $V^{+}, \hat{L}(\sigma)$ can have more than one element-that determines whether or not the future-chronological topology is the same as the function-space topology: For if $\hat{L}(\sigma)$ never has more than one element, then, by Proposition 5.6, it is the same as the pointwise limit.

Theorem 5.15. Let $V$ be a standard static spacetime. The future-chronological topology on $V^{+}$is identical to the function-space topology if and only if the former is Hausdorff.

Proof. The compact-open topology on a real-valued function space is always Hausdorff (assuming points are closed in the domain space): If $f \neq g$, then there is some $x$ in the domain with, say, $f(x)<g(x)$. Pick a number $a$ with $f(x)<a<g(x)$. Then $\{h \mid h[\{x\}] \subset(-\infty, a)\}$ and $\{h \mid h[\{x\}] \subset(a, \infty)\}$ are disjoint neighborhoods of $f$ and $g$ respectively.

Assume that the future-chronological topology on $V^{+}$is Hausdorff. We will show that any sequence $\left\{f_{n}\right\}$ in $\mathcal{I P}(M)\left(V=\mathbb{L}^{1} \times M\right)$ can have only the pointwise limit of $\left\{f_{n}\right\}$ as a future-chronological limit.

Suppose we have $f \in \hat{L}\left(\left\{f_{n}\right\}\right)$. Let $f^{-}=\liminf f_{n}$ and $f^{+}=\limsup f_{n}$. We first show $f^{-}=f^{+}$:

Suppose the two differ on some point $x$. Let $\left\{f_{m}^{\prime}\right\}$ be a subsequence of $\left\{f_{n}\right\}$ with $\left\{f_{m}^{\prime}(x)\right\}$ converging to $f^{+}(x)$, and let $f^{\prime-}=\liminf f_{m}^{\prime}$. As $f \leq f^{-}$, we know 
$\left\{f_{m}^{\prime}(x)\right\}$ is bounded below by $f(x)$. Pick some $t$ with $f^{-}(x)<t<f^{+}(x)$; note that $t<f^{\prime-}(x)$. Then by Corollary 5.13, there is a subsequence $\left\{f_{m_{k}}^{\prime}\right\}$ with a future-chronological limit $f_{\infty}^{\prime} \geq d_{x}^{t}$. Then

$$
f(x) \leq f^{-}(x)<t=d_{x}^{t}(x) \leq f_{\infty}^{\prime}(x) .
$$

It follows that $f \neq f_{\infty}^{\prime}$. Then, since $\hat{L}\left(\left\{f_{n}\right\}\right) \subset \hat{L}\left(\left\{f_{m_{k}}^{\prime}\right\}\right)$, we have $f$ and $f_{\infty}^{\prime}$ as distinct elements of $\hat{L}\left(\left\{f_{m_{k}}^{\prime}\right\}\right)$, contradicting Hausdorffness.

Therefore, $\left\{f_{n}\right\}$ converges pointwise to some $\phi$ (possibly $\infty$ ). Then, by Proposition 5.6, $\phi=f$ must be the only element of $\hat{L}\left(\left\{f_{n}\right\}\right)$, if $V^{+}$is Hausdorff in the future-chronological topology.

We have shown that under the assumption of Hausdorffness on the futurechronological topology, the only future-chronological limits are also function-space limits; it follows that the two topologies are identical.

\section{When the FunCtion-Space And Future-Chronological Topologies Coincide}

We conclude this paper with an examination of standard static spacetimes $\mathbb{L}^{1} \times M$ for which it can be shown that the future-chronological topology on the future causal boundary is as nice as can be desired: a cone on the Busemann boundary, with only geodesics being needed for Busemann functions. We will show that this includes a large class of spacetimes encompassing various classical examples, such as external Schwarzschild or parts of Schwarzschild-de Sitter or Reissner-Nordström, addressed in a discussion subsection.

We need first a couple of definitions:

Divergence of a sequence of points. Let $\sigma$ be a sequence of points in $M$. Then $\sigma$ is divergent if there is no convergent subsequence (i.e., $\sigma^{\prime} \subset \sigma$ such that $\lim _{n \rightarrow \infty} \sigma^{\prime}(n)$ exists in $\left.M\right)$.

Convergence of a sequence of geodesic segments. Suppose $\left\{\gamma_{n}\right\}$ is a sequence of unit-speed geodesic segments in $M, \gamma_{n}$ defined on $\left[0, L_{n}\right]$. We will say that $\left\{\gamma_{n}\right\}$ converges to the unit speed geodesic $\gamma_{\infty}$, defined on $[0, \omega)$, if

(1) $\left\{\dot{\gamma}_{n}(0)\right\}$ converges to $\dot{\gamma}_{\infty}(0)$,

(2) $\omega=\lim _{n \rightarrow \infty}\left\{L_{n}\right\}$, and

(3) $[0, \omega)$ is the maximal domain for $\gamma_{\infty}$.

Note that these three conditions imply that for all $t<\omega,\left\{\gamma_{n}(t)\right\}$ (defined for $n$ sufficiently large) converges to $\gamma_{\infty}(t)$.

The point of this is to define convergence of geodesics even in a non-complete manifold, where geodesics of maximal length cannot be assumed to be infinite in length. Since a spacetime of interest is typically only conformal to a standard static spacetime, and the conformal factor may easily result in the Riemannian factor being rendered non-complete, we must retain an interest in non-complete manifolds.

The following proposition is a condition that allows us to conclude that the Busemann boundary has the simplest of forms: It has the function-space topology, and it uses only geodesics - and only a restricted class of limit-geodesics, at that. It appears to be an unnaturally formidable condition; but Theorem 6.2 will show that is not the case, that it applies to a substantial class of manifolds. 
Proposition 6.1. Suppose distances in $M$ are realized by geodesics and that $M$ obeys the following property:

For every divergent sequence $\left\{x_{n}\right\}$ in $M$, for some subsequence of that sequence (which we again call $\left\{x_{n}\right\}$ ), for some point $z$, for some choice of minimal unit-speed geodesic segments $\gamma_{n}$ from $z$ to $x_{n}$ (with $\gamma_{n}(0)=z$ ), the geodesic segments converge to a geodesic $\gamma_{\infty}:[0, \omega) \rightarrow M$ such that for all $x \in M$,

$$
\lim _{t \rightarrow \omega}\left(d\left(z, \gamma_{\infty}(t)\right)-d\left(x, \gamma_{\infty}(t)\right)\right)=\lim _{n \rightarrow \infty}\left(d\left(z, x_{n}\right)-d\left(x, x_{n}\right)\right)
$$

(wherein $\infty$ is admissable as a limit); or, more generally, for some sequence $\left\{t_{n}\right\}$ approaching $\omega$, for all $x \in M$,

$$
\lim _{n \rightarrow \infty}\left(d\left(z, \gamma_{\infty}\left(t_{n}\right)\right)-d\left(x, \gamma_{\infty}\left(t_{n}\right)\right)\right)=\lim _{n \rightarrow \infty}\left(d\left(z, x_{n}\right)-d\left(x, x_{n}\right)\right) .
$$

(The left side of either $\left(^{*}\right)$ or $\left({ }^{*}\right)$ always exists with $\infty$ admitted as a value; these hypotheses are conditions upon the right sides.)

Then

(1) the chronological topology on the Busemann boundary of $M, \partial_{B}(M)$, is the same as the function-space topology, and

(2) only geodesics need be considered for $\partial_{B}(M)$; moreover, only geodesics of the form $\gamma_{\infty}$.

Thus, the future causal boundary for $V=\mathbb{L}^{1} \times M$ (in the future-chronological topology) is a cone on $\partial_{B}(M)$, and $\partial_{B}(M)$ may be identified with equivalence classes of Busemann functions of geodesics of the form $\gamma_{\infty}$ above.

Proof. By Theorem 5.15, to show the chronological topology the same as the function-space topology, it suffices to show the former is Hausdorff. By Proposition 5.5 , to show Hausdorffness, it suffices to show that for any sequence of functions $\left\{f_{n}\right\}$ in $\mathcal{I P}(M)$ for which there is a future-chronological limit, there is a subsequence with a pointwise limit $\phi$ such that $\phi \in \mathcal{I P}(M)$ also. By Corollary 5.13 (last statement), we may restrict ourselves to consideration of sequences which have a pointwise limit. By Lemma 3.3, it is sufficient to consider the functions $f_{n}$ as being of the form $a_{n}-d\left(x_{n},-\right)$ for some numbers $\left\{a_{n}\right\}$ and points $\left\{x_{n}\right\}$.

So let us consider a sequence of functions $\left\{d_{n}\right\}$ with $d_{n}=a_{n}-d\left(x_{n},-\right)$ such that $\left\{d_{n}\right\}$ has the pointwise limit $\phi$; we can assume $\phi$ is finite, since $\infty$ is always in $\mathcal{I P}(M)$. Let $x_{0}$ be some point in $M$. Let $a_{n}^{\prime}=d\left(x_{n}, x_{0}\right)$ and $d_{n}^{\prime}=a_{n}^{\prime}-$ $d\left(x_{n},-\right)=d_{n}+a_{n}^{\prime}-a_{n}$. Since $\phi\left(x_{0}\right)=\lim d_{n}\left(x_{0}\right)=\lim \left(a_{n}-a_{n}^{\prime}\right)$, we have $\left\{d_{n}^{\prime}\right\}$ converges pointwise to $\phi-\phi\left(x_{0}\right)$, which represents the same element as $\phi$ in $\partial_{B}(M)$. Thus, we may assume that our functions $d_{n}$ are of the form of $d_{n}^{\prime}$, i.e., $d_{n}=d\left(x_{n}, x_{0}\right)-d\left(x_{n},-\right)$.

By restricting to an appropriate subsequence, we may assume that for $\gamma_{n}$ a minimizing unit-speed geodesic from some point $z$ to $x_{n}$, the geodesic segments $\left\{\gamma_{n}\right\}$ converge to a unit-speed geodesic $\gamma_{\infty}:[0, \omega) \rightarrow M$. Clearly, $\gamma_{\infty}$ is a ray, i.e., it is minimizing on its maximal domain, $[0, \omega)$; consequently, its Busemann function $b_{\gamma_{\infty}}$ is finite. We will see, in fact, that the hypothesis $\left(^{*}\right)$ yields (with choice of $\left.x_{0}=z\right) b_{\gamma_{\infty}}=\phi$. This will then establish both conclusion (1) (since $\phi$ is then in $\mathcal{I P}(M)$ ) and conclusion (2) (since $\gamma_{\infty}$ is a geodesic).

We know for all $x, \phi(x)=\lim d_{n}(x)=\lim \left(d\left(x_{n}, x_{0}\right)-d\left(x_{n}, x\right)\right)$. We have freedom to choose the arbitrary point $x_{0}$; letting $x_{0}=z$ then gives us $\phi(x)$ as the 
right side of $\left(^{*}\right)$. Since $\gamma_{\infty}$ is a ray commencing at $z$, for all $t<\omega, d\left(z, \gamma_{\infty}(t)\right)=t$; thus, $b_{\gamma_{\infty}}(x)$ is the left side of $(*)$ (which is why that limit always exists).

Note that the left side of $(* *)$ is $\lim _{n \rightarrow \infty}\left(t_{n}-d\left(x, \gamma_{\infty}\left(t_{n}\right)\right)\right)$, which is necessarily $b_{\gamma_{\infty}}(x)$. Thus, $\left({ }^{* *}\right)$ is also a sufficient condition.

Before looking at an application of this proposition, we should perhaps look at examples where it does not apply. If $M$ is the unwrapped grapefruit on a stick$\tilde{G}$ of section 2 -and $\left\{x_{n}\right\}$ is a sequence of points running out to infinity along the unwrapped equator, then, as $M$ is complete, for any $z$, there can be found a minimizing geodesic $\gamma_{n}$ from $z$ to $x_{n}$. If $z$ is in the upper flat region, for instance, then $\left\{\gamma_{n}\right\}$ converges to a geodesic $\gamma_{\infty}$ which is the horizontal geodesic starting at $z$. But $\left(^{*}\right)$ is not satisfied, as the left side is $b_{\gamma_{\infty}}\left(\right.$ or $b_{c^{+}}$), while the right side is $\infty$ (since the unwrapped equator is not asymptotically ray-like). If $z$ is in the round sector, then the convergence is a bit more interesting, as the geodesic segments $\gamma_{n}$ never enter the flat region; they stay in the round region and, for a long distance, in the tiny negative-curvature region between the flat and round regions. They still converge (or, in the case of $z$ lying right on the unwrapped equator, some subsequence of them converge) to a geodesic $\gamma_{\infty}$; but in this case $\gamma_{\infty}$ is a somewhat remarkable geodesic that is uniquely characterized as starting from $z$, entering the negative-curvature region (either the upper or lower one) and never leaving it. However, the Busemann function for this geodesic is exactly the same (up to a constant) as that for $c^{+}$(or $c^{-}$, for the lower negative-curvature region). Thus, $\left(^{*}\right)$ is never satisfied for $\left\{x_{n}\right\}$, and the Busemann boundary is non-Hausdorff: $\left\{d_{n}\right\}$ has a limit $\phi$ which is not in $\mathcal{I P}(M)$, but is the max of $\left\{b_{c^{+}}, b_{c^{-}}\right\}$(up to constants), and $\left[b_{c^{+}}\right]$and $\left[b_{c^{-}}\right]$are both chronological limits of $\left\{\left[d_{n}\right]\right\}$, i.e. of $\left\{x_{n}\right\}$.

If, instead of a single grapefruit, we unwrap an infinite string of them, all on a single stick, then we obtain a manifold $M$ which violates not conclusion (1) of Proposition 6.1, but conclusion (2). Let $x_{n}$ be on the unwrapped equator of the $n$th grapefruit, at a distance $\theta_{n}=n^{3}$ out from $\theta=0$. If we pick $z_{k}$ in the $k$ th flat region, then the geodesic segments $\left\{\gamma_{n}^{k}\right\}$ converge, as before, to a geodesic $\gamma_{\infty}^{k}=c_{k}$ which is a horizontal geodesic starting at $z_{k}$. The left-hand side of $\left(^{*}\right)$ is now $b_{c_{k}}$. The right-hand side, instead of being infinite, is equal to $\max \left\{b_{c_{n}}\right\}$; this is $b_{c}$ for $c$ the broken geodesic connecting the points $\left\{x_{n}\right\}$, a finite Busemann function but not the Busemann function of any geodesic.

In spite of the non-geodesic nature of the element $\left[b_{c}\right]$ of the Busemann boundary for $M, \partial_{B}(M)$ is, none the less, Hausdorff. This suggests a modification of Proposition 6.1: Suppose $M$ has distances realized by geodesics and every divergent sequence $\left\{x_{n}\right\}$ has a subsequence (call it again $\left\{x_{n}\right\}$ ) so that for some choice of minimizing unit-speed geodesic segments $\gamma_{n}^{m}$ from $x_{m}$ to $x_{n}$ (with $\gamma_{n}^{m}(0)=x_{m}$ ), for each fixed $m$, the geodesic segments $\left\{\gamma_{n}^{m} \mid n \geq m\right\}$ converge to a geodesic $\gamma_{\infty}^{m}$ with Busemann function $b_{\gamma_{\infty}^{m}}=b_{m}$ such that the sequence of functions $\left\{b_{m}-b_{m}\left(x_{0}\right)\right\}$ is monotonic increasing and converges pointwise to $\lim _{n \rightarrow \infty}\left(d\left(x_{0}, x_{n}\right)-d\left(-, x_{0}\right)\right)$; then the chronological topology on $\partial_{B}(M)$ is the same as the function-space topology. (Proof sketch: Let $\phi$ be the pointwise limit of $\left\{d_{n}\right\}$ for $d_{n}=d\left(x_{0}, x_{n}\right)-d\left(-, x_{0}\right)$. By Lemma 5.2, the pointwise limit of a monotonically increasing sequence of elements of $\mathcal{I P}(M)$ is in $\mathcal{I P}(M)$; apply this to $\phi$. Then, as in Proposition 6.1, it follows that $\partial_{B}(M)$ is Hausdorff.)

The motivation for Proposition 6.1 is its application to external Schwarzschild; 
this has as manifold $\mathbb{R} \times(2 m, \infty) \times \mathbb{S}^{2}$ and as metric $d s^{2}=-\left(1-\frac{2 m}{r}\right) d t^{2}+$ $\left(1-\frac{2 m}{r}\right)^{-1} d r^{2}+r^{2} d \mathbb{S}^{2}$, where $d \mathbb{S}^{2}$ denotes the round metric on the sphere. This is conformal to the metric $-d t^{2}+h=-d t^{2}+\left(1-\frac{2 m}{r}\right)^{-2} d r^{2}+r^{2}\left(1-\frac{2 m}{r}\right)^{-1} d \mathbb{S}^{2}$. Since the future causal boundary is conformally invariant, we can use this latter metric. Thus we have external Schwarzschild is conformal to the standard static spacetime $\mathbb{L}^{1} \times M$, where $M$ is topologically $(2 m, \infty) \times \mathbb{S}^{2}$. It will be much easier to treat $M$ as a warped product by defining $\rho(r)=\int\left(1-\frac{2 m}{r}\right)^{-1} d r=r+2 m \ln (r-2 m)$, yielding the metric $h$ on $M$ as $d \rho^{2}+r(\rho)^{2}\left(1-\frac{2 m}{r(\rho)}\right)^{-1} d \mathbb{S}^{2}$, a warped product metric on $(-\infty, \infty) \times \mathbb{S}^{2}$. Note that as $\rho$ goes to $\infty$, so does the warping function $r^{2}\left(1-\frac{2 m}{r}\right)^{-1}$; and as $\rho$ goes to $-\infty, r$ goes to $2 m$ and, again, the warping function goes to $\infty$. More specifically, the warping function is monotonic increasing on $\left[\rho_{0}, \infty\right)$ and monotonic decreasing on $\left(-\infty, \rho_{0}\right]$, where $r\left(\rho_{0}\right)=3 \mathrm{~m}$. This, in fact, is sufficient to allow us to conclude that the topology of the future causal boundary is a null cone on two copies of $\mathbb{S}^{2}$, as the next theorem establishes.

Theorem 6.2. Let $M$ be a Riemannian manifold expressible as a warped product with a compact manifold: $M=(\alpha, \omega) \times_{a} K$, where $(\alpha, \omega)$ is a possibly infinite interval in $\mathbb{R}, a:(\alpha, \omega) \rightarrow \mathbb{R}$ is a positive function, and $K$ is compact, and the metric on $M$ is given by $h=d \rho^{2}+a(\rho)^{2} j_{K}\left(j_{K}\right.$ being the metric on $\left.K\right)$.

Suppose that for some $\rho_{0} \in(\alpha, \omega), a(\rho)$ is monotonic decreasing for $\rho \in\left(\alpha, \rho_{0}\right]$ and monotonic increasing for $\rho \in\left[\rho_{0}, \omega\right)$. Then $M$ satisfies the hypotheses of Proposition 6.1, and $\partial_{B}(M)$ consists of spaces $B_{\alpha}$ and $B_{\omega}$, attached respectively at $\{\alpha\} \times K$ and $\{\omega\} \times K$, with each $B_{\iota}(\iota=\alpha, \omega)$ either $K$ or a single point $*$ : $B_{\iota} \cong K$ if $\left|\int_{\rho_{0}}^{\iota} 1 / a(\rho)^{2} d \rho\right|<\infty$, and $B_{\iota}=*$ if $\left|\int_{\rho_{0}}^{\iota} 1 / a(\rho)^{2} d \rho\right|=\infty$.

Thus, if $\int_{\alpha}^{\rho_{0}} 1 / a(\rho)^{2} d \rho$ and $\int_{\rho_{0}}^{\omega} 1 / a(\rho)^{2} d \rho$ are both finite, the future causal boundary (using the future chronological topology) for $V=\mathbb{L}^{1} \times(\alpha, \omega) \times{ }_{a} K$ consists of conjoined cones on two copies of $K$, one for each endpoint of the interval $(\alpha, \omega)$; the cone-elements corresponding to each endpoint are null if that endpoint is infinite, timelike if the endpoint is finite.

Proof. This is a quite lengthy proof, as there is much to be established. Part I is the proof that condition $(* *)$ of Proposition 6.1 is met; this requires a close consideration of the form that geodesics in $M$ take. Part II is the proof that the spaces $B_{\iota}$ have the form claimed: IIa for the infinite integral and IIb for the finite integral (the latter requiring the establishment of a homeomorphism with $K$ ).

\section{Part I: Applying Proposition 6.1.}

We need to establish what the distance function on $M$ is. Let $M^{-}=\left(\alpha, \rho_{0}\right) \times K$ and $M^{+}=\left(\rho_{0}, \omega\right) \times K$; we will look at how geodesics either stay in the one half or the other of $M$ or how they move from one half to the other.

Let $\gamma(s)=(\rho(s), c(s))$ be a geodesic in $M$; then the geodesic equation in $M$ gives us

$$
\ddot{\rho}=a a^{\prime}|\dot{c}|^{2} \quad \text { and } \quad \ddot{c}=-2 \dot{\rho} \frac{a^{\prime}}{a} \dot{c},
$$

where 'denotes $d / d s,^{\prime}$ denotes $d / d \rho$, and $\|$ denotes the norm in the metric on $K$. Thus, $c$ is a pregeodesic (reparametrization of an actual geodesic) in $K$, and we 
have the following integrations (taking $\gamma$ to be unit-speed):

$$
\dot{\rho}^{2}=1-\frac{a_{0}^{2} \sin ^{2} \theta_{0}}{a^{2}} \quad \text { and } \quad|\dot{c}|=\frac{a_{0} \sin \theta_{0}}{a^{2}}
$$

where $a_{0}=a(\rho(0))$ and $\theta_{0}$ is the angle between $\dot{\gamma}(0)$ and the radial vector field $\mathbf{R}$ in $M$ given by $(d / d \rho)(\rho, p)$ for fixed $p \in K$.

Note in particular that in the regime of $a$ being increasing, $\ddot{\rho}$ is positive, so if $\dot{\rho}$ starts off positive, it remains positive. Therefore, if $\rho(0) \geq \rho_{0}$ and $\theta_{0} \leq \pi / 2$ - and at least one of those inequalities is strict - then $\rho(s)$ will be a strictly increasing function of $s$, and we can reparametrize $\gamma$ by $\rho$, which greatly simplifies the analysis. If $\gamma$ goes from $x_{1}=\left(\rho_{1}, p_{1}\right)$ to $x_{2}=\left(\rho_{2}, p_{2}\right)$ and if we know $\rho(s)$ is strictly increasing, then let $\bar{\gamma}:\left[\rho_{1}, \rho_{2}\right] \rightarrow M$ be the parametrization of $\gamma$ by $\rho$, i.e, $\bar{\gamma}(\rho)=(\rho, \bar{c}(\rho))$, where $\bar{c}:\left[\rho_{1}, \rho_{2}\right] \rightarrow K$ is a reparametrization of $c$; then

$$
\begin{aligned}
L(\gamma) & =L(\bar{\gamma}) \\
& =\int_{\rho_{1}}^{\rho_{2}}\left|\frac{d \bar{\gamma}}{d \rho}\right| d \rho \\
& =\int_{\rho_{1}}^{\rho_{2}}\left|\frac{d s}{d \rho} \frac{d \gamma}{d s}\right| d \rho \\
& =\int_{\rho_{1}}^{\rho_{2}} \frac{1}{\left|\frac{d \rho}{d s}\right|} d \rho \\
& =\int_{\rho_{1}}^{\rho_{2}} \frac{d \rho}{\sqrt{1-\left(\frac{a_{1} \sin \theta_{0}}{a(\rho)}\right)^{2}}}
\end{aligned}
$$

where $a_{1}=a\left(\rho_{1}\right)$. For any numbers $\rho_{1}, \rho_{2}$, and $\theta_{0}$ with $\rho_{0} \leq \rho_{1} \leq \rho_{2}$, we can define

$$
J_{\rho_{1}}^{\rho_{2}}\left(\theta_{0}\right)=\int_{\rho_{1}}^{\rho_{2}} \frac{d \rho}{\sqrt{1-\left(\frac{a_{1} \sin \theta_{0}}{a(\rho)}\right)^{2}}} ;
$$

then if we know $\gamma$ is minimizing, we have $d\left(x_{1}, x_{2}\right)=J_{\rho_{1}}^{\rho_{2}}\left(\theta_{0}\right)$.

If, on the other hand, $\theta_{0}>\pi / 2$, then $\rho(s)$ is decreasing in $s$ until $\rho(s)$ reaches a value $\rho_{\text {min }}$ defined by $a\left(\rho_{\min }\right)=a_{1} \sin \theta_{0}$, at which point it becomes increasing (if there is no such $\rho_{\min }$-i.e., if $\sin \theta_{0}<a\left(\rho_{0}\right) / a_{1}$ for $\theta_{0}>\pi / 2$ - then either $\gamma$ is asymptotic to the $\rho=\rho_{0}$ curve or it leaves $M^{+}$and enters $M^{-}$, never returning to $M^{+}$). We can still parametrize $\gamma$ by $\rho$, so long as we are careful to do so in two segments, with domains $\left[\rho_{\min }, \rho_{1}\right]$ and $\left[\rho_{\min }, \rho_{2}\right]$. Then we obtain

$$
L(\gamma)=\int_{\rho_{\min }}^{\rho_{1}} \frac{d \rho}{\sqrt{1-\left(\frac{a_{1} \sin \theta_{0}}{a(\rho)}\right)^{2}}}+\int_{\rho_{\min }}^{\rho_{2}} \frac{d \rho}{\sqrt{1-\left(\frac{a_{1} \sin \theta_{0}}{a(\rho)}\right)^{2}}} .
$$

In this case, if $\gamma$ is minimizing, then $d\left(x_{1}, x_{2}\right)=J_{\rho_{\min }}^{\rho_{1}}\left(\theta_{0}\right)+J_{\rho_{\min }}^{\rho_{2}}\left(\theta_{0}\right)$.

In case $\rho_{1}<\rho_{0}<\rho_{2}$, we have $\ddot{\rho} \leq 0$ for $\rho \leq \rho_{0}$ and $\ddot{\rho} \geq 0$ for $\rho \geq \rho_{0}$. This means that $\rho$ is strictly increasing from $\rho_{1}$ to $\rho_{2}$ : If $\rho$ starts to decrease while $\rho<\rho_{0}$, it 
never can increase again, so it stays below $\rho_{0}$; but it must reach $\rho_{2}$. And since it is increasing when it passes $\rho_{0}$, it cannot decrease after that point. Thus, we can again use $\rho$ for the parametrization of $\gamma$; if $\gamma$ is minimizing, a similar expression obtains for $d\left(x_{1}, x_{2}\right)$.

Note that we have established that all geodesics bend towards $\rho=\rho_{0}$, in the following sense: Take the case of $\rho_{0} \leq \rho_{1} \leq \rho_{2}$; in going from $\left(\rho_{1}, p_{1}\right)$ to $\left(\rho_{2}, p_{2}\right)$, a geodesic either will have all its values of $\rho$ lying between $\rho_{1}$ and $\rho_{2}$, or, if it takes values of $\rho$ outside that range, those other values lie between $\rho_{0}$ and $\rho_{1}$. The case of $\rho_{1} \leq \rho_{2} \leq \rho_{0}$ is analogous: $\rho$-values for the geodesic all lie between $\rho_{1}$ and $\rho_{2}$ or, if they depart from that, take their additional values between $\rho_{2}$ and $\rho_{0}$. In the case of $\rho_{1} \leq \rho_{0} \leq \rho_{2}$, the $\rho$-values always fall between $\rho_{1}$ and $\rho_{2}$. It follows that distances in $M$ are realized by geodesics:

Consider any two points $x_{i}=\left(\rho_{i}, p_{i}\right), i=1,2$. Pick $\rho^{-}<\rho^{+}$so that $\rho_{0}, \rho_{1}$, and $\rho_{2}$ are all in $\left(\rho^{-}, \rho^{+}\right)$. Let $\bar{M}=(-\infty, \infty) \times_{\bar{a}} K$, where $\bar{a}$ agrees with $a$ on $\left(\rho^{-}, \rho^{+}\right)$, is constant off of $\left(\rho^{-}-\epsilon, \rho^{+}+\epsilon\right)$, obeys $\bar{a} \leq a$, and has the requisite monotonicity properties. Then $\bar{M}$ is obviously complete (differing only on a compact region from the product metric on $\left.\mathbb{R}^{1} \times K\right)$. Let $M_{0}=\left(\rho^{-}, \rho^{+}\right) \times{ }_{a} K$; this is an open submanifold of $M$ and also of $\bar{M}$. The preceding analysis on geodesics in $M$ applies to $\bar{M}$; thus, if $\bar{\gamma}$ is a minimizing $\bar{M}$-geodesic from $x_{1}$ to $x_{2}$, we know that $\bar{\gamma}$-bending towards $\rho=\rho_{0}$-remains within $M_{0}$, hence, within $M$. We can map $M$ into $\bar{M}$ by $i:(\rho, p) \mapsto(\rho, p)$; then $i$ is length-decreasing. It follows that $\bar{\gamma}$ is minimizing in $M$ as well as in $\bar{M}$, so it is also a minimizing $M$-geodesic. Thus, all distances in $M$ are realized by geodesics.

It follows that for any two points $x_{1}=\left(\rho_{1}, p_{1}\right)$ and $x_{2}=\left(\rho_{2}, p_{2}\right)$ with $\rho_{1} \leq \rho_{2}$, the distance between $x_{1}$ and $x_{2}$ can be expressed as

$$
d\left(x_{1}, x_{2}\right)=\oint_{\rho_{1}}^{\rho_{2}} \frac{d \rho}{\sqrt{1-\left(\frac{a\left(\rho_{1}\right) \sin \theta_{0}}{a(\rho)}\right)^{2}}}
$$

where $\theta_{0}$ is the angle made at $x_{1}$ between the radial vector field $\mathbf{R}$ and a minimizing geodesic from $x_{1}$ to $x_{2}$, and $\oint_{\rho_{1}}^{\rho_{2}}$ denotes either $\int_{\rho_{1}}^{\rho_{2}}$ or $\int_{\rho_{\min }}^{\rho_{1}}+\int_{\rho_{\min }}^{\rho_{2}}$ for the appropriate $\rho_{\min }$, depending on the value of $\theta_{0}$ (with appropriate readjustment of nomenclature in case $\left.\rho_{2}<\rho_{0}\right)$.

We must show that the hypotheses of Proposition 6.1 are satisfied by $M$. Let $\left\{x_{n}\right\}=\left\{\left(\rho_{n}, p_{n}\right)\right\}$ be a divergent sequence of points in $M$. As $K$ is compact, it must be that $\left\{\rho_{n}\right\}$ has no accumulation point in $(\alpha, \omega)$; we may assume that for some subsequence $\left\{\rho_{n}\right\}$ approaches $\omega$. Again using that $K$ is compact, we know that for some subsequence, $\left\{p_{n}\right\}$ converges to a limit $p_{\infty}$. We will, for now, let $z=\left(\rho_{z}, p_{\infty}\right)$ with $\rho_{z}$ unspecified. For each $n$, let $\gamma_{n}$ be a minimizing unit-speed geodesic from $z$ to $x_{n}$ (so $\left.\gamma_{n}(0)=z\right)$. For some subsequence, the vectors $\left\{\dot{\gamma}_{n}(0)\right\}$ converge to a unit-speed vector $v$ at $z$.

Lemma 6.3. The vector $v$ is outward-radial, i.e., $v=\mathbf{R}$.

Proof of Lemma. Let $z_{n}=\left(\rho_{z}, p_{n}\right)$, and let $\delta_{n}$ be the radial geodesic from $z_{n}$ to $x_{n}=\left(\rho_{n}, p_{n}\right)$; clearly, $L\left(\delta_{n}\right)=\rho_{n}-\rho_{z}$ (for $n$ so large that $\left.\rho_{n}>\rho_{z}\right)$. Let $\alpha_{n}$ be the $\rho=\rho_{z}$ curve from $z$ to $z_{n} ; L\left(\alpha_{n}\right)=a\left(\rho_{z}\right) d_{K}\left(p_{n}, p\right)$. Since $\gamma_{n}$ is minimizing, we have $L\left(\gamma_{n}\right) \leq L\left(\delta_{n}\right)+L\left(\alpha_{n}\right)$. We also have $L\left(\gamma_{n}\right)=\oint_{\rho_{z}}^{\rho_{n}} d \rho / \sqrt{1-\left(\left(a\left(\rho_{z}\right) \sin \theta_{n}\right) / a(\rho)\right)^{2}}$, where $\theta_{n}$ is the initial angle of $\gamma_{n}$ with $\mathbf{R}$. In the case that $\theta_{n}>\pi / 2$, the two 
integrals symbolized by $\oint_{\rho_{z}}^{\rho_{n}}$ contain, as a subinterval, the integral $\int_{\rho_{z}}^{\rho_{n}}$. Thus, in any case, we have

$$
\int_{\rho_{z}}^{\rho_{n}} \frac{d \rho}{\sqrt{1-\left(\frac{a\left(\rho_{z}\right) \sin \theta_{n}}{a(\rho)}\right)^{2}}}-\left(\rho_{n}-\rho_{z}\right) \leq a\left(\rho_{z}\right) d_{K}\left(p_{n}, p_{\infty}\right) .
$$

Combining those two terms on the left into a single integral, we have

$$
\int_{\rho_{z}}^{\rho_{n}}\left(\frac{1}{\sqrt{1-\left(\frac{a\left(\rho_{z}\right) \sin \theta_{n}}{a(\rho)}\right)^{2}}}-1\right) d \rho \leq a\left(\rho_{z}\right) d_{K}\left(p_{n}, p_{\infty}\right) .
$$

Using $\frac{1}{\sqrt{1-x}} \geq 1+(1 / 2) x$, we get

$$
\sin ^{2} \theta_{n} \int_{\rho_{z}}^{\rho_{n}} \frac{d \rho}{a(\rho)^{2}} \leq \frac{2}{a\left(\rho_{z}\right)} d_{K}\left(p_{n}, p_{\infty}\right)
$$

Since $\left\{p_{n}\right\}$ approaches $p_{\infty}$, those distances $d_{K}\left(p_{n}, p_{\infty}\right)$ approach 0 . On the other side of the inequality, the integrals are increasing in $n$. It follows that $\left\{\sin \theta_{n}\right\}$ must approach 0. It's not possible that any subsequence of $\left\{\theta_{n}\right\}$ approach $\pi$ (since that would have $\gamma_{n}$ entering $M^{-}$and not making it to $x_{n}$, for $n$ sufficiently large), so $\left\{\theta_{n}\right\}$ must approach 0, i.e., $\left\{\dot{\gamma}_{n}(0)\right\}$ approaches $\mathbf{R}$.

We will choose $\rho_{z}=\rho_{0}$, the value for $\rho$ which minimizes $a(\rho)$. We have the geodesic segments $\left\{\gamma_{n}\right\}$ converging to the geodesic $\gamma_{\infty}$ defined by $\dot{\gamma}_{\infty}(0)=\mathbf{R}$, $\gamma_{\infty}:\left[0, \omega-\rho_{0}\right) \rightarrow M$. We will show condition (**) of Proposition 6.1 holds if we select $t_{n}=\rho_{n}-\rho_{0}$ for each $n$ (so $\gamma_{\infty}\left(t_{n}\right)=\left(\rho_{n}, p_{\infty}\right)$ ); that is to say, we must show, for all $x \in M$,

$$
\lim _{n \rightarrow \infty}\left(d\left(z, \gamma_{\infty}\left(t_{n}\right)\right)-d\left(x, \gamma_{\infty}\left(t_{n}\right)\right)\right)=\lim _{n \rightarrow \infty}\left(d\left(z, x_{n}\right)-d\left(x, x_{n}\right)\right)
$$

We will do this by showing

$$
\lim _{n \rightarrow \infty}\left(d\left(z, \gamma_{\infty}\left(t_{n}\right)\right)-d\left(z, x_{n}\right)\right)=0
$$

and

$$
\lim _{n \rightarrow \infty}\left(d\left(x, \gamma_{\infty}\left(t_{n}\right)\right)-d\left(x, x_{n}\right)\right)=0
$$

(Showing $(\dagger)$ and $(\ddagger)$ will suffice, whether the left side of $(* *)$ is finite or infinite.) As can be seen, $(\dagger)$ is just a specialization of $(\ddagger)$ to the case of $x=z$; thus $(\ddagger)$ is all we need be concerned with.

We will need the following lemma relating the distance function $d$ in $M$ to the distance function $d_{K}$ in $K$. 
Lemma 6.4. Given four points in $K, p_{1}, p_{2}, p_{1}^{\prime}$, and $p_{2}^{\prime}$ satisfying $d_{K}\left(p_{1}, p_{2}\right) \leq$ $d_{K}\left(p_{1}^{\prime}, p_{2}^{\prime}\right)$, and any two numbers $\rho_{1}$ and $\rho_{2}$ in $(\alpha, \omega)$,

$$
d\left(\left(\rho_{1}, p_{1}\right),\left(\rho_{2}, p_{2}\right)\right) \leq d\left(\left(\rho_{1}, p_{1}^{\prime}\right),\left(\rho_{2}, p_{2}^{\prime}\right)\right) .
$$

Proof of Lemma. Let $z_{i}=\left(\rho_{i}, p_{i}\right)$ and $z_{i}^{\prime}=\left(\rho_{i}, p_{i}^{\prime}\right)$ for $i=1,2$. We must show $d\left(z_{1}, z_{2}\right) \leq d\left(z_{1}^{\prime}, z_{2}^{\prime}\right)$. Let $\gamma^{\prime}$ be a minimizing geodesic from $z_{1}^{\prime}$ to $z_{2}^{\prime}$. We know we can parametrize $\gamma^{\prime}$ by $\rho$, save that we may have to split it into two segments. Let us first assume that we do not need two segments; then, taking $\rho_{1} \leq \rho_{2}$, we know that $\gamma^{\prime}$ is expressible as $\gamma^{\prime}(\rho)=\left(\rho, \sigma^{\prime}(\alpha(\rho))\right)$, where $\sigma^{\prime}$ is a geodesic in $K$ from $p_{1}^{\prime}$ to $p_{2}^{\prime}$ and $\alpha$ is some reparametrization; if we take $\sigma^{\prime}$ to be unit-speed, then $\alpha\left(\rho_{1}\right)=0$ and $\alpha\left(\rho_{2}\right)=L\left(\sigma^{\prime}\right)$. (Note that it is entirely possible that $\sigma^{\prime}$ is not a minimizing geodesic, even though $\gamma^{\prime}$ is.) We have $d\left(z_{1}^{\prime}, z_{2}^{\prime}\right)=L\left(\gamma^{\prime}\right)=\int_{\rho_{1}}^{\rho_{2}} \sqrt{1+a(\rho)^{2} \dot{\alpha}(\rho)^{2}} d \rho$ (with $\dot{\alpha}=d \alpha / d \rho$ ).

Let $\sigma$ be a minimizing unit-speed geodesic in $K$ from $p_{1}$ to $p_{2}$. We know $L(\sigma)=d_{K}\left(p_{1}, p_{2}\right) \leq d_{K}\left(p_{1}^{\prime}, p_{2}^{\prime}\right) \leq L\left(\sigma^{\prime}\right)$; let $f=L(\sigma) / L\left(\sigma^{\prime}\right)$. Define the curve $\gamma:\left[\rho_{1}, \rho_{2}\right] \rightarrow M$ by $\gamma(\rho)=(\rho, \sigma(f \alpha(\rho)))$; then $\gamma$ goes from $z_{1}$ to $z_{2}$. We have $d\left(z_{1}, z_{2}\right) \leq L(\gamma)=\int_{\rho_{1}}^{\rho_{2}} \sqrt{1+a(\rho)^{2} f^{2} \dot{\alpha}(\rho)^{2}} d \rho \leq L\left(\gamma^{\prime}\right)=d\left(z_{1}^{\prime}, z_{2}^{\prime}\right)$, as desired.

Now suppose that $\gamma^{\prime}$ is expressed as a portion $\beta^{\prime}$ parametrized by $\rho$ on $\left[\rho_{\min }^{\prime}, \rho_{1}^{\prime}\right]$ and a portion $\delta^{\prime}$ parametrized by $\rho$ on $\left[\rho_{\text {min }}^{\prime}, \rho_{2}^{\prime}\right]$. We still have a geodesic $\sigma^{\prime}$ in $K$ from $p_{1}^{\prime}$ to $p_{2}^{\prime}$ providing (after reparametrization) the second coordinate for each of the segments $\beta^{\prime}$ and $\delta^{\prime}$; the only difference from before is that the reparametrization in $\beta^{\prime}$ runs backwards. The same technique as above yields a curve $\gamma$ from $z_{1}$ to $z_{2}$, using the same parametrizations by $\rho$ as $\gamma^{\prime}$ uses, again yielding $L(\gamma) \leq L\left(\gamma^{\prime}\right)$, whence $d\left(z_{1}, z_{2}\right) \leq d\left(z_{1}^{\prime}, z_{2}^{\prime}\right)$.

We need to show $\lim _{n \rightarrow \infty}\left(d\left(x, \gamma_{\infty}\left(t_{n}\right)\right)-d\left(x, x_{n}\right)\right)=0$. Represent $x$ as $x=$ $\left(\rho_{x}, p_{x}\right)$.

Suppose $\lim \sup \left(d\left(x, \gamma_{\infty}\left(t_{n}\right)\right)-d\left(x, x_{n}\right)\right)>0$, i.e., for some subsequence, for some $\epsilon>0$, for all $k, d\left(x, \gamma_{\infty}\left(t_{n_{k}}\right)\right) \geq d\left(x, x_{n_{k}}\right)+\epsilon$. We will define a sequence of points $\left\{q_{k}\right\}$ approaching $p_{x}$ with distances from $p_{\infty}$ mirroring the distances of $p_{n_{k}}$ from $p_{x}$; the idea is to be able to apply Lemma 6.4 to each quartet of points $\left\{\left(q_{k}, p_{\infty}\right),\left(p_{n_{k}}, p_{x}\right)\right\}$. If $p_{x}=p_{\infty}$, then we just let each $q_{k}=p_{\infty}$ (thus clearly yielding $\left.d_{K}\left(q_{k}, p_{\infty}\right) \leq d_{K}\left(p_{n_{k}}, p_{x}\right)\right)$. If $p_{x} \neq p_{\infty}$, then we will choose the points $\left\{q_{k}\right\}$ along a path from $p_{\infty}$ to $p_{x}$. Specifically, let $\sigma$ be a minimizing geodesic in $K$ from $p_{\infty}$ to $p_{x}$ with $\sigma(0)=p_{\infty}$ and $\sigma(1)=p_{x}$. Define $q_{k}=\sigma\left(s_{k}\right)$ where $s_{k}=\min \left\{1, d_{K}\left(p_{x}, p_{n_{k}}\right) / d_{K}\left(p_{x}, p_{\infty}\right)\right\}$; note $\left\{s_{k}\right\}$ converges to 1 . Then we have $d_{K}\left(q_{k}, p_{\infty}\right)=s_{k} d_{K}\left(p_{x}, p_{\infty}\right) \leq d_{K}\left(p_{x}, p_{n_{k}}\right)$ for all $k$, while $\left\{q_{k}\right\}$ converges to $p_{x}$.

For all $k$, let $y_{k}=\left(\rho_{x}, q_{k}\right)$. Let $c_{k}$ be the curve from $x$ to $y_{k}$ of constant $\rho$ given by $c_{k}(s)=\left(\rho_{x}, \sigma(1-s)\right.$ ) (defined for $\left.s \leq 1-s_{k}\right)$, and let $\delta_{k}$ be a minimizing geodesic in $M$ from $y_{k}$ to $\gamma_{\infty}\left(t_{n_{k}}\right)$; finally, let $\beta_{k}$ be the concatenation of $c_{k}$ with $\delta_{k}$, a curve from $x$ to $\gamma_{\infty}\left(t_{n_{k}}\right)$. On the one hand, we have $L\left(\beta_{k}\right)=L\left(c_{k}\right)+d\left(y_{k}, \gamma_{\infty}\left(t_{n_{k}}\right)\right) \geq d\left(x, \gamma_{\infty}\left(t_{n_{k}}\right)\right) \geq d\left(x, x_{n_{k}}\right)+\epsilon$ (that last inequality by assumption), so $d\left(y_{k}, \gamma_{\infty}\left(t_{n_{k}}\right)\right) \geq d\left(x, x_{n_{k}}\right)+\epsilon-L\left(c_{k}\right)$; thus, for $k$ sufficiently large, $d\left(y_{k}, \gamma_{\infty}\left(t_{n_{k}}\right)\right) \geq d\left(x, x_{n_{k}}\right)+\epsilon / 2$ (as $\left\{L\left(c_{k}\right)\right\}$ converges to 0$)$. On the other hand, we can apply Lemma 6.4 to $y_{k}=\left(\rho_{x}, q_{k}\right), \gamma_{\infty}\left(t_{n_{k}}\right)=\left(\rho_{n_{k}}, p_{\infty}\right)$, $x=\left(\rho_{x}, p_{x}\right)$, and $x_{n_{k}}=\left(\rho_{n_{k}}, p_{n_{k}}\right)$, since $d_{K}\left(q_{k}, p_{\infty}\right) \leq d_{K}\left(p_{x}, p_{n_{k}}\right)$; that yields $d\left(y_{k}, \gamma_{\infty}\left(t_{n_{k}}\right)\right) \leq d\left(x, x_{n_{k}}\right)$ for all $k$. Thus the supposition leads to a contradiction. 
Now suppose $\lim \inf \left(d\left(x, \gamma_{\infty}\left(t_{n}\right)\right)-d\left(x, x_{n}\right)\right)<0$, i.e., for some subsequence, for some $\epsilon>0$, for all $k, d\left(x, \gamma_{\infty}\left(t_{n_{k}}\right)\right) \leq d\left(x, x_{n_{k}}\right)-\epsilon$. Let $\Delta=d_{K}\left(p_{x}, p_{\infty}\right)$. This time choose the sequence $\left\{q_{k}\right\}$ convergent to $p_{x}$ so that for all $k, q_{k}$ is no more than $K$-distance $\Delta$ from $p_{n_{k}}$; since $\left\{p_{n_{k}}\right\}$ converges to $p_{\infty}$, this can be accomplished (if $p_{n_{k}}$ is $\delta_{k}$ distant from $p_{\infty}$, it is no more than $\Delta+\delta_{k}$ distant from $p_{x}$, so the $\Delta$-ball around $p_{n_{k}}$ contains points within $2 \delta_{k}$ of $p_{x}$; let $q_{k}$ be one such). As before, let $y_{k}=\left(\rho_{x}, q_{k}\right)$ and $c_{k}$ be the $\rho$-constant curve from $x$ to $y_{k}$. But this time let $\delta_{k}$ be a minimizing geodesic in $M$ from $y_{k}$ to $x_{n_{k}}$, with $\beta_{k}$ the concatenation of $c_{k}$ with $\delta_{k}$, running from $x$ to $x_{n_{k}}$. Then we have, by assumption, $d\left(x, \gamma_{\infty}\left(t_{n_{k}}\right)\right)+\epsilon \leq$ $d\left(x, x_{n_{k}}\right) \leq L\left(\beta_{k}\right)=d\left(y_{k}, x_{n_{k}}\right)+L\left(c_{k}\right)$, so $d\left(y_{k}, x_{n_{k}}\right) \geq d\left(x, \gamma_{\infty}\left(t_{n_{k}}\right)\right)+\epsilon-L\left(c_{k}\right)$; thus, for $k$ sufficiently large, $d\left(y_{k}, x_{n_{k}}\right) \geq d\left(x, \gamma_{\infty}\left(t_{n_{k}}\right)\right)+\epsilon / 2$. Again we employ Lemma 6.4: We have the same coordinates in $\mathbb{R}$ and $K$ as before, but this time $d_{K}\left(q_{k}, p_{n_{k}}\right) \leq d_{K}\left(p_{x}, p_{\infty}\right)$, so we conclude $d\left(y_{k}, x_{n_{k}}\right) \leq d\left(x, \gamma_{\infty}\left(t_{n_{k}}\right)\right)$. Thus this supposition also leads to contradiction. (Actually, this part can be proved more generally, i.e., without reference to Lemma 6.4; but that achieves no simplicity of proof.)

The only remaining possibility is $\lim \left(d\left(x, \gamma_{\infty}\left(t_{n}\right)\right)-d\left(x, x_{n}\right)\right)=0$. This establishes the hypotheses in Proposition 6.1. Thus, $\partial_{B}(M)$ is the same in the chronological topology as in the function-space topology, and all its elements come from geodesics - indeed, geodesics of the form $\gamma_{\infty}$, i.e., radial geodesics of the form $\gamma_{p}^{+}:\left[0, \omega-\rho_{0}\right) \rightarrow M$ for $p \in K, \gamma_{p}^{+}(s)=\left(\rho_{0}+s, p\right)$, and $\gamma_{p}^{-}:\left[0, \rho_{0}-\alpha\right) \rightarrow M$, $\gamma_{p}^{-}(s)=\left(\rho_{0}-s, p\right)$. Thus, $\partial_{B}(M)$ consists of $B_{\omega}=\left\{\left[b_{\gamma_{p}^{+}}\right] \mid p \in K\right\}$ and $B_{\alpha}=$ $\left\{\left[b_{\gamma_{p}^{-}}\right] \mid p \in K\right\}$. We need to see how integral conditions on a determine these spaces to be either $K$ or a single point.

\section{Part II: Examining the boundary.}

We will confine discussion to $B_{\omega}$, as the arguments are identical for $B_{\alpha}$. We will let $b_{p}=b_{\gamma_{p}^{+}}$. Note that each $b_{p}$ is finite, as $\gamma_{p}^{+}$is a ray.

We will need to make use of integral functions involved in the distance formulas in $M$ and in $K$.

Recall the function

$$
J_{\rho_{1}}^{\rho_{2}}(\theta)=\int_{\rho_{1}}^{\rho_{2}} \frac{d \rho}{\sqrt{1-\left(\frac{a\left(\rho_{1}\right) \sin \theta}{a(\rho)}\right)^{2}}},
$$

defined for $\rho_{0} \leq \rho_{1} \leq \rho_{2}$; for a geodesic $\gamma$ in $M$ from $\left(\rho_{1}, p_{1}\right)$ to $\left(\rho_{2}, p_{2}\right)$ with initial radial angle of $\theta$, if $\theta \leq \pi / 2$, then $L(\gamma)=J_{\rho_{1}}^{\rho_{2}}(\theta)$. Note that $J_{\rho_{1}}^{\rho_{2}}$ is strictly increasing on $[0, \pi / 2]$.

We know that the projection to $K$ of any $M$-geodesic is a reparametrized $K$ geodesic. Thus, we can characterize all unit-speed geodesics in $M$ starting from a point $x_{1}=\left(\rho_{1}, p_{1}\right)$ as being of the form $\gamma_{\theta}^{\sigma}$, where $\sigma$ is any unit-speed geodesic in $K$ starting from $p_{1}$ and $\theta$ is any angle in $[0, \pi]$, and $\gamma_{\theta}^{\sigma}$ is defined by $\gamma_{\theta}^{\sigma}(s)=$ $\left(r_{\theta}(s), c_{\theta}^{\sigma}(s)\right)$ with $c_{\theta}^{\sigma}=\sigma \circ t_{\theta}$, where $r_{\theta}$ and $t_{\theta}$ are functions characterized by

$$
\begin{aligned}
\left(\dot{r}_{\theta}\right)^{2} & =1-\left(\frac{a\left(\rho_{1}\right) \sin \theta}{a\left(r_{\theta}\right)}\right)^{2}, & r_{\theta}(0) & =\rho_{1} \\
\dot{t}_{\theta} & =\frac{a\left(\rho_{1}\right) \sin \theta}{a\left(r_{\theta}\right)^{2}}, & t_{\theta}(0) & =0 .
\end{aligned}
$$


(Note that $\left|\dot{c}_{\theta}^{\sigma}\right|=\dot{t}_{\theta}$, so we are just using the same differential equations as before.) Assuming $\theta \leq \pi / 2$, let $\bar{c}$ be the reparametrization of $c_{\theta}^{\sigma}$ by $\rho$. Then we have

$$
\begin{aligned}
L(\sigma) & =L\left(c_{\theta}^{\sigma}\right)=L(\bar{c}) \\
& =\int_{\rho_{1}}^{\rho_{2}}\left|\frac{d \bar{c}}{d \rho}\right| d \rho \\
& =\int_{\rho_{1}}^{\rho_{2}}\left|\frac{d s}{d \rho} \frac{d c_{\theta}^{\sigma}}{d s}\right| d \rho \\
& =\int_{\rho_{1}}^{\rho_{2}} \frac{\left|\dot{c}_{\theta}^{\sigma}\right|}{\left|\frac{d \rho}{d s}\right|} d \rho \\
& =\int_{\rho_{1}}^{\rho_{2}} \frac{\dot{t}_{\theta}}{\dot{r}_{\theta}} d \rho \\
& =\int_{\rho_{1}}^{\rho_{2}} \frac{1}{\sqrt{1-\left(\frac{a\left(\rho_{1}\right) \sin \theta}{a(\rho)}\right)^{2}} \frac{a\left(\rho_{1}\right) \sin \theta}{a(\rho)^{2}} d \rho} \\
& =\int_{\rho_{1}}^{\rho_{2}} \frac{d \rho}{a(\rho) \sqrt{\left(\frac{a(\rho)}{a\left(\rho_{1}\right) \sin \theta}\right)^{2}-1}}=I_{\rho_{1}}^{\rho_{2}}(\theta) .
\end{aligned}
$$

Note $I_{\rho_{1}}^{\rho_{2}}$, like $J_{\rho_{1}}^{\rho_{2}}$, is strictly increasing on $[0, \pi / 2]$.

It is also convenient to express $I_{\rho_{1}}^{\rho_{2}}$ thus:

$$
I_{\rho_{1}}^{\rho_{2}}(\theta)=a\left(\rho_{1}\right) \sin \theta \int_{\rho_{1}}^{\rho_{2}} \frac{d \rho}{a(\rho)^{2} \sqrt{1-\left(\frac{a\left(\rho_{1}\right) \sin \theta}{a(\rho)}\right)^{2}}} .
$$

For an arbitrary geodesic $\gamma$ in $M$, its projection to $K$ is a reparametrization of a geodesic $\sigma_{\gamma}$ in $K$. In general, if $\gamma$ is minimizing, it does not necessarily follow that $\sigma_{\gamma}$ is minimizing. But it does if the initial radial angle $\theta_{\gamma}$ is no more than $\pi / 2$.

Lemma 6.5. Let $\gamma$ be a geodesic in $M$ from $x_{1}=\left(\rho_{1}, p_{1}\right)$ to $x_{2}=\left(\rho_{2}, p_{2}\right)$ with $\rho_{0} \leq \rho_{1} \leq \rho_{2}$. Assume the initial radial angle $\theta_{\gamma} \leq \pi / 2$. If $\gamma$ is minimizing, then so is the projected geodesic $\sigma_{\gamma}$ in $K$.

Proof of lemma. Suppose $\sigma_{\gamma}$ is not minimizing; then there is a geodesic $\bar{\sigma}$ from $p_{1}$ to $p_{2}$ which is minimizing. We have $L(\bar{\sigma})<L\left(\sigma_{\gamma}\right)=I_{\rho_{1}}^{\rho_{2}}\left(\theta_{\gamma}\right)$. Then there is a $\bar{\theta}<\theta_{\gamma}$ with $I_{\rho_{1}}^{\rho_{2}}(\bar{\theta})=L(\bar{\sigma})$. Note that $\gamma_{\bar{\theta}}^{\bar{\sigma}}$ is a geodesic from $x_{1}$ to $x_{2}$. We have $L\left(\gamma_{\bar{\theta}}^{\bar{\sigma}}\right)=J_{\rho_{1}}^{\rho_{2}}(\bar{\theta})<J_{\rho_{1}}^{\rho_{2}}\left(\theta_{\gamma}\right)=L(\gamma)$, implying $\gamma$ is not minimizing.

It follows that if there is a minimizing geodesic $\gamma$ from $\left(\rho_{1}, p_{1}\right)$ to $\left(\rho_{2}, p_{2}\right)$ which has initial radial angle $\theta_{\gamma} \leq \pi / 2$, then the distance between $p_{1}$ and $p_{2}$ is given by $d_{K}\left(p_{1}, p_{2}\right)=I_{\rho_{1}}^{\rho_{2}}\left(\theta_{\gamma}\right)$, since Lemma 6.5 yields that the projection $\sigma_{\gamma}$ is minimizing.

Note. While $\sigma_{\gamma}$, for minimizing $\gamma$ but $\theta_{\gamma}>\pi / 2$, need not be minimizing, it never the less follows from Lemma 6.5 that $\sigma_{\gamma}$ is the union of at most two minimizing geodesics: For consider that $\gamma$ is the union of two segments, $\gamma_{1}$ and $\gamma_{2}$, with $\gamma_{1}$ going from $\left(\rho_{1}, p_{1}\right)$ to some $\left(\rho_{\min }, \bar{p}\right)$ and $\gamma_{2}$ from $\left(\rho_{\min }, \bar{p}\right)$ to $\left(\rho_{2}, p_{2}\right)$. Note that $\theta_{\gamma_{2}}=$ $\pi / 2$, so $\sigma_{\gamma_{2}}$ is minimizing. Now consider that $\gamma_{1}$ can be considered in its reverse 
parametrization, $-\gamma_{1}$, running from $\left(\rho_{\min }, \bar{p}\right)$ to $\left(\rho_{1}, p_{1}\right)$; this produces $\theta_{-\gamma_{1}}=$ $\pi / 2$, so $\sigma_{-\gamma_{1}}$ is minimizing. Thus, $\sigma_{\gamma}$ is the concatenation of the two minimizing geodesics $-\sigma_{-\gamma_{1}}$ and $\sigma_{\gamma_{2}}$. (This perspective will be useful in the consideration of Reissner-Nordström.)

IIa: Infinite integral.

Suppose first that $\int_{\rho_{0}}^{\omega} 1 / a(\rho)^{2} d \rho=\infty$; we need to see that all $\left[b_{p}\right]$ are the same.

First note that we must have $\omega=\infty$ : Since $a$ is increasing on $\left[\rho_{0}, \omega\right), 1 / a(\rho)^{2}$ is decreasing for $\rho$ approaching $\omega$, so only integration over an infinite interval can yield an infinite integral.

Let us evaluate $b_{p}$ on an arbitrary point $(\bar{\rho}, q)$ :

$$
\begin{aligned}
b_{p}((\bar{\rho}, q)) & =\lim _{s \rightarrow \infty}\left(s-d\left(\left(\rho_{0}+s, p\right),(\bar{\rho}, q)\right)\right) \\
& =\lim _{s \rightarrow \infty}\left(-\rho_{0}+s-d((s, p),(\bar{\rho}, q))\right) \\
& =\lim _{s \rightarrow \infty}\left(-\rho_{0}+s-\oint_{\bar{\rho}}^{s} \frac{d \rho}{\sqrt{1-\left(\frac{\bar{a} \sin \theta_{s}}{a(\rho)}\right)^{2}}}\right),
\end{aligned}
$$

where $\bar{a}=a(\bar{\rho}), \theta_{s}$ is the initial radial angle for a minimizing geodesic from $(\bar{\rho}, q)$ to $(s, p)$, and $\oint$ represents either one or two integrals depending on whether $\theta_{s}$ is, respectively, $\leq \pi / 2$ or $>\pi / 2$.

Let us first assume that eventually $\theta_{s} \leq \pi / 2$. Then

$$
\begin{aligned}
b_{p}((\bar{\rho}, q)) & =-\rho_{0}+\lim _{s \rightarrow \infty}\left(s-\int_{\bar{\rho}}^{s} \frac{d \rho}{\sqrt{1-\left(\frac{\bar{a} \sin \theta_{s}}{a(\rho)}\right)^{2}}}\right) \\
& =-\rho_{0}+\bar{\rho}+\lim _{s \rightarrow \infty} \int_{\bar{\rho}}^{s}\left(1-\frac{1}{\sqrt{1-\left(\frac{\bar{a} \sin \theta_{s}}{a(\rho)}\right)^{2}}}\right) d \rho .
\end{aligned}
$$

Let $A(\theta)=\sqrt{1-\left(\frac{\bar{a} \sin \theta}{a(\rho)}\right)^{2}} ;$ then, using $1-\frac{1}{\sqrt{1-x}}=\frac{-x}{\sqrt{1-x}(\sqrt{1-x}+1)}$, we get

$$
b_{p}((\bar{\rho}, q))=-\rho_{0}+\bar{\rho}-\lim _{s \rightarrow \infty}\left(\bar{a}^{2} \sin ^{2} \theta_{s} \int_{\bar{\rho}}^{s} \frac{d \rho}{a(\rho)^{2} A\left(\theta_{s}\right)\left(A\left(\theta_{s}\right)+1\right)}\right) .
$$

Let $J_{s}$ denote the integral above. We have $A\left(\theta_{s}\right) \leq 1$, so $J_{s} \geq(1 / 2) \int_{\bar{\rho}}^{s} \frac{d \rho}{a(\rho)^{2}}$; therefore, our integral assumption on $a$ implies that $\lim _{s \rightarrow \infty} J_{s}=\infty$. Since we know that $b_{p}$ cannot have $-\infty$ as a value, we conclude that $\sin \theta_{s}$ must approach 0 . Since $\theta_{s} \leq \pi / 2$, this means $\lim _{s \rightarrow \infty} \theta_{s}=0$.

From Lemma 6.5, we know that for all $s, d_{K}(q, p)=I_{\bar{\rho}}^{s}\left(\theta_{s}\right)$, i.e.,

$$
d_{K}(q, p)=\bar{a} \sin \theta_{s} \int_{\bar{\rho}}^{s} \frac{d \rho}{a(\rho)^{2} A\left(\theta_{s}\right)} .
$$


Denote this integral by $I_{s}$; we have $\lim _{s \rightarrow \infty} I_{s}=\infty$, also. Solving this equation for $\bar{a} \sin \theta_{s}$ and inserting in the equation above it yields

$$
\begin{aligned}
b_{p}((\bar{\rho}, q)) & =-\rho_{0}+\bar{\rho}-\lim _{s \rightarrow \infty}\left(\frac{d_{K}(q, p)}{I_{s}}\right)^{2} J_{s} \\
& =-\rho_{0}+\bar{\rho}-d_{K}(q, p)^{2} \lim _{s \rightarrow \infty}\left(\frac{J_{s}}{I_{s}}\right) \frac{1}{I_{s}} .
\end{aligned}
$$

The only difference between $J_{s}$ and $I_{s}$ is that $J_{s}$ has an additional factor of $1 /\left(A\left(\theta_{s}\right)+1\right)$ in the integrand; therefore, $J_{s} \leq I_{s}$. Since $I_{s}$ has $\infty$ as limit, we conclude

$$
b_{p}((\bar{\rho}, q))=-\rho_{0}+\bar{\rho}
$$

under the assumption that eventually $\theta_{s} \leq \pi / 2$.

Now we look at the possibility that for some sequence of numbers $\left\{s_{n}\right\}$ approaching $\infty, \theta_{s_{n}}>\pi / 2$ for all $n$. We proceed much as before, utilizing the fact that for $\theta>\pi / 2, \oint_{\alpha}^{\beta}=\int_{\alpha}^{\beta}+2 \int_{\rho_{\min }}^{\alpha}$, where $a\left(\rho_{\min }\right)=\bar{a} \sin \theta$. Using $\theta_{n}$ for $\theta_{s_{n}}$ and $\rho_{n}$ for $a^{-1}\left(\bar{a} \sin \theta_{n}\right)$, we get

$$
\begin{aligned}
& b_{p}((\bar{\rho}, q))= \lim _{n \rightarrow \infty}\left(-\rho_{0}+s_{n}-\oint_{\bar{\rho}}^{s_{n}} \frac{d \rho}{A\left(\theta_{n}\right)}\right) \\
&= \lim _{n \rightarrow \infty}\left(-\rho_{0}+s_{n}-\int_{\bar{\rho}}^{s_{n}} \frac{d \rho}{A\left(\theta_{n}\right)}-2 \int_{\rho_{n}}^{\bar{\rho}} \frac{d \rho}{A\left(\theta_{n}\right)}\right) \\
&=-\rho_{0}+\bar{\rho}-\lim _{n \rightarrow \infty}\left(\bar{a}^{2} \sin ^{2} \theta_{n} \int_{\bar{\rho}}^{s_{n}} \frac{d \rho}{a(\rho)^{2} A\left(\theta_{n}\right)\left(A\left(\theta_{n}\right)+1\right)}\right) \\
& \quad-\lim _{n \rightarrow \infty} 2 \int_{\rho_{n}}^{\bar{\rho}} \frac{d \rho}{A\left(\theta_{n}\right)} .
\end{aligned}
$$

The only difference from before is the last term. That has no effect on the observation that since $b_{p}$ cannot take $-\infty$ as a value, we must have $\sin \theta_{n}$ approach 0 (which, under the current assumption, means $\theta_{n}$ approaches $\pi$ ). But that means $a\left(\rho_{n}\right)=\bar{a} \sin \theta_{n}$ approaches 0 , which is impossible, as $a(\rho) \geq a\left(\rho_{0}\right)$ for all $\rho$. We conclude that we cannot have the sequence $\left\{s_{n}\right\}$ after all.

It follows that $b_{p}((\bar{\rho}, q))=-\rho_{0}+\bar{\rho}$ for all $p, \bar{\rho}$, and $q$, i.e., $b_{p}$, up to a constant, is just projection onto the first coordinate -independent of $p$. Therefore, all $\left[b_{p}\right]$ are equal: $B_{\omega}$ is a point.

\section{IIb: Finite integral.}

Now suppose $\int_{\rho_{0}}^{\omega} 1 / a(\rho)^{2} d \rho<\infty$; we need to see that $B_{\omega}=\left\{\left[b_{p}\right] \mid p \in K\right\}$ is homeomorphic to $K$. First note that each $c_{p}: s \mapsto\left(\rho_{0}+s, p\right)$ is a ray, so $b_{p}$ is a finite Busemann function; thus, we have the map $i: K \rightarrow B_{\omega}$ given by $i: p \mapsto\left[b_{p}\right]$, and this is onto in virtue of the applicability of Proposition 6.1. Our task is to show $i$ is continuous and injective and that $i^{-1}$ is continuous.

\section{Showing $i$ is continuous.}

We can express $i$ as $i=\pi \circ j$, where $j: K \rightarrow \mathcal{B}(M)$ is the map into the space of Busemann functions, $j: p \mapsto b_{p}$, and $\pi$ is the quotient by the $\mathbb{R}$-action. We need to show that $j$ is continuous; as $V_{0}^{*}$ carries the quotient topology from the space of 
Busemann functions, $\pi$ is necessarily continuous, so that will suffice. Again using Proposition 6.1, we know that the function-space topology on $\mathcal{B}(M)$ is appropriate: We need to show that as $p^{\prime}$ approaches $p, b_{p^{\prime}}$ approaches $b_{p}$ in a pointwise fashion. We will accomplish this by showing, for any point $x=\left(\rho_{x}, p_{x}\right) \in M$,

$$
\left|b_{p}(x)-b_{p^{\prime}}(x)\right| \leq a\left(\rho_{x}\right) d_{K}\left(p, p^{\prime}\right) .
$$

We will not need the exact nature of the distance function in $M$, except for making use of Lemma 6.4. Since $b_{p}(x)=\lim _{s \rightarrow \omega}\left(s-d\left(x,\left(\rho_{0}+s, p\right)\right)\right)=-\rho_{0}+$ $\lim _{\rho \rightarrow \omega-\rho_{0}}(\rho-d(x,(\rho, p)))$ and similarly for $b_{p^{\prime}}(x)$, what we need to show is for all $\rho$,

$$
\left|d(x,(\rho, p))-d\left(x,\left(\rho, p^{\prime}\right)\right)\right| \leq a\left(\rho_{x}\right) d_{K}\left(p, p^{\prime}\right) .
$$

We will show $d(x,(\rho, p)) \leq a\left(\rho_{x}\right) d_{K}\left(p, p^{\prime}\right)+d\left(x,\left(\rho, p^{\prime}\right)\right)$ by exhibiting a point $q \in K$ such that

$$
\begin{aligned}
d\left(x,\left(\rho_{x}, q\right)\right) & \leq a\left(\rho_{x}\right) d_{K}\left(p, p^{\prime}\right) \quad \text { and } \\
d\left(\left(\rho_{x}, q\right),(\rho, p)\right) & \leq d\left(x,\left(\rho, p^{\prime}\right)\right) .
\end{aligned}
$$

Reversing $p$ and $p^{\prime}$ will then establish $d\left(x,\left(\rho, p^{\prime}\right)\right) \leq a\left(\rho_{x}\right) d_{K}\left(p, p^{\prime}\right)+d(x,(\rho, p))$, and we will be done.

Clearly, $d\left(x,\left(\rho_{x}, q\right)\right)=d\left(\left(\rho_{x}, p_{x}\right),\left(\rho_{x}, q\right)\right) \leq a\left(\rho_{x}\right) d_{K}\left(p_{x}, q\right)$ (just consider the constant- $\rho$ curve between the points); we still need $d_{K}\left(p_{x}, q\right) \leq d_{K}\left(p, p^{\prime}\right)$. In order to arrive at $d\left(\left(\rho_{x}, q\right),(\rho, p)\right) \leq d\left(\left(\rho_{x}, p_{x}\right),\left(\rho, p^{\prime}\right)\right)$, Lemma 6.4 says that all we need to do is to ensure $d_{K}(q, p) \leq d_{K}\left(p_{x}, p^{\prime}\right)$. Thus, the problem reduces to finding $q \in K$ so that

$$
\begin{aligned}
d_{K}\left(p_{x}, q\right) & \leq d_{K}\left(p, p^{\prime}\right) \quad \text { and } \\
d_{K}(q, p) & \leq d_{K}\left(p_{x}, p^{\prime}\right) .
\end{aligned}
$$

Consider a minimizing geodesic $\sigma$ from $p_{x}$ to $p$, with $\sigma(0)=p_{x}$ and $\sigma(1)=p$; we will locate $q$ along $\sigma$. Let $l=d_{K}\left(p_{x}, p^{\prime}\right)$ and $\bar{l}=d_{K}\left(p, p^{\prime}\right)$. For each $t \in[0,1]$, let $l_{t}$ be the length of $\sigma$ from 0 to $t$ and $\bar{l}_{t}$ its length from $t$ to 1 ; then $l_{t}+\bar{l}_{t}=d_{K}\left(p_{x}, p\right) \leq l+\bar{l}$. If we can find $t$ so that $l_{t} \leq \bar{l}$ and $\bar{l}_{t} \leq l$, then $q=\sigma(t)$ will do the job.

If $L(\sigma) \geq \bar{l}$, then there is some point $\sigma(t)$ along $\sigma$ so that $l_{t}=\bar{l}$; we then have $\bar{l}_{t} \leq l$, and we are done. If, on the other hand, $L(\sigma)<\bar{l}$, then let $t=1$ : $l_{1}=L(\sigma)<\bar{l}$ and $\bar{l}_{1}=0$, and again we are done.

Showing $i$ is injective.

We must show that for $p \neq p^{\prime},\left[b_{p}\right] \neq\left[b_{p^{\prime}}\right]$, i.e., that $b_{p}$ and $b_{p^{\prime}}$ do not differ merely by a constant. We will evaluate $b_{p}-b_{p^{\prime}}$ on two points: $x_{0}=\left(\rho_{0}, p\right)$ and $x_{0}^{\prime}=\left(\rho_{0}, p^{\prime}\right)$.

It's fairly easy to evaluate $b_{p}\left(\left(\rho_{0}, q\right)\right)$ for any $q$ :

$$
\begin{aligned}
b_{p}\left(\left(\rho_{0}, q\right)\right) & =\lim _{s \rightarrow \omega-\rho_{0}}\left(s-d\left(\left(\rho_{0}, q\right),\left(\rho_{0}+s, p\right)\right)\right) \\
& =-\rho_{0}+\lim _{s \rightarrow \omega}\left(s-\int_{\rho_{0}}^{s} \frac{1}{A\left(\theta_{s}^{q}\right)} d \rho\right),
\end{aligned}
$$


where $A(\theta)=\sqrt{1-\left(\frac{a_{0} \sin \theta}{a(\rho)}\right)^{2}}$ for $a_{0}=a\left(\rho_{0}\right)$, and $\theta_{s}^{q}$ is the initial radial angle for a minimizing geodesic from $\left(\rho_{0}, q\right)$ to $(s, p)$. Note that we are justified in using $\int$ instead of the more general $\oint$, since a geodesic starting from $\rho=\rho_{0}$ cannot have an initial radial angle greater than $\pi / 2$, if it is to reach any points at all in $M^{+}$.

Also worth noting: (1) For any $\bar{\rho} \in\left(\rho_{0}, \omega\right), I_{\rho_{0}}^{\bar{\rho}}$ is increasing (and continuous) on $[0, \pi / 2)$, with $I_{\rho_{0}}^{\bar{\rho}}(0)=0$ and $I_{\rho_{0}}^{\bar{\rho}}(\pi / 2)=\infty$. (2) For any $q \in K, \theta_{s}^{q}$ is decreasing in $s$ : For any $s<\omega, d_{K}(q, p)=I_{\rho_{0}}^{s}\left(\theta_{s}^{q}\right)$, so $s_{1}<s_{2}$ implies $I_{\rho_{0}}^{s_{1}}\left(\theta_{s_{1}}^{q}\right)=d_{K}(q, p)=$ $I_{\rho_{0}}^{s_{2}}\left(\theta_{s_{2}}^{q}\right)>I_{\rho_{0}}^{s_{1}}\left(\theta_{s_{2}}^{q}\right)$, whence $\theta_{s_{1}}^{q}>\theta_{s_{2}}^{q}$. (3) For each $\bar{\rho}$, there is some $\bar{\theta}<\pi / 2$ such that $I_{\rho_{0}}^{\bar{\rho}}(\bar{\theta})=\operatorname{diam}(K)$ : Just pick $\bar{p}$ and $\bar{q}$ so that $d_{K}(\bar{q}, \bar{p})$ realizes the diameter of $K ; \bar{\theta}=\theta \overline{\bar{q}}$ for that choice of $\bar{p}$. Then for any choice of $p$ and $q, \theta \frac{q}{\bar{\rho}} \leq \bar{\theta}$ (since $\left.I_{\rho_{0}}^{\bar{\rho}}\left(\theta_{\bar{\rho}}^{q}\right)=d(q, p) \leq \operatorname{diam}(K)=I_{\rho_{0}}^{\bar{\rho}}(\bar{\theta})\right)$.

We clearly have $\theta_{s}^{p}=0$ for all $s$, so $A\left(\theta_{s}^{p}\right)=1$ (we could, of course, just note $b_{p}\left(\left(\rho_{0}, p\right)\right)=0$, but it is convenient to treat it this way). Thus,

$$
\left(b_{p}-b_{p^{\prime}}\right)\left(x_{0}\right)=\lim _{s \rightarrow \omega} \int_{\rho_{0}}^{s}\left(\frac{1}{A\left(\theta_{s}^{p^{\prime}}\right)}-1\right) d \rho .
$$

Using $\frac{1}{\sqrt{1-x}}-1=\frac{x}{\sqrt{1-x}(1+\sqrt{1-x})}$, we get (writing $A_{s}$ for $A\left(\theta_{s}^{p^{\prime}}\right)$ ),

$$
\left(b_{p}-b_{p^{\prime}}\right)\left(x_{0}\right)=\lim _{s \rightarrow \omega}\left(a_{0}^{2} \sin ^{2} \theta_{s}^{p^{\prime}} \int_{\rho_{0}}^{s} \frac{d \rho}{a(\rho)^{2} A_{s}\left(1+A_{s}\right)}\right) .
$$

By Lemma 6.5, we can express the distance between $p$ and $p^{\prime}$ in terms of a minimizing geodesic from $x_{0}$ to $\left(s, p^{\prime}\right)$ :

$$
d_{K}\left(p, p^{\prime}\right)=a_{0} \sin \theta_{s}^{p^{\prime}} \int_{\rho_{0}}^{s} \frac{d \rho}{a(\rho)^{2} A_{s}} .
$$

Solving for $a_{0} \sin \theta_{s}^{p^{\prime}}$ and using that in the expression above, we get

$$
\left(b_{p}-b_{p^{\prime}}\right)\left(x_{0}\right)=d_{K}\left(p, p^{\prime}\right)^{2} \lim _{s \rightarrow \omega} \frac{\int_{\rho_{0}}^{s} \frac{d \rho}{a(\rho)^{2} A_{s}\left(1+A_{s}\right)}}{\left(\int_{\rho_{0}}^{s} \frac{d \rho}{a(\rho)^{2} A_{s}}\right)^{2}} .
$$

Since $a(\rho) \geq a\left(\rho_{0}\right)$, we have $1 \geq A_{s} \geq \cos \theta_{s}^{p^{\prime}}$, yielding

$$
\begin{aligned}
\left(b_{p}-b_{p^{\prime}}\right)\left(x_{0}\right) & \geq d_{K}\left(p, p^{\prime}\right)^{2} \lim _{s \rightarrow \omega} \frac{\frac{1}{2} \int_{\rho_{0}}^{s} \frac{d \rho}{a(\rho)^{2}}}{\left(\int_{\rho_{0}}^{s} \frac{d \rho}{a(\rho)^{2}}\right)^{2} \sec ^{2} \theta_{s}^{p^{\prime}}} \\
& =d_{K}\left(p, p^{\prime}\right)^{2} \frac{\cos ^{2} \theta_{\omega}^{p^{\prime}}}{2 \int_{\rho_{0}}^{\omega} \frac{d \rho}{a(\rho)^{2}}} .
\end{aligned}
$$

Pick some $\bar{\rho}$ with $\rho_{0}<\bar{\rho}<\omega$. As in (3) above, there is some maximum value $\bar{\theta}$ to $\theta_{\bar{\rho}}^{q}$ for all $q \in K, \bar{\theta}<\pi / 2$; note that $\theta_{\omega}^{p^{\prime}} \leq \theta_{\bar{\rho}}^{p^{\prime}}\left(\theta_{s}^{q}\right.$ is decreasing in $\left.s\right)$. With $\int_{\rho_{0}}^{\omega} 1 / a(\rho)^{2} d \rho=I<\infty$, this gives us

$$
\left(b_{p}-b_{p^{\prime}}\right)\left(x_{0}\right) \geq \frac{\cos ^{2} \bar{\theta}}{2 I} d_{K}\left(p^{\prime}, p\right)^{2} .
$$


Reversing $p$ and $p^{\prime}$ gives us

$$
\left(b_{p^{\prime}}-b_{p}\right)\left(x_{0}^{\prime}\right) \geq \frac{\cos ^{2} \bar{\theta}}{2 I} d_{K}\left(p, p^{\prime}\right)^{2}
$$

or

$$
\left(b_{p}-b_{p^{\prime}}\right)\left(x_{0}^{\prime}\right) \leq-\frac{\cos ^{2} \bar{\theta}}{2 I} d_{K}\left(p, p^{\prime}\right)^{2} .
$$

It follows that for $p \neq p^{\prime}, b_{p}-b_{p^{\prime}}$ is non-constant.

Showing $i^{-1}$ is continuous.

We must show that given any sequence $\left\{\left[b_{p_{n}}\right]\right\}$ converging to $\left[b_{p}\right]$ in $B_{\omega}$, that $\left\{p_{n}\right\}$ converges to $p$. Recall that in the function-space topology on $\mathcal{L}_{1}(M)$, we have a continuous cross-section $\zeta: \mathcal{L}_{1}(M) / \mathbb{R} \rightarrow \mathcal{L}_{1}(M)$ given by $\zeta:[f] \mapsto f-f(\bar{x})$, where $\bar{x}$ is any point we care to fix in $M$. It follows that $\left\{\zeta\left(\left[b_{p_{n}}\right]\right)\right\}$ converges to $\zeta\left(\left[b_{p}\right]\right)$, i.e., $\left\{b_{p_{n}}-b_{p_{n}}(\bar{x})\right\}$ converges pointwise to $b_{p}-b_{p}(\bar{x})$. In particular, we may choose to let $\bar{x}=x_{0}=\left(\rho_{0}, p\right)$. This gives us $\left\{b_{p_{n}}-b_{p_{n}}\left(x_{0}\right)\right\}$ converging pointwise to $b_{p}-b_{p}\left(x_{0}\right)$. Apply this to any point $y_{0}=\left(\rho_{0}, q\right):\left\{b_{p_{n}}\left(y_{0}\right)-b_{p_{n}}\left(x_{0}\right)-\left(b_{p}\left(y_{0}\right)-b_{p}\left(x_{0}\right)\right)\right\}$ converges to 0 . (Actually, $b_{p}\left(x_{0}\right)=0$, but preserving the symmetry of expression is more clarifying than using this fact.)

Suppose that $\left\{p_{n}\right\}$ fails to converge to $p$; then there is a subsequence, which we will also call $\left\{p_{n}\right\}$, such that for all $n, d_{K}\left(p_{n}, p\right) \geq \delta$ for some $\delta>0$. Furthermore, we can find a further subsequence - still denoted $\left\{p_{n}\right\}$-converging to some point $q \neq p$. Applying the inequalities at the end of the last section yields $\left(b_{p}-b_{p_{n}}\right)\left(x_{0}\right) \geq$ $\left(\cos ^{2} \bar{\theta} /(2 I)\right) d_{K}\left(p_{n}, p\right)^{2}$ and $\left(b_{p_{n}}-b_{p}\right)\left(x_{n}\right) \geq\left(\cos ^{2} \bar{\theta} /(2 I)\right) d_{K}\left(p_{n}, p\right)^{2}$, where $x_{n}=$ $\left(\rho_{0}, p_{n}\right)$. Therefore, for some $\epsilon>0$, we have (for $n$ sufficiently large)

$$
\begin{aligned}
b_{p}\left(x_{0}\right)-b_{p_{n}}\left(x_{0}\right) & \geq 2 \epsilon \quad \text { and } \\
b_{p_{n}}\left(x_{n}\right)-b_{p}\left(x_{n}\right) & \geq 2 \epsilon .
\end{aligned}
$$

Let $y_{0}=\left(\rho_{0}, q\right)$, which is $\lim x_{n}$ for our subsequence; then, as Busemann functions are Lipschitz-1, that last inequality yields (for $n$ sufficiently large that $d\left(x_{n}, y_{0}\right)<$ $\epsilon / 2)$

$$
b_{p_{n}}\left(y_{0}\right)-b_{p}\left(y_{0}\right) \geq \epsilon .
$$

That gives us $b_{p_{n}}\left(y_{0}\right)-b_{p_{n}}\left(x_{0}\right)-\left(b_{p}\left(y_{0}\right)-b_{p}\left(x_{0}\right)\right) \geq 3 \epsilon$, contradicting $\left\{b_{p_{n}}\left(y_{0}\right)-\right.$ $\left.b_{p_{n}}\left(x_{0}\right)-\left(b_{p}\left(y_{0}\right)-b_{p}\left(x_{0}\right)\right)\right\}$ converging to 0 . It follows that $\left\{p_{n}\right\}$ must converge to $p$.

Theorem 6 of [H3] establishes the causal/chronological nature of the lines forming the cones: A line-element of the cone is the $\mathbb{R}$-orbit of a Busemann function $b_{c}$; if $c$ is a curve of finite length, the line is timelike $\left(b_{c}+t \ll b_{c}+t^{\prime}\right.$ for $\left.t<t^{\prime}\right)$, while if $c$ is of infinite length, the line is null $\left(b_{c}+t \prec b_{c}+t^{\prime}\right.$ for $t<t^{\prime}$, but no $\ll$ relations $)$.

Corollary 6.6. Let $V$ be a spacetime with topology $\mathbb{R}^{1} \times\left(r_{\min }, r_{\max }\right) \times \mathbb{S}^{2}\left(r_{\min }>0\right)$ and metric $g=-f(r) d t^{2}+(1 / f(r)) d r^{2}+r^{2} d \mathbb{S}^{2}$, where $f$ is some positive function on $\left(r_{\min }, r_{\max }\right)$ and $d \mathbb{S}^{2}$ is the round metric on the sphere. Let $\phi(r)=r^{2} / f(r)$. If $\phi$ has minimum value at $r_{0}$ and is decreasing on $\left(r_{\min }, r_{0}\right)$ and increasing on $\left(r_{0}, r_{\max }\right)$, then the future-completion of $V$ (in the future chronological topology) is Hausdorff, and the future causal boundary consists of two cones on $\mathbb{S}^{2}$-corresponding to going out to infinity in the $r=r_{\min }$ and $r=r_{\max }$ directions, respectively-conjoined 
at their common vertex. The $r_{\max }$ cone is null if $\int_{r_{0}}^{r_{\max }} 1 / f(r) d r=\infty$, otherwise timelike; similarly for the $r_{\min }$ cone.

If $V=\mathbb{R}^{1} \times\left(0, r_{\max }\right) \times \mathbb{S}^{2}$ with metric, $f, \phi$, and $r_{0}$ all as above, then the result changes only in that the $r_{\min }$ cone is replaced by a single line-null if $\int_{0}^{r_{0}} 1 / f(r) d r=$ $\infty$, otherwise timelike.

Proof. The metric on $V$ is conformal to $\bar{g}=-d t^{2}+(1 / f(r))^{2} d r^{2}+\left(r^{2} / f(r)\right) d \mathbb{S}^{2}$. If we define $\rho$ by $d \rho=d r / f(r)$, then we have the situation prescribed by the hypotheses of Theorem 6.2 , with $\alpha=\int_{r_{\min }}^{r_{0}} 1 / f(r) d r, \omega=\int_{r_{0}}^{r_{\max }} 1 / f(r) d r$, and $a(\rho)=\sqrt{\phi(r)}$. We have $\int_{\rho_{0}}^{\omega} \frac{1}{a(\rho)^{2}} d \rho=\int_{r_{0}}^{r_{\max }} \frac{1}{\phi(r)} \frac{d r}{f(r)}=\int_{r_{0}}^{r_{\max }} \frac{1}{r^{2}} d r=1 / r_{0}-$ $1 / r_{\max }$, which is necessarily finite; similarly for the other endpoint if $r_{\min }>0$. If $r_{\min }=0$, then $\int_{\alpha}^{\rho_{0}} 1 / a(\rho)^{2} d \rho=\int_{0}^{r_{0}} 1 / r^{2} d r=\infty$.

We will explore applications of Corollary 6.6 in a discussion subsection. Before that, we present an extension of Theorem 6.2 to more general warping functions; though it appears no classical spacetimes are examples of this extension, it lends itself to a useful perspective.

Theorem 6.7. Let $M$ be a Riemannian manifold expressible as a warped product with a compact manifold, $(\alpha, \omega) \times{ }_{a} K$, metric $d \rho^{2}+a(\rho)^{2} j_{k}$. Suppose for some $\rho_{-}<\rho_{+}$in $(\alpha, \omega)$, a is decreasing on $\left(\alpha, \rho_{-}\right)$and increasing on $\left(\rho_{+}, \omega\right)$. Then the same conclusions from Theorem 6.2 apply: The Busemann boundary of $M$ is Hausdorff and consists of spaces $B_{\iota}$ attaching at $\{\iota\} \times K, \iota=\alpha, \omega$, with $B_{\iota}$ a point if $\left|\int_{\rho_{+}}^{\iota} 1 / a(\rho)^{2} d \rho\right|=\infty$ and $B_{\iota} \cong K$ otherwise. The future causal boundary of $V=\mathbb{L}^{1} \times(\alpha, \omega) \times{ }_{a} K$ is a pair of conjoined cones over $B_{\alpha}$ and $B_{\omega}$, the respective cone being null if the endpoint $(\alpha$ or $\omega)$ is infinite, otherwise timelike.

Proof. All that was done in Theorem 6.2 with respect to $\left(\rho_{0}, \omega\right)$ can be done here with $\left(\rho_{+}, \omega\right)$.

An alternative view: $\left[\rho_{-}, \rho_{+}\right]$can be decomposed into a finite number of subintervals $\bigcup_{1=1}^{2 m}\left[\rho_{i-1}, \rho_{i}\right]\left(\rho_{0}=\rho_{-}, \rho_{2 m}=\rho_{+}\right)$on each of which $a$ is monotonic. We can apply Thereom 6.2 separately to $\left(\alpha, \rho_{1}\right) \times_{a} K$, to $\left(\rho_{1}, \rho_{3}\right) \times_{a} K$, and so on up through $\left(\rho_{2 m-1}, \omega\right) \times{ }_{a} K$. The spacetimes $V_{i}=\mathbb{L}^{1} \times\left(\rho_{2 i-1}, \rho_{2 i+1}\right) \times{ }_{a} K$ each have, as future causal boundary, timelike cones over two copies of $K$, one each at $\left\{\rho_{2 i-1}\right\} \times K$ and $\left\{\rho_{2 i+1}\right\} \times K$ (and similarly, mutatis mutandis, for $\mathbb{L}^{1} \times\left(\alpha, \rho_{1}\right) \times K$ and $\left.\mathbb{L}^{1} \times\left(\rho_{2 m-1}, \omega\right) \times{ }_{a} K\right)$. Thus, the future-completions $V_{i}^{+}$fit together quite neatly, boundaries all matching in topology and causality. The union of all those is $V^{+}$.

Corollary 6.8. Let $V$ be a spacetime with toplogy $\mathbb{R}^{1} \times\left(r_{\min }, r_{\max }\right) \times \mathbb{S}^{2}\left(r_{\min }>0\right)$ and metric $-f(r) d t^{2}+(1 / f(r)) d r^{2}+r^{2} d \mathbb{S}^{2}$ for $f$ a positive function on $\left(r_{\min }, r_{\max }\right)$. If $r^{2} / f(r)$ is decreasing on some interval $\left(r_{\min }, r_{-}\right)$and increasing on some interval $\left(r_{+}, r_{\max }\right)$, then the future-completion of $V$ is Hausdorff, and the future causal boundary consists of two conjoined cones on $\mathbb{S}^{2}$; the cone at $r_{\max }$ is null if $\int_{r_{0}}^{r_{\max }} 1 / f(r) d r=\infty$, otherwise timelike (and similarly for the cone at $r_{\min }$ ).

If $V=\mathbb{R} \times\left(0, r_{\max }\right) \times \mathbb{S}^{2}$ with metric as above, then the result changes only in that the $r_{\min }$ cone is replaced by a single line-null if $\int_{0}^{r_{-}} 1 / f(r) d r=\infty$, otherwise timelike. 
Further generalizations are possible. For instance, the same results as in Theorems 6.2 or 6.7 can be proved with $a$ having the property that for any $\rho$ in its domain, there exist $\rho^{-}$and $\rho^{+}$in the domain with $\rho^{-}<\rho<\rho^{+}$such that for any $s<\rho^{-}, a(s)>a\left(\rho^{-}\right)$and for any $s>\rho^{+}, a(s)>a\left(\rho^{+}\right)$(one proves that lengths are realized by geodesics in the same manner as in Theorem 6.2, and sections I and IIa follow nearly the same as before; IIb is handled by observing that a geodesic which starts at a point where $a$ has its global minimum can be parametrized by $\rho$ on its entire length). Of far more practical use are versions where $a$ is monotonic. This is much more difficult in general; a version for monotonic $a$ in which $a$ approaches 0 is discussed in some detail in the discussion section, at least for the case of $\int 1 / a(\rho)^{2} d \rho=\infty$, as this is important for Reissner-Nordström.

\section{Discussion: Three classical spacetimes.}

A number of classical spacetimes have sections that have the form specified in Corollary 6.6. We will briefly examine three classes of such spacetimes and also look at what is known by $[\mathrm{H} 4]$ about the other sections of those spacetimes and how the sections fit together. The object in all of these examinations is not to discover previously unknown properties, as most of these boundaries are already widely known or at least suspected; rather, the point is to show how the methods of this paper and of [H4] can be easily applied. But it should be noted that what is established here are universal results - in the topological sense of [H2] for spacelike boundaries and at least in the chronological sense of [H1] for timelike and null boundaries. This tells us that any other completions must be derived from these natural ones, such as by quotients.

Some of the boundaries we will exhibit are interior boundaries, in the sense of being boundaries of sections of the spacetime that, in the maximally extended model, have another section on the other side, and the boundaries exemplified here are illustrative for showing how the different sections fit together. These are not intended as proofs of the global structure of these spacetimes, and we are relying on the reader's prior understanding of the global nature; the point is to show how these boundary results fit into the general picture.

\section{Schwarzschild.}

As mentioned above, the Schwarzschild metric, representing a spherically symmetric, uncharged black hole of mass $m>0$ in a vacuum spacetime which is asymptotically flat, is

$$
d s^{2}=-\left(1-\frac{2 m}{r}\right) d t^{2}+\left(1-\frac{2 m}{r}\right)^{-1} d r^{2}+r^{2} d \mathbb{S}^{2}
$$

where $d \mathbb{S}^{2}$ denotes the round metric on the sphere. This has the form of Corollary 6.6 for $f(r)=1-\frac{2 m}{r}$, which is positive for $r$ in $(2 m, \infty)$. Thus, we are looking here at external Schwarzschild (that is to say, external to the event horizon), $r>2 m$; the manifold is $\mathbf{S} c h_{\text {ext }}=\mathbb{R} \times(2 m, \infty) \times \mathbb{S}^{2}$. For $\phi(r)=r^{2} / f(r)$ we easily note that $\phi$ has a minimum at $r=3 \mathrm{~m}$, with $\phi$ monotone on either side of that minimum. This puts Corollary 6.6 into play. We have $\int_{3 m}^{\infty} 1 / f(r) d r=\infty$ (just note that $f(r)<1$ ) and $\int_{2 m}^{3 m} 1 / f(r) d r=\infty$ (for $r$ close to $2 m, 1 / f(r)=r /(r-2 m)$ behaves like $2 m /(r-2 m))$. Therefore $\partial^{+}\left(\mathbf{S c h}_{\text {ext }}\right)$ is a pair of conjoined null cones (cones on $\mathbb{S}^{2}$ ), one at infinity and one at the event horizon; the conjunction, a single point, may reasonably be labled $i^{+}$, future timelike infinity. 
Of course, we fully expect a Minkowski-like future causal boundary for Schwarzschild, and that's the null cone at infinity - but what's this other boundary at $r=2 m$ ? That is an artifact of cutting off the full Schwarzschild spacetime at the event horizon; all it does is tell us that we cut along a null cone to obtain the exterior sector. What about looking at the full spacetime - or, at least, other sectors? (See [HE] for a thorough discussion of maximally extended Schwarzschild.)

Let us first examine interior Schwarzschild: With $0<r<2 m$, the metric is perhaps more readily understood as

$$
d s^{2}=-\left(\frac{2 m}{r}-1\right)^{-1} d r^{2}+\left(\frac{2 m}{r}-1\right) d t^{2}+r^{2} d \mathbb{S}^{2}
$$

on a manifold $\mathbf{S} \mathbf{c h}$ int $=(0,2 m) \times \mathbb{R} \times \mathbb{S}^{2}$, with the time orientation having 0 as the future endpoint of the first factor (particles fall inward). This is analyzed in section 5.2 of [H2]: $\mathbf{S c h}_{\text {int }}$ is conformal to a warped product, metric $-d r^{2}+\left(\frac{2 m}{r}-1\right)^{2} d t^{2}+$ $r^{2}\left(\frac{2 m}{r}-1\right) d \mathbb{S}^{2}$. We apply Proposition 5.2 in [H2]: The spacelike factors $\mathbb{R}$ and $\mathbb{S}^{2}$ are both complete and the integrals of the warping functions, near the future endpoint of $(0,2 m)$, yield $\int_{0}^{\epsilon}\left(\frac{2 m}{r}-1\right)^{-1} d r<\infty$ and $\int_{0}^{\epsilon} r^{-1}\left(\frac{2 m}{r}-1\right)^{-\frac{1}{2}} d r<\infty$. This implies $\partial^{+}\left(\mathbf{S c h}_{\text {int }}\right)$ is spacelike and is the product of the spacelike factors of the spacetime, $\mathbb{R} \times \mathbb{S}^{2}$.

But this analysis tells us nothing of how $\mathbf{S c h}_{\text {int }}$ and $\mathbf{S} \mathbf{c h}$ ext fit together. Since the exterior portion joins the interior portion at $r=2 m$, which is at the past endpoint of the timelike factor in $\mathbf{S c h}_{\text {int }}$, it is $\partial^{-}(\mathbf{S} \mathbf{c h}$ int $)$ we need to look at. When we look at the integrals of the warping functions near the other endpoint, we discover $\int_{2 m-\epsilon}^{2 m}\left(\frac{2 m}{r}-1\right)^{-1} d r=\infty$ and $\int_{2 m-\epsilon}^{2 m} r^{-1}\left(\frac{2 m}{r}-1\right)^{-\frac{1}{2}} d r<\infty$. Proposition 5.2 of [H2] doesn't apply in this case; but its extension in Proposition 3.5(b) of [H4] does, telling us that, with a non-compact spacelike factor having an infinite integral for its warping function, $\partial^{-}\left(\mathbf{S c h}_{\text {int }}\right)$ has null-related elements.

That proposition concerns the causal boundary of a spacetime of the form $V=$ $(a, b) \times_{f_{1}} K_{1} \times \cdots \times_{f_{k}} K_{k} \times{ }_{f_{k+1}} K_{k+1} \times \cdots \times_{f_{m}} K_{m}$, where each $f_{i}:(a, b) \rightarrow \mathbb{R}$ is a positive function with $\int_{b^{-}}^{b} f_{i}^{-1 / 2}<\infty$ for $i \leq k$ and $\int_{b^{-}}^{b} f_{i}^{-1 / 2}=\infty$ for $i>k$, each $K_{i}$ is a manifold with a complete Riemannian metric $h_{i}$, and the spacetime metric is $-d t^{2}+\sum_{i} f_{i}(t) h_{i}$. If $K_{i}$ is non-compact for some $i>k$, the proof there shows how to construct a null line in $\partial^{+}(V)$ out of any choice of point $x^{0} \in K^{0}=K_{1} \times \cdots \times K_{k}$ and the Busemann function for any infinite-length ray $c_{i} \in K_{i}$. This can be amplified a bit in the case that $m=k+1: \partial^{+}(V)=\left(K^{0} \times \mathcal{B}\left(K_{k+1}\right) \times \mathbb{R}\right) \cup K^{0}$, where $\mathcal{B}\left(K_{k+1}\right)$ is the set of equivalence classes of finite Busemann functions on $K_{k+1}$, the $\mathbb{R}$ factor is a set of null lines on $K^{0} \times \mathcal{B}\left(K_{k+1}\right)$, and those lines all converge to a spacelike image of $K^{0}$; in effect, this is a null cylinder on $K^{0} \times \mathcal{B}\left(K_{k+1}\right)$, capped off by squeezing the $\mathcal{B}\left(K_{k+1}\right)$ factor to a point. (The picture is more complex when $m>k+1)$.

We can apply this for $\partial^{-}\left(\mathbf{S c h}_{\text {int }}\right)$ (time-reversing the result above): The warping function for the $\mathbb{S}^{2}$ factor yields a finite integral, that for the $\mathbb{R}$ factor an infinite integral. There are exactly two classes of finite Busemann functions on $\mathbb{R}$, associated with the two rays, one for each end of $\mathbb{R}$. We conclude that $\partial^{-}\left(\mathbf{S c h}_{\text {int }}\right)$ consists of a pair of null cylinders on $\mathbb{S}^{2}$, one for $t=\infty$ and one for $t=-\infty$, conjoined in a spacelike $\mathbb{S}^{2}$, much like an extended $i^{-}$. The $t=\infty$ null cylinder in $\partial^{-}\left(\mathbf{S c h}_{\text {int }}\right)$ is identified with the $r=2 m$ null cone in $\partial^{+}\left(\mathbf{S c h}_{\text {ext }}\right)$ (external time coordinate is 
infinite for passage through the event horizon); as it is only the null lines in either cylinder or cone which are identified, there is no problem with the vertex of the cone or the cap on the cylinder. Thus do $\mathbf{S} \mathbf{c h}$ ext and $\mathbf{S} \mathbf{c h}$ int fit together across the evident topological and causal match of their boundary components (though it takes other considerations to guarantee that we have a geometric match).

The $t=-\infty$ null cylinder in $\partial^{-}\left(\mathbf{S c h}_{\text {int }}\right)$ fits with a corresponding null cone in the future causal boundary of $\mathbf{S} \mathbf{c h}_{\text {alt }}$, the "alternate universe" sector of maximally extended Schwarzschild (isometric with $\mathbf{S c h}_{\text {ext }}$, though $t$-reversed). The $r=2 m$ null cones in $\partial^{-}\left(\mathbf{S c h}_{\text {ext }}\right)$ and $\partial^{-}\left(\mathbf{S c h}_{\text {alt }}\right)$ fit with the two null cylinders in the future causal boundary of $\mathbf{S c h}_{\mathrm{whh}}$, the "white hole" sector of maximal Schwarzschild (isometric with a time-reversal of $\mathbf{S} \mathbf{h}_{\text {int }}$ ). The two spacelike $\mathbb{S}^{2}$ caps-one from $\partial^{-}\left(\mathbf{S c h}_{\text {int }}\right)$ and one from $\partial^{+}\left(\mathbf{S c h}_{\mathrm{whh}}\right)$-become identified as a sphere in the spacetime at the juncture of the four sectors. Thus, the entire causal boundary of $\mathbf{S c h}=\mathbf{S c h}_{\text {ext }} \cup \mathbf{S c h}_{\text {int }} \cup \mathbf{S c h}_{\text {alt }} \cup \mathbf{S c h}_{\text {whh }}$ consists of two null cones in the future boundary joining a future singularity of $\mathbb{R} \times \mathbb{S}^{2}$ (with the joins being points, $i^{+}$and $i_{\text {alt }}^{+}$, closing off either end of that cylinder) and the past mirror of all that.

Schwarzschild-de Sitter.

Schwarzschild-de Sitter models a spherically symmetric, uncharged, black hole of mass $m>0$ in a vacuum spacetime with cosmological constant $\Lambda>0$, asymptotically like de Sitter space (a universe with accelerating expansion); see, for instance $[\mathrm{R}]$. Its metric is

$$
d s^{2}=-\left(1-\frac{2 m}{r}-\frac{\Lambda}{3} r^{2}\right) d t^{2}+\left(1-\frac{2 m}{r}-\frac{\Lambda}{3} r^{2}\right)^{-1} d r^{2}+r^{2} d \mathbb{S}^{2}
$$

this gives us $f(r)=1-\frac{2 m}{r}-\frac{\Lambda}{3} r^{2}$, which has an interval for which it is positive if and only if $\Lambda<1 /\left(9 m^{2}\right)$. Assuming, then, that $0<\Lambda<1 /\left(9 m^{2}\right)$, we have $f>0$ on an interval $\left(r_{-}, r_{+}\right)$, where $0<r_{-}<3 m<r_{+}$. This yields $\phi(r)=r^{2} / f(r)$ having a minimum at $r=3 m$ and going to infinity monotonically at both $r_{-}$and $r_{+}$. Thus, we can apply Corollary 6.6 to what we may perhaps call medial Schwarzschild-de Sitter, $\mathbf{S d} \mathbf{S}_{\text {med }}=\mathbb{R} \times\left(r_{-}, r_{+}\right) \times \mathbb{S}^{2}$ : Since $\int_{r_{-}}^{3 m} 1 / f(r) d r=\int_{3 m}^{r_{+}} 1 / f(r) d r=\infty$ (just note that for some constant $A, 1 / f(r)=r\left(r-2 m-(\Lambda / 3) r^{3}\right)^{-1}$ behaves like $A\left(r-r_{-}\right)^{-1}$ for $r$ close to $r_{-}$, and so on), we get $\partial^{+}\left(\mathbf{S d} \mathbf{S}_{\text {med }}\right)$ is a pair of conjoined null cones on $\mathbb{S}^{2}$, one each at $r=r_{-}$and $r=r_{+}$and meeting in a point which we'll call $i^{+}$by analogy with Schwarzschild.

As with Schwarzschild, we can examine the causal boundary of other sectors, where $f<0$, by using Proposition 3.5(b) of [H4]. For interior Schwarzschild-de Sitter, $\mathbf{S d S}_{\text {int }}=\left(0, r_{-}\right) \times \mathbb{R} \times \mathbb{S}^{2}$, we get the same as for interior Schwarzschild: $\partial^{+}\left(\mathbf{S d S}_{\text {int }}\right)$ is a spacelike cylinder $\mathbb{R} \times \mathbb{S}^{2}$, the singularity at $r=0$, while $\partial^{-}\left(\mathbf{S d S}_{\text {int }}\right)$ is a pair of null cylinders on $\mathbb{S}^{2}$, one each at $t=\infty$ and $t=-\infty$, sharing a mutual cap of a spacelike $\mathbb{S}^{2}$; and these all match up with the boundaries of $\mathbf{S d} \mathbf{S}_{\text {med }}$ just as the boundaries of interior and exterior Schwarzschild match up. We also have exterior Schwarzschild-de Sitter, $\mathbf{S d S}_{\text {ext }}=\left(r_{+}, \infty\right) \times \mathbb{R} \times \mathbb{S}^{2}$, which behaves very similarly: $\partial^{+}\left(\mathbf{S d S}_{\text {ext }}\right)$ is the de Sitter-like boundary at infinity, a spacelike cylinder $\mathbb{R} \times \mathbb{S}^{2}$ at $r=\infty$, while $\partial^{-}\left(\mathbf{S d S}_{\text {ext }}\right)$ is a pair of null cylinders on $\mathbb{S}^{2}$ at $t=\infty$ and $t=-\infty$, meeting in a spacelike $\mathbb{S}^{2}$; these also join up with $\mathbf{S d} \mathbf{S}_{\text {med }}$ in a similar fashion, with the $t=\infty$ end of the cylinder in $\partial^{+}\left(\mathbf{S d S}_{\text {ext }}\right)$ joining to the point $i^{+}$ 
in $\partial^{+}\left(\mathbf{S d} \mathbf{S}_{\mathrm{med}}\right)$, and the null cylinder in $\partial^{-}\left(\mathbf{S} \mathbf{d} \mathbf{S}_{\mathrm{ext}}\right)$ at $t=\infty$ matching the null cone in $\partial^{+}\left(\mathbf{S d} \mathbf{S}_{\text {med }}\right)$ at $r=r_{+}$.

This still leaves the $t=-\infty$ null cylinder in $\partial^{-}\left(\mathbf{S d S}_{\text {ext }}\right)$, which analysis shows not to be at geometric infinity (for instance, radial null geodesics are affinely parametrized by $r$, so we should expect continuance beyond $\left.r=r_{+}\right)$: In maximally extended Schwarzschild-de Sitter (see [GiH]), another (t-reversed) copy of SdS $\mathbf{S}_{\text {med }}$ attaches to that cylinder, via that copy's $r=r_{+}$null cone in $\partial^{+}\left(\mathbf{S d S}_{\text {med }}\right)$; likewise, the $r=r_{-}$cone attaches to another copy of $\mathbf{S d S}_{\text {int }}$ via one cylinder in $\partial^{-}\left(\mathbf{S} \mathbf{d} \mathbf{S}_{\text {int }}\right)$, and the whole process repeats at the other cone in that boundary, yielding further copies of $\mathbf{S d} \mathbf{S}_{\text {med }}$, then $\mathbf{S d} \mathbf{S}_{\text {ext }}$, then $\mathbf{S d} \mathbf{S}_{\text {med }}, \mathbf{S d} \mathbf{S}_{\text {int }}$, and so forth. Looking back at the original $\mathbf{S d} \mathbf{S}_{\text {int }}$, we find yet another copy of $\mathbf{S d} \mathbf{S}_{\text {med }}$ attaching (just as $\mathbf{S} \mathbf{c h}_{\text {alt }}$ attaches to $\mathbf{S} \mathbf{c h}$ int), along with a corresponding string of further

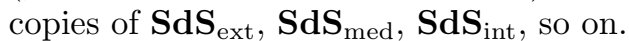

And just as $\mathbf{S} \mathbf{c h}_{\text {whh }}$ attaches to $\mathbf{S} \mathbf{c h}$ ext and $\mathbf{S} \mathbf{c h}$ alt , the maximal extension also has a time-reversed copy of $\mathbf{S d} \mathbf{S}_{\text {int }}$, which we may call $\mathbf{S d} \mathbf{S}_{\text {whh }}$, attaching to neighboring copies of $\mathbf{S d} \mathbf{S}_{\text {med }}$ : each of the two null cylinders in $\partial^{+}\left(\mathbf{S d} \mathbf{S}_{\mathrm{whh}}\right)$ attaching to one of the null cones in each of the copies of $\partial^{-}\left(\mathbf{S d} \mathbf{S}_{\text {med }}\right)$. Thus, the entire future causal boundary of maximal SdS consists of a string of spacelike cylinders $\mathbb{R} \times \mathbb{S}^{2}$ (alternating as spatially extended sigularities and spatially extended infinities) joined together with points $i^{+}$which close off the cylinders; the past causal boundary is the mirror of that with no connection to the future causal boundary.

One may also consider Schwarzschild-Anti-de Sitter space, which is the same metric but with $\Lambda<0$, i.e., a negative cosmological constant and a spacetime which is asymptotically like anti-de Sitter space. We have $f>0$ on $\left(r_{0}, \infty\right)$ for some $r_{0}<2 m$, and $\phi(r)=r^{2} / f(r)$ has a minimum at $r=3 m$, going monotonically to inifinty at $r=r_{0}$ and monotonically to $3 /(-\Lambda)$ at $r=\infty$. Thus, $\mathbf{S} \mathbf{A d S} \mathbf{S}_{\text {ext }}=\mathbb{R} \times$ $\left(r_{0}, \infty\right) \times \mathbb{S}^{2}$ comes under the conditions of Corollary 6.6. We have $\int_{r_{0}}^{3 m} 1 / f(r) d r=$ $\infty$, but $\int_{3 m}^{\infty} 1 / f(r) d r<\infty\left(\right.$ as $1 / f(r)$ behaves like $r^{-2}$ for $r$ near $\left.\infty\right)$. This tells us $\partial^{+}\left(\mathbf{S A d S}_{\text {ext }}\right)$ has a null cone on $\mathbb{S}^{2}$ at $r=r_{0}$ but a timelike cone on $\mathbb{S}^{2}$ at $r=\infty$ (the two cones conjoined at the vertex $\left.i^{+}\right)$. The same is true for $\partial^{-}\left(\mathbf{S A d S} \mathbf{S}_{\text {ext }}\right)$, of course (with vertex $i^{-}$). It is common wisdom to combine timelike elements of the future and past causal boundaries, yielding a timelike suspension (cylinder closed at both ends) on $\mathbb{S}^{2}$ at $r=\infty$ serving duty (along with the respective null cones at $\left.r=r_{0}\right)$ for both $\partial^{+}\left(\mathbf{S} \mathbf{A d} \mathbf{S}_{\text {ext }}\right)$ and $\partial^{-}\left(\mathbf{S A d S}_{\text {ext }}\right)$. This timelike suspension on $\mathbb{S}^{2}$ lies at geometric infinity; there is no extension possible beyond it (null geodesics cannot extend beyond $r=\infty$; it is essentially the anti-de Sitter horizon at spatial infinity). But the $r=r_{0}$ null cone attaches, just as before, to a black-hole-like $\mathbf{S A d S}_{\text {int }}$ and white-hole-like $\mathbf{S} \mathbf{A d} \mathbf{S}_{\mathrm{whh}}$, with a ( $t$-reversed) $\mathbf{S} \mathbf{A d \mathbf { S } _ { \text { alt } }}$ attaching on the other boundaries of $\mathbf{S A d} \mathbf{S}_{\mathrm{int}}$ and $\mathbf{S A d} \mathbf{S}_{\mathrm{whh}}$.

This yields a total causal boundary for maximal SAdS (assuming the standard identification) as two timelike cyinders $\mathbb{R} \times \mathbb{S}^{2}$ (at infinity) joined to two spacelike cylinders $\mathbb{R} \times \mathbb{S}^{2}$ (singularities) at points $i^{ \pm}$and $i_{\text {alt }}^{ \pm}$, closing off the cylinders.

It should be noted that issues of identification of various parts of the future and past causal boundaries typically become important, and possibly controversial, when portions of the boundaries have timelike character. Some discussion of appropriate conditions for making identifications within the causal boundary construction can be found in $[\mathrm{S}]$. A somewhat different (but closely related) approach can be found in $[\mathrm{MR}]$ and $[\mathrm{F}]$. 


\section{Reissner-Nordström.}

Reissner-Nordström models a spherically symmetric black hole of mass $m>$ 0 and electric charge $q \neq 0$ in an otherwise empty, asymptotically flat universe (extensively described in [HE]). The metric is

$$
d s^{2}=-\left(1-\frac{2 m}{r}+\frac{q^{2}}{r^{2}}\right) d t^{2}+\left(1-\frac{2 m}{r}+\frac{q^{2}}{r^{2}}\right)^{-1} d r^{2}+r^{2} d \mathbb{S}^{2}
$$

i.e., $f(r)=1-\frac{2 m}{r}+\frac{q^{2}}{r^{2}}$. The analysis of $f$ depends on the relative sizes of $q$ and $m$.

First assume the undercharged case, $|q|<m$; then $f>0$ on two intervals $\left(0, r_{-}\right)$ and $\left(r_{+}, \infty\right)$, where $r_{ \pm}=m\left(1 \pm \sqrt{1-\left(\frac{q}{m}\right)^{2}}\right)$. We find $\phi(r)=r^{2} / f(r)$ has a local minimum at $r_{+}^{0}=\frac{3}{2} m\left(1+\sqrt{1-\left(\frac{2}{3} \frac{q}{m}\right)^{2}}\right)>r_{+}$and is monotonic on either side of $r_{+}^{0}$ within $\left(r^{+}, \infty\right)$. Thus, Corollary 6.6 applies to exterior undercharged ReissnerNordström $\mathbf{R N}_{\mathrm{ext}}^{\text {und }}=\mathbb{R} \times\left(r_{+}, \infty\right) \times \mathbb{S}^{2}$. We have both $\int_{r_{+}^{0}}^{\infty} 1 / f(r) d r=\infty$ and $\int_{r_{+}}^{r_{+}^{0}} 1 / f(r) d r=\infty$, so $\partial^{+}\left(\mathbf{R N}_{\mathrm{ext}}^{\text {und }}\right)$ consists of two null cones on $\mathbb{S}^{2}$ conjoined at the vertex $i^{+}$, with $\partial^{-}\left(\mathbf{R} \mathbf{N}_{\text {ext }}^{\text {und }}\right)$ analogous.

The medial portion $\mathbf{R} \mathbf{N}_{\text {med }}^{\text {und }}=\left(r_{-}, r_{+}\right) \times \mathbb{R} \times \mathbb{S}^{2}$ can be analyzed with the means in [H4]: The metric is conformal to the warped product metric $-d r^{2}+\left(-\left(\frac{q}{r}\right)^{2}+\right.$ $\left.\frac{2 m}{r}-1\right)^{2} d t^{2}+r^{2}\left(-\left(\frac{q}{r}\right)^{2}+\frac{2 m}{r}-1\right) d \mathbb{S}^{2}$; but in this case, the appropriate integrals of the warping function for the $\mathbb{R}$ factor are finite at both the future $\left(r_{-}\right)$and past $\left(r_{+}\right)$endpoints of the interval, so both $\partial^{+}\left(\mathbf{R} \mathbf{N}_{\text {med }}^{\text {und }}\right)$ and $\partial^{-}\left(\mathbf{R} \mathbf{N}_{\text {med }}^{\text {und }}\right)$ consist of two null cylinders on $\mathbb{S}^{2}$ (one each for rays going out to $t=\infty$ and $t=-\infty$ ), joined at a spacelike $\mathbb{S}^{2}$. The $t=\infty$ cylinder in $\partial^{-}\left(\mathbf{R N}_{\text {med }}^{\text {und }}\right)$ attaches to the $r=r_{+}$cone in $\partial^{+}\left(\mathbf{R N}_{\text {ext }}^{\text {und }}\right)$.

The inner sector $\mathbf{R} \mathbf{N}_{\text {int }}^{\text {und }}=\mathbb{R} \times\left(0, r_{-}\right) \times \mathbb{S}^{2}$ has $\phi(r)$ behaving in a way that is not covered by Corollary 6.6: $\phi$ is increasing on the entire range, approaching 0 at $r=0$ and infinity at $r=r_{-}$. A full treatment here would require a theorem analogous to Theorem 6.2 covering the case of $a$ being monotonic on $(\alpha, \omega)$ with $a(\alpha)=0$. This is a bit more complicated in that the Riemannian manifold $(\alpha, \omega) \times{ }_{a} K$ does not have all distances realized by geodesics; a typical example is with $(\alpha, \omega)=(0, \infty)$, $a(r)=r$, and $K=\mathbb{S}^{n-2}$, yielding Euclidean space $\mathbb{R}^{n-1}$ minus the origin. But this is not unmanageable, and there appears to be no major obstacle to achieving very similar results (though the case of $a(\alpha)>0$ is considerably messier and the results less clear).

Other than that missing point, how do the proofs change for a monotonic analogue of Theorem 6.2? The first thing to note is that for geodesics heading off to $\alpha$, the limiting radial geodesic $\gamma$ necessarily has $\theta_{\gamma}=\pi$; the analogue of Lemma 6.3 must include inward-radial as a possible (indeed, necessary) conclusion. Lemma 6.4 works the same as before, but use of Lemma 6.5 becomes more complicated: We must instead make use of the note following Lemma 6.5 in an analogue of Part IIa of the Theorem 6.2 proof. Here is how that runs:

We get the same equation for $b_{p}((\bar{\rho}, q))$, in terms of a sequence of numbers $\left\{s_{n}\right\}$ approaching infinity with $\theta_{n}>\pi / 2$, that appears at the end of Part IIa, including the two limit terms involving integrals. Unlike in the original version of Theorem 6.2, in which it was shown that a contradiction arises from the existence 
of the sequence $\left\{s_{n}\right\}$, now we must show why each of these limits is independent of $p$ (the apparent $p$-dependence comes in $\theta_{n}$, the intial radial angle of the minimizing geodesics $\gamma_{n}$ running from $(\bar{\rho}, p)$ to $\left.\left(s_{n}, q\right)\right)$. This is easy for the second limit: We are concerned with $\lim _{n \rightarrow \infty} \int_{\rho_{n}}^{\bar{\rho}}\left(1 / \sqrt{1-\left(\bar{a} \sin \theta_{n} / a(\rho)\right)^{2}}\right) d \rho$, where $\rho_{n}=a^{-1}\left(\bar{a} \sin \theta_{n}\right)$. We just rewrite this as $\lim _{n \rightarrow \infty} \int_{\rho_{n}}^{\bar{\rho}}\left(a(\rho) / \sqrt{a(\rho)^{2}-a\left(\rho_{n}\right)^{2}}\right) d \rho$, with $\left\{\rho_{n}\right\}$ being some sequence approaching $\alpha$, and we see it has no dependence on $p$. (If $\int_{x}^{\bar{\rho}}\left(a(\rho) / \sqrt{a(\rho)^{2}-a(x)^{2}}\right) d \rho$ is continuous in $x$-not obvious, as this is an improroper integral - then the limit is $\bar{\rho}-\alpha$; but we don't need this.)

For the first limit, $\lim _{n \rightarrow \infty}\left(\bar{a}^{2} \sin ^{2} \theta_{n} \int_{\bar{\rho}}^{s_{n}} \frac{d \rho}{a(\rho)^{2} A\left(\theta_{n}\right)\left(A\left(\theta_{n}\right)+1\right)}\right)$, let us denote the integral by $J_{n}$. As $A\left(\theta_{n}\right) \leq 1$, we must have $\lim _{n} J_{n}=\infty$, as we are assuming $\int_{\bar{\rho}}^{\infty} 1 / a(\rho)^{2} d \rho=\infty$. This forces $\left\{\theta_{n}\right\}$ to approach $\pi$ (given that all $\left.\theta_{n}>\pi / 2\right)$, since otherwise we would have $b_{p}((\bar{\rho}, q))=-\infty$, and that is impossible. Thus, all we need to do is show that the sequence $\left\{\bar{a} \sin \theta_{n} J_{n}\right\}$ is bounded above, and we will have $\lim _{n}\left(\bar{a}^{2} \sin ^{2} \theta_{n} J_{n}\right)=0$, independent of $p$. It will suffice to have $\bar{a} \sin \theta_{n} \int_{\bar{\rho}}^{s_{n}} \frac{d \rho}{a(\rho)^{2} A\left(\theta_{n}\right)}$ bounded above; note that this is exactly the same as $L\left(\sigma_{n}\right)$, where $\sigma_{n}$ is the projection of $\gamma_{n}$ to $K$, a pregeodesic from $p$ to $q$. While we don't know that $\sigma_{n}$ is minimizing, we do know, from the note following Lemma 6.5 , that it is the union of at most two minimizing geodesics. It follows that $L\left(\sigma_{n}\right) \leq 2 \operatorname{diam}(K)$, and we are done.

So a monotonic analogue of Corollary 6.6 necessitates we need consider only $\int_{r_{-}-\epsilon}^{r_{-}} 1 / f(r) d r$ and $\int_{0}^{\epsilon} 1 / f(r) d r$; the first is infinite, the second finite. This gives us, for both $\partial^{+}\left(\mathbf{R} \mathbf{N}_{\text {int }}^{\text {und }}\right)$ and $\partial^{-}\left(\mathbf{R} \mathbf{N}_{\text {int }}^{\text {und }}\right)$, a null cone on $\mathbb{S}^{2}$ at $r=r_{-}$conjoined at the vertex $j^{+}$or $j^{-}$to a timelike line at $r=0$ (the central singularity-or one instance of it). The $r=r_{-}$cone in $\partial^{-}\left(\mathbf{R} \mathbf{N}_{\text {int }}^{\text {und }}\right)$ attaches to the $t=\infty$ cylinder in $\partial^{+}\left(\mathbf{R} \mathbf{N}_{\text {med }}^{\text {und }}\right)$. Conventional wisdom is to identify the $r=0$ lines in $\partial^{+}\left(\mathbf{R} \mathbf{N}_{\text {int }}^{\text {und }}\right)$ and $\partial^{-}\left(\mathbf{R N}_{\text {int }}^{\text {und }}\right)$, yielding a timelike line with two endpoints, $j^{+}$and $j^{-}$, for the singularity.

In the maximal extension of $\mathbf{R} \mathbf{N}^{\text {und }}$, the $t=-\infty$ cylinder in $\partial^{-}\left(\mathbf{R} \mathbf{N}_{\text {med }}^{\text {und }}\right)$ attaches to the $r=r_{+}$cone in the future boundary of a $t$-reversed copy $\mathbf{R} \mathbf{N}_{\text {alt }}^{\text {und }}$ of $\mathbf{R N}_{\text {ext }}^{\text {und }}$. We also have the $t=\infty$ cylinder in $\partial^{+}\left(\mathbf{R} \mathbf{N}_{\text {med }}^{\text {und }}\right)$ attaching to the $r=r_{-}$ cone in the past boundary of a $t$-reversed copy $\mathbf{R} \mathbf{N}_{\text {alt-int }}^{\text {und }}$ of $\mathbf{R} \mathbf{N}_{\text {int }}^{\text {und }}$. A typical Penrose diagram also suggests identifying $i^{+}$in $\mathbf{R} \mathbf{N}_{\text {ext }}^{\text {und }}$ with $j_{\text {alt }}^{-}$, the past endpoint (at

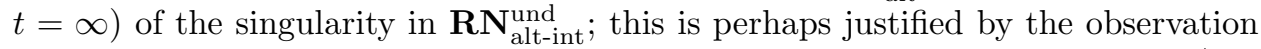
that the elements of the alternate singularity, parametrized by $t$, do approach $i^{+}$as $t$ goes to $\infty$. In similar fashion, $i_{\text {alt }}^{+}$(future timelike infinity, at $t=-\infty$ ) in $\mathbf{R} \mathbf{N}_{\text {alt }}^{\text {und }}$ may be identified with $j^{-}$in $\mathbf{R} \mathbf{N}_{\text {int }}^{\text {und }}$.

Continuing in the maximal extension: A $t$-reversed copy of $\mathbf{R} \mathbf{N}_{\text {med }}^{\text {und }}$ attaches via the two cylinders of its past boundary to the $r=r_{-}$cones in $\partial^{+}\left(\mathbf{R} \mathbf{N}_{\text {int }}^{\text {und }}\right)$ and $\partial^{+}\left(\mathbf{R} \mathbf{N}_{\text {alt-int }}^{\text {und }}\right)$. The future boundary of that copy of $\mathbf{R} \mathbf{N}_{\text {med }}^{\text {und }}$ attaches to the past boundaries of copies of $\mathbf{R} \mathbf{N}_{\text {ext }}^{\text {und }}$ and $\mathbf{R} \mathbf{N}_{\text {alt }}^{\text {und }}$, another copy of $\mathbf{R} \mathbf{N}_{\text {med }}^{\text {und }}$ attaches via its past boundary to the future boundaries of those, and so on, futurewards. Similarly, the $r=r_{+}$cones in $\partial^{-}\left(\mathbf{R} \mathbf{N}_{\text {ext }}^{\text {und }}\right)$ and $\partial^{-}\left(\mathbf{R} \mathbf{N}_{\text {alt }}^{\text {und }}\right)$ attach to the future boundary of another $t$-reversed copy of $\mathbf{R} \mathbf{N}_{\text {med }}^{\text {und }}$, whose past boundary attaches to a continued pastwards extension. 
Thus, the entire causal boundary of $\mathbf{R} \mathbf{N}^{\text {und }}$ consists of the following: a sequence of connected components, each component consisting of a null cone (on $\mathbb{S}^{2}$ ) with vertex $i^{+} / j^{-}$to the future, timelike line leading futurewards from that $i^{+} / j^{-}$to a point $i^{-} / j^{+}$, and a null cone with that $i^{-} / j^{+}$as vertex to the past; and a duplicate of this sequence on the "other side".

Critically charged Reissner-Nordström has $|q|=m$, yielding $f(r)=\left(1-\frac{m}{r}\right)^{2}$. On the exterior portion, $\mathbf{R N}_{\mathrm{ext}}^{\mathrm{crt}}=\mathbb{R} \times(m, \infty) \times \mathbb{S}^{2}$, we have $\phi(r)=\left(r^{2} /(r-m)\right)^{2}$, and we apply Corollary 6.6 as before, with the same result: $\partial^{+}\left(\mathbf{R N}_{\mathrm{ext}}^{\text {crt }}\right)$ and $\partial^{-}\left(\mathbf{R N}_{\mathrm{ext}}^{\text {crt }}\right)$ each consist of two null cones on $\mathbb{S}^{2}$ (one for $r=\infty$ and one for $r=m$ ) conjoined at what might respectively be called $i^{+}$and $i^{-}$. The interior portion, $\mathbf{R} \mathbf{N}_{\text {int }}^{\text {crt }}=$ $\mathbb{R} \times(0, m) \times \mathbb{S}^{2}$, has the same issue as with $\mathbf{R N}_{\text {int }}^{\text {und }}: \phi(r)$ decreases to 0 at $r=0$. Using the same monontonic analogue of Corollary 6.6, we obtain, similarly, a null cone on $\mathbb{S}^{2}$ at $r=m$ conjoined at its vertex $\left(j^{+}\right.$or $\left.j^{-}\right)$to a timelike line at $r=0$, for both $\partial^{+}\left(\mathbf{R N}_{\text {int }}^{\text {crt }}\right)$ and $\partial^{-}\left(\mathbf{R N}_{\text {int }}^{\text {crt }}\right)$. The null cone in $\partial^{-}\left(\mathbf{R} \mathbf{N}_{\text {int }}^{\text {crt }}\right)$ attaches to the $r=m$ cone in $\partial^{+}\left(\mathbf{R N}_{\text {ext }}^{\text {crt }}\right)$. Convention dictates identifying the timelike lines in $\partial^{+}\left(\mathbf{R N}_{\text {int }}^{\text {crt }}\right)$ and $\partial^{-}\left(\mathbf{R N}_{\text {int }}^{\text {crt }}\right)$. In a maximal extension, the $r=m$ cone in $\partial^{+}\left(\mathbf{R N}_{\text {int }}^{\text {crt }}\right)$ attaches to the $r=m$ cone in the past boundary of another copy of $\mathbf{R N}_{\mathrm{ext}}^{\mathrm{crt}}$, which attaches to another copy of $\mathbf{R} \mathbf{N}_{\text {int }}^{\text {crt }}$, and so on furturewards; and similarly pastwards from the $r=m$ cone $\partial^{-}\left(\mathbf{R N}_{\text {ext }}^{\text {crt }}\right)$. A typical Penrose diagram suggests identifying the $i^{-}$of one copy of $\mathbf{R} \mathbf{N}_{\text {ext }}^{\text {crt }}$ with the $i^{+}$of the copy to its immediate past, but justification for this is questionable. However, topology justifies identifying the past endpoint $j^{-}$of the singularity in each copy of $\mathbf{R} \mathbf{N}_{\text {int }}^{\text {crt }}$ with the future endoint $j^{+}$of the singularity in the copy to its immediate past, much as in $\mathbf{R} \mathbf{N}^{\text {und }}$. We obtain, then, for the full causal boundary, the following: in the exterior, a sequence of null cones on $\mathbb{S}^{2}$, alternating with vertex at future or at past (unlcear whether to identify pairs of verticies $i^{-}$and $i^{+}$); and in the interior, a single timelike line (as paired $j^{-}$and $j^{+}$are identified).

Overcharged Reissner Nordström has $|q|>m$, yielding $f(r)>0$ for all $r$ : $\mathbf{R} \mathbf{N}^{\text {ovr }}=\mathbb{R} \times(0, \infty) \times \mathbb{S}^{2}$. We have $\phi(r)$ decreasing to 0 at $r=0$ and eventually increasing to infinity at $r=\infty$. We have $\int_{0}^{m} 1 / f(r) d r<\infty$ and $\int_{m}^{\infty} 1 / f(r) d r=\infty$. A monotonic analogue of Corollary 6.8 tells us $\partial^{+}\left(\mathbf{R} \mathbf{N}^{\text {ovr }}\right)$ and $\partial^{-}\left(\mathbf{R} \mathbf{N}^{\text {ovr }}\right)$ are each a null cone on $\mathbb{S}^{2}$ at $r=\infty$ joined, at the vertex $i^{+}$or $i^{-}$, to a timelike line at $r=0$; conventionally, the timelike lines are identified yielding a single timelike line from $i^{-}$to $i^{+}$for the singularity. Thus, the entire entire causal boundary consists of a null cone (on $\mathbb{S}^{2}$ ) with vertex $i^{-}$to the past, a timelike line leading futwards from $i^{-}$to a point $i^{+}$, and a null cone with that $i^{+}$as vertex to the future.

What is perhaps most noteworthy here is that the singularity in $\mathbf{R N}$ is onedimensional, as opposed to the $\mathbb{R} \times \mathbb{S}^{2}$ structure of the singuarity in Sch (or SdS or SAdS). For the spacelike singularities (of Schwarzschild character), this is a topologically universal result, as detailed in [H2]; for the timelike Reissner-Nordström singularity, it is at least causally universal, as detailed in [H1]. 


\section{REFERENCES}

[BGS] W. Ballman, M. Gromov, and V. Schroeder, Manifolds of Non-positive Curvature, Birkhäuser, 1985.

[BS] R. Budic and R. K. Sachs, Causal boundaries for general relativistic space-times, J. Math. Phys. 15 (1974), 1302-1309.

[F] J. L. Flores, The causal boundary of spacetimes revisited, preprint.

[GH] D. Garfinkle and S. G. Harris, Ricci fall-off in static and stationary, globally hyperbolic, non-singular spacetimes, Class. Quantum Grav. 14 (1997), 139-151.

[GKP] R. P. Geroch, E. H. Kronheimer, and R. Penrose, Ideal points in space-time, Proc. Roy. Soc. Lond. A 327 (1972), 545-567.

[GiH] G. W. Gibbons and S. W. Hawking, Cosmological event horizons, thermodynamics, and particle creation, Phys. Rev. D 15 (1977), 2738-51.

[H1] S. G. Harris, Universality of the future chronological boundary, J Math Phys 39 (1998), $5427-5445$.

[H2] Topology of the future chronological boundary: universality for spacelike boundaries, Class. Quantum Grav. 17 (2000), 551-603.

[H3] Causal boundary for standard static spacetimes, Nonlin. Anal. 47 (2001), 29712981, Special Edition: Proceedings of the Third World Congress in Nonlinear Analysis.

[H4] Discrete group actions on spacetimes: causality conditions and the causal boundary, Class. Quantum Grav. 21 (2004), 1209-1236, online at gr-qc/0310071.

[HE] S. W. Hawking and G. F. R. Ellis, The Large Scale Structure of Space-Time, Cambridge University, Cambridge, 1973.

[M] S. Mac Lane, Categories for the Working Mathematician, Springer-Verlag, New York, 1971.

[MR] D. Marolf and S. F. Ross, A new recipe for causal completions, Class, Quantum Grav. 20 (2003), 4085-4117, online at gr-qc/0303025.

[R] W. Rindler, Relativity: Special, General and Cosmological, Oxford University Press, Oxford, 2001.

[S] L. B. Szabados, Causal boundary for strongly causal spacetimes, Class. Quantum Grav. 5 (1988), 121-134; Causal boundary for strongly causal spacetimes: II, Class. Quantum Grav. 6 (1989), 77-91.

Department of Mathematics, Saint Louis University, St. Louis, MO 63103, USA

Current address: Departamento de Álgebra, Geometría, y Topología, Facultad de Ciencias, Universidad de Málaga, 29071 Málaga, Spain

E-mail address: floresj@agt.cie.uma.es

Department of Mathematics, Saint Louis University, St. Louis, MO 63103, USA and Department of Mathematics, University of Missouri-Columbia, Columbia, MO 65221 , USA

Current address: Department of Mathematics, Saint Louis University, St. Louis, MO 63103, USA

E-mail address: harrissg@slu.edu 